\title{
System Performance and Cost Sensitivity Comparisons of Stretched Membrane Heliostat Reflectors with Current Generation Glass/Metal Concepts
}

\author{
L. M. Murphy \\ J. V. Anderson \\ W. Short \\ T. Wendelin
}

December 1985

Prepared under Task No. 5111.31

FTP No. 510

Solar Energy Research Institute

A Division of Midwest Research Institute

1617 Cole Boulevard

Golden, Colorado 80401

Prepared for the

U.S. Department of Energy

Contract No. DE-AC02-83CH10093 


\title{
NOTICE
}

This report was prepared as an account of work sponsored by the United States Government. Neither the United States nor the United States Department of Energy, nor any of their employees, nor any of their contractors, subcontractors, or their employees, makes any warranty, expressed or implied, or assumes any legal liability or responsibility for the accuracy, completeness or usefulness of any information, apparatus, product or process disclosed, or represents that its use would not infringe privately owned rights.

\section{Printed in the United States of America \\ Available from: \\ National Technical Information Service \\ U.S. Department of Commerce \\ 5285 Port Royal Road \\ Springfield, VA 22161}

\author{
Price: Microfiche A01 \\ Printed Copy A05
}

Codes are used for pricing all publications. The code is determined by the number of pages in the publication. intormation pertaining 10 the pricing codes can be found in the current issue of the following publications, which are generally available in most libraries: Energy Research Abstracts, (ERA); Government Reports Announcoments and Index (GRA and 1): Scientific and Technical Abstract Reports (STAR); and publication, NTIS-PR-360 avallable from NTIS at the above address. 
PREFACE

The research and development described in this document was conducted within the U.S. Department of Energy's Solar Thermal Technology Program. The goal of this program is to advance the engineering and scientific understanding of solar thermal technology and to establish the technology base from which private industry can develop solar thermal power production options for introduction into the competitive energy market.

Solar thermal technology concentrates the solar flux using tracking mirrors or lenses onto a receiver where the solar energy is absorbed as heat and converted into electricity or incorporated into products as process heat. The two primary solar thermal technologies, central receivers and distributed receivers, employ various point and line-focus optics to concentrate gunlight. Current central receiver systems use fields of heliostats (two-axis tracking mirrors) to focus the sun's radiant energy onto a single, towermounted receiver. Point focus concentrators up to 17 meters in diameter track the sun in two axes and use parabolic dish mirrors or Fresnel lenses to focus radiant energy onto a receiver. Troughs and bowls are line-focus tracking reflectors that concentrate sunlight onto receiver tubes along their focal lines. Concentrating collector modules can be used alone or in a multimodule system. The concentrated radiant energy absorbed by the solar thermal receiver is transported to the conversion process by a circulating working fluid. Receiver temperatures range from $100^{\circ} \mathrm{C}$ in $10 \mathrm{w}$-temperature troughs to over $1500^{\circ} \mathrm{C}$ in dish and central receiver systems.

The Solar Thermal Technology Program is directing efforts to advance and improve each system concept through solar thermal materials, components, and subsystems research and development and by testing and evaluation. These efforts are carried out with the technical direction of DOE and its network of field laboratories that works with private industry. Together they have established a comprehensive, goal-directed program to improve performance and provide technically proven options for eventual incorporation into the Nation's energy supply.

To successfully contribute to an adequate energy supply at reasonable cost, solar thermal energy must be economically competitive with a variety of other energy sources. The Solar Thermal Program has developed components and system-level performance targets as quantitative program goals. These targets are used in planning research and development activities, measuring progress, assessing alternative technology options, and developing optimal components. These targets will be pursued vigorously to ensure a successful program.

This report presents work supported by the Division of solar Thermal Technology of the U.S. Department of Energy (DOE) as part of the Solar Energy Research Institute research effort on innovative concentrators. Research on stretched membrane heliostat modules has been emphasized as part of the innovative concentrator effort for some time because of their potential cost/performance benefits over the current technology for heliostats. However, the potential of stretched membrane heliostats from a systems perspective over a wide range of design and operational parameters has not been studied until this time. The purpose of this report is to document and 
present the findings of a study comparing stretched membrane and glass/metal heliostats from a systems perspective. In this study, we have investigated the sensitivity of the relative performance and cost of fields of heliostats to a fairly large number of parameter variations, including system size and delivery temperature, along with heliostat module size, reflective surface specularity, hemispherical reflectance, and macroscopic surface quality for both focused and unfocused stretched membrane modules.

The authorg would like to thank both Martin Scheve and Frank Wilkins of the U.S. Department of Energy for their support in this effort. The authors would also like to note that this work builds upon an extensive information base on the optical performance of solar concentrators that has been developed for the DOE Solar Thermal Program by the Sandia National Laboratories.

Approved for

SOLAR ENERGY RESEARCH INSTITUTE

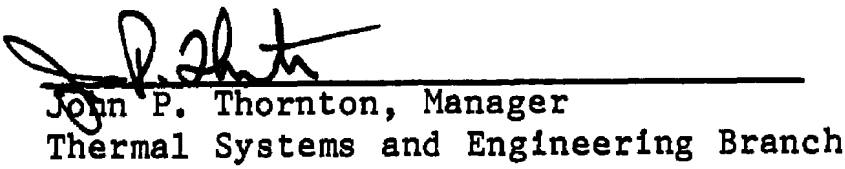

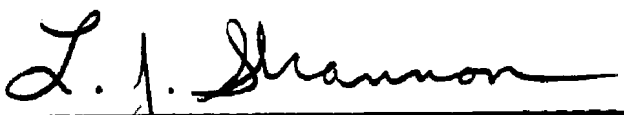

L. J. Spannon, Director

Solar Yeat Research Division 


\section{SUMMARY}

In this report we compare stretched membrane heliostats with state-of-the-art glass/metal heliostats from a systems perspective. We have investigated the sensitivity of the relative performance and cost of fields of heliostats to a large number of parameter variations, including system size, delivery temperature, heliostat module size, surface specularity, hemispherical reflectance, and macroscopic surface quality for both focused and unfocused stretched membrane modules.

Effort in this project has been directed in two major areas:

- Assessing the performance of individual modules based on the findings of current structural analyses for stretched membrane modules, including the review of existing assumptions and practices for analyzing heliostats

- Conducting systems sensitivity studies of fields of heliostats on which the parameter variations are performed.

The performance comparisons are based on the annual delivered energy. The system designs are optimized for performance only. Cost sensitivities are based on assumed costs for these performance optimized systems.

A major feature of this study is that it brings together into a unified presentation a large number of results and definitions from systems, structural, and optical materials investigations conducted for DOE by Sandia National Laboratories (SNLL) and the Solar Energy Research Institute (SERI).

\section{GROUAD RULES AND MAJOR ASSUMPTIONS}

Some of the ground rules and major assumptions used in this study are 1 isted below.

\section{Heliostats}

- 100- $\mathrm{m}^{2}$ glass/metal heliostats were used as the baseline standard of comparison as in DeLaquil and Anderson [11]. Each heliostat has 12 mirror panels in a $2 \times 6 \mathrm{ft}$ array focused in 2 directions and canted on-axis.

- Both focused and unfocused stretched membrane heliostats using the double membrane construction were studied. They were sized at $25 \mathrm{~m}^{2}, 50 \mathrm{~m}^{2}$, and $100 \mathrm{~m}^{2}$.

- The focused stretched membrane heliostats were assumed to use the vacuum/pressure active control of the reflector surface proposed by SNLL.

- Al1 deformations in the unfocused stretched membrane helioatats were measured relative to a perfectly flat reference plane.

- We have utilized standard documented approaches in considering reflector surface error effects. Specifically, in the analysis and the discussions we assumed that specular reflectivity can be represented as the product 
of two independent functions; i.e., the total hemispherical reflectivity times a geometric function of the cone angle containing the scattered reflective beam. We considered independent variations in both the hemispherical reflectivity and the variance of the cone angle about the specvlar directions. In addition, macroscopic surface quality effects caused by wind-, weight-, and manufacturing-induced errors are considered as reflected-beam broadening effects along with the microscopic specularity variations about the specular direction.

\section{Balance of system}

- Only single cavity, open aperture receivers coupled to north fields were considered.

- The study investigated IPH systems with delivery temperatures of $450^{\circ}$, $750^{\circ}$, and $1050^{\circ} \mathrm{C}$ for plant sizes of $75 \mathrm{MW}_{t h}$ and $450 \mathrm{MW}_{t h}$ (field sizes of approximately $100,000 \mathrm{~m}^{2}$ and $700,000 \mathrm{~m}^{2}$ ).

- No storage was considered for any of the cases.

\section{PINDIMGS ARD CONCLUSIONS}

Based on the ground rules and assumptions (as well as the other limitations listed in the body of the report), the major findings of this study are 1 isted below, corresponding to focused stretched membrane heliostat modules, unfocused modules, and general considerations for both focused and unfocused stretched membrane heliostats. The rationale for a number of the conclusions is demonstrated in Figure $\mathrm{S}-1$, which shows the annual performance of various systems as a function of temperature. Since all of the performance values are normalized to that of a system using glasa/metal heliostats, direct comparisons to the state-of-the-art baseline can be made.

\section{Bocused Stretched Membrane Heliostats}

- Overall, focused stretched membrane heliostats appear to have the potential to perform at levels that are quite close to glass/metal heliostats. However, to achieve this parity in performance it is important that the optical quality--in particular, the reflectivity and the macroscopic surface errors introduced in manufacturing--be comparable to that of the glass/metal heliostats.

- The performance of focused stretched membrane heliostats appears to have the same sensitivity to variations in optical quality parameters as the performance of glass/metal heliostats.

- The performance of the focused stretched membrane heliostats is nearly independent of heliostat size for the plant sizes studied.

- The round shape of the stretched membrane heliostats gives them a slight $(2 \%-5 \%)$ advantage over the square glass/metal heliostats, since it simultaneously allows tighter field packing and lower shading and blocking losses. This can be seen on Figure S-1, where the normalized performance 


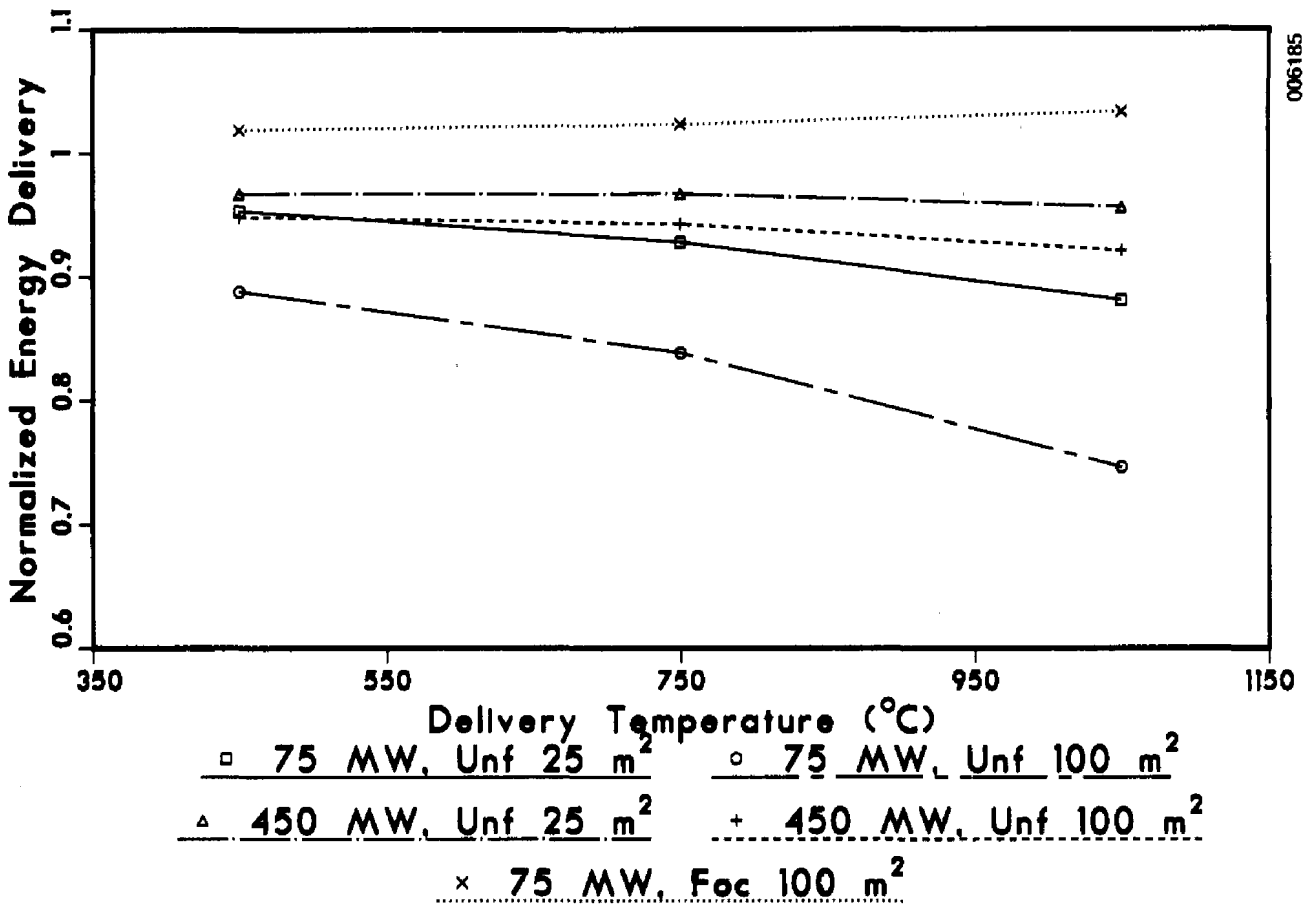

Rigure s-1. Annual Energy Delivery for Several Stretched Membrane Heliogtat Systems Mormalized by the Energy Delivered by a Glass/Metal Heliostat System as a Punction of the Delivery Temperature. The results are for both $75 \mathrm{MW}$ - and 450-MW plants, using two sizes of unfocused heliostats and a single size of focused heliostats.

of the focused system lies several percentage points above 1.0 . This advantage might be reduced by clipping the corners of the glass/metal heliostats to approximate a circle.

\section{Unfocused Stretched Membrane Heliostats}

- A reasonable upper bound has been established for the surface errors corresponding to the axisymmetric deformations of the reflector membrane caused by wind and weight loading. It has also been established that these errors are inversely proportional to the design tension levels and directly proportional to the diameter of the module, and are thus controllable by appropriate design.

- Heliostat diameter is a very important parameter when considering unfocused stretched membrane heliostats because:

- the size of the image at the receiver is strongly dependent on the heliostat size for the unfocused modules

- it appears that it will be possible to achieve lower levels of surface normal errors with smaller diameter heliostats.

This conclusion is also supported by Figure s-1. The curves for the $100-\mathrm{m}^{2}$ unfocused stretched membrane heliostats always lie below those for the $25-\mathrm{m}^{2}$ units at the same plant size. 


\section{General Considerations}

- In general, the performance sensitivity of central receiver systems to poorer optical quality in the heliostats tends to increase with higher temperatures and smaller plant sizes. This conclusion is borne out by the curves in Figure $s-1$.

- Focusing seems quite desirable if a single heliostat design is to have the largest range of applicability. Based on the performance analyses, focusing is especially beneficial for small plant sizes and high temperatures. However, as the plant size increases and/or the temperature decreases, the benefit of focusing rapidly diminishes.

- For the baseline assumptions we found that the sensitivity of system performance to variations in specularity errors was much less than that corresponding to variations in either hemispherical reflectivity or surface normal errors. For the range of systems studied, the specularity halfcone angle that includes $90 \%$ of the reflected energy can be as large as about $6 \mathrm{mrad}$ (from a baseline of $1 \mathrm{mrad}$ ) while producing a less than $5 \%$ decrease in annual system performance. This insensitivity to specularity errors is due primarily to the dampening effect provided by the other error sources.

- Underestimation of the heliostat optical surface error used in the optimization of the system will lead to system designs that reduce delivered energy levels significantly below those that might be produced by a system designed at the actual or higher optical error levels. 
TABLE OF CONTENTS

1.0 Introduction...................................... 1

2.0 Individual Module Performance............................ 3

2.1 Optical Quality and Sources of Error.................... 3

2.2 Macroscopic Surface Accuracy in Wind and Weight Environments..... 5

2.3 Assumptions for Individual Modules...................... 8

3.0 Systems Studies on the Focused Stretched Membrane Module........... 11

3.1 Assumptions and Ground Rules......................... 11

3.2 Results for Focused Membrane Modules...................... 12

3.3 Impact of Optical Error Uncertainties.................... 22

4.0 Systems Analysis of Unfocused Stretched Membrane Heliostats......... 25

4.1 Assumptions....................................... 25

4.2 Results for Unfocused Stretched Membrane Systems............. 26

5.0 Conclusions....................................... 33

6.0 References........................................ 35

Appendix A Differences between Probabilistic and Deterministic

Surface Errors................................... 37

Appendix B Anticipated Loading and the Resulting Surface Errors for Individual stretched Membrane Modules................... 50

Appendix C Performance Optimized Central Receiver Design Process........ 62

Appendix D Performance Optimization versus Cost/Performance Optimization.................................... 69

Appendix E Specularity and Cone Size Relations.................... 71

Appendix $\mathbf{F}$ Receiver Configuration............................ 74 


\section{LIST OR FIGURES}

S-1 Annual Energy Delivery for Several Stretched Membrane Heliostat Sygtems Normalized by the Energy Delivered by a Glass/Metal Heliostat System as a Function of the Delivery Temperature................. vii

2-1 Definition of the Specularity Cone in Terms of the Angle about the Nominal Reflected Ray.................................

2-2 Specularity Errors vs. Surface Normal Errors for a Given Level of Total Surface Error Showing the Tradeoff between Specularity and Surface Errors.......................

3-1 Annual Delivered Energy per $\mathrm{m}^{2}$ of Heljostat for Focused Stretched Membrane Heliostats and Current $100-\mathrm{m}^{2}$ Glass/Metal Heliostats at Two Plant sizes as a Function of Temperature..................

3-2 Annual Energy Delivered as a Function of Focused Stretched Membrane Heliostat Area..................................

3-3 Annual Energy Delivered by Stretched Membrane Heliostat Systems with Various Levels of Surface Error, Normalized by the Annual Energy Delivered by State-of-the-Art Glass/Metal Heliostat System for a $75-M W_{t h}$ Plant...............................

3-4 Annual Energy Delivered by Stretched Membrane Heliostat Systems with Various Levels of Surface Error, Normalized by the Annual Energy Delivered by State-of-the-Art Glass/Metal Heliostat System for a $450-\mathrm{MW}_{\mathrm{th}}$ Plant............................ 16

3-5 Levelized Annual Energy Cost as a Function of Surface Error for the 75-MW Plant at Two Delivery Temperatures and Two Heliostat Costs ....

3-6 Levelized Annual Energy Cost as a Function of Surface Error for the 450-MW Plant at Two Delivery Temperatures and Two Heliostat

Costs.................................................

3-7 Normalized Annual Energy Delivery from Focused Stretched Membrane Heliostats as a Function of the Standard Deviation of the Specularity Errors for a 75-MW Plant at Three Delivery

Temperatures......................................

3-8 Levelized Annual Energy Cost as a Function of the Standard Deviation of the Specularity Errors for the 75-MW Plant at Two Delivery Temperatures and Two Heliostat Costs............... 
3-9 Allowable Heliostat Cost as a Function of the Specularity Half Cone Angle for Several Leyels of Levelized Energy Cost for a 75-MW Plant Using 100-m $\mathrm{m}^{2}$ Focused Stretched Membrane Heliostats at a Delivery Temperature of $750^{\circ} \mathrm{C} \ldots \ldots \ldots \ldots \ldots \ldots \ldots \ldots \ldots \ldots \ldots \ldots$

3-10 Normalized Annual Energy Delivery from Focused Stretched Membrane Heliostats as a Function of the Hemispherical Reflectivity

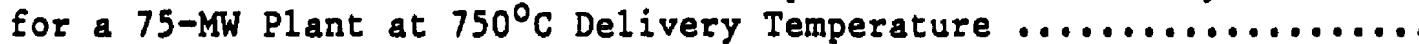

3-11 Levelized Annual Energy Cost as a Eunction of the Hemispherical Reflectivity, for a 75-W Plant with a Delivery Temperature

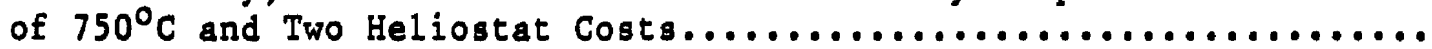

3-12 A Comparison of the Relative Effects of Several Optical Parameters on the Annual Energy Delivery for a 75-MW Plant Using Focused Stretched Membrane Heliostats..........................

3-13 A Comparison of the Relative Effects of Several Optical Parameters on the Levelized Energy Cost for a 75-MW Plant Using $\$ 50 / \mathrm{m}^{2}$ Eocused Stretched Membrane Heliostats..............

3-14 Normalized Annual Delivered Energy vs. $\sigma_{\text {opt }}$ Corresponding to Three Optimization Procedures.............................

4-1 Comparison of the Baseline Cases for Glass/Metal and Focused and Unfocused Stretched Membrane Heliostats......................

4-2 Ratio of Annual Energy Delivery for the Unfocused Stretched Membrane Heliostats to That for the Focused Stretched Membrane Heliostats as a Function of Delivery Temperature for 3 Heliostat Sizes and a 75-MW Plant...........................

4-3 Ratio of Annual Energy Delivery for the Unfocused Stretched Membrane Helioatats to That for the Focused Stretched Membrane Heliostats as a Function of Delivery Temperature for 3 Heliostat sizes and a $450-M W$ Plant..........................

4-4 Allowable Cost of Focusing as a Function of Delivery Temperature for a 75-MW Plant at Several Heliostat Sizes and Total Surface Errors...............................................

4-5 Allowable Cost of Focusing as a Function of Delivery Temperature for a 450-MW Plant at Several Heliostat Sizes and Total Surface Errors..............................................

4-6 Effect of Surface Errors on the Annual Energy Delivery for Several Sizes of Unfocused Stretched Membrane Heliostats in a 75-MW Plant at $750^{\circ} \mathrm{C}$ Delivery Temperature....................... 
4-7 Effect of Surface Errors on the Annual Energy Delivery for Several Sizes of Unfocused Stretched Membrane Heliostats in a $450-\mathrm{MW}$ Plant at $750^{\circ} \mathrm{C}$ Delivery Temperature..................... 31

A-1 Definition of the Surface Normal Error....................... 38

A-2 Comparison of the Deterministic Surface Normal Error Distribution and a Circular Normally Distributed Approximation for $\mathrm{n}=3$ and $\mathrm{b}=0 \ldots \ldots \ldots \ldots \ldots \ldots \ldots \ldots \ldots \ldots \ldots \ldots \ldots \ldots \ldots \ldots \ldots \ldots$

A-3 Comparison of the Density and Distribution Functions for $\mathrm{n}=2,3$, and 4 , when $b=0, \ldots \ldots \ldots \ldots \ldots \ldots \ldots \ldots \ldots \ldots \ldots \ldots \ldots \ldots$

A-4 Comparison of the Deterministic Surface Normal Error Distribution and a Circular Normally Distributed Approximation for $c=0 \ldots \ldots \ldots .44$

B-1 Assumed Uniform Pressure Loading on the Double Membrane Collector...........................................

B-2 Effective Expected Module Load Corresponding to Axisymmetric Membrane Deformation as a Function of Zenith Angle for

Several Membrane Designs.................................

B-3 Effective RMS Surface Error as a Function of $\bar{P}_{A X}$ for

Several Model Designs..................................

B-4 Average Load Corresponding to Asymmetric Loading for Several

Model Designs.........................................

B-5 Effective Expected Module Area Load Corresponding to Asymmetric Deformation as a Function of Zenith Angle for Several Module Weights...................................

B-6 Example of Module Construction............................

B-7 Torsional and Flexural Rigidity as a Function of Frame Half Height..............................................

B-8 Maximum Frame Deflection as a Function of Frame Half Height for Several Construction Types................................60

B-9 RMS (Surface Averaged) Error as a Function of Frame Half Height..... 60

B-10 RMS (Surface Averaged) Error as a Function of Total Module Weight per Unit of Reflector Area...............................61

C-1 Flowchart of Design Procedure........................... 62 


\section{LIST OF FIGURES (Concluded)}

$\underline{\text { Page }}$

C-2 Variation of System Performance with Tower Height...............64

C-3 (a) Expanded Layout and (b) Isometric View of the Baseline Receiver Cavity Configuration.................................. 66

E-1 Cone Containing Scattered Beam about the Specular Direction........ 71

E-2 Eraction of Reflected Energy in a Given Cone Size.............. 73

F-1 Isometric View of Alternate Receiver Cavity Configuration Allowing Larger Aperture Sizes.......................... 75

F-2 Fractional Change in Delivered Energy as a Result of the Longer Aperture Sizes Possible, with the Reconfigured Cavity

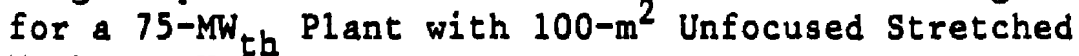
Membrane Hellostats................................... 76 
SEP *

$T R-2694$

LIST OR TABLES

$\underline{\text { Page }}$

s-1 Baseline Surface Quality Assumptions.......................

3-1 Levelized Energy Cost Assumptions for Focused Stretched Membrane and Glass/Metal Heliostat................................... 12

3-2 Error Parameters of Figures $3-3$ and $3-4 \ldots \ldots \ldots \ldots \ldots \ldots \ldots \ldots \ldots \ldots$

A-1 Errors Introduced by the Assumption of a Normally Distributed

Beam Width when $b=0$

43

A-2 Root-Mean-Square Error Results......................... 47

A-3 Error Introduced in a Single Dimension by a Normal Approximation when $b=0, n=2 \ldots \ldots \ldots \ldots \ldots \ldots \ldots \ldots \ldots \ldots \ldots \ldots \ldots \ldots \ldots \ldots \ldots \ldots \ldots$

B-1 Selected Wind Velocity Characteristics for Several Locations......... 54

xiv 


\section{SECTION 1.0}

\section{INTRODUCTION}

This report compares, from a systems perspective, the cost/performance potential for stretched membrane heliostat concepts relative to that of mature glass/metal heliostat concepts. In addition to providing this comparison, the system study perspective is required to improve the accuracy of previous estimates of the annual energy delivered by a field of such concentrators. This report also documents the sensitivity of stretched membrane heliostat field performance to variations in numerous system parameters such as application temperature, field size, heliostat module size, reflector surface quality, and focusing capability.

This work is important in that it puts the glass/metal heliostat and the stretched membrane heliostat on a comparable basis so that research decisions and priorities can be set for the development of the stretched membrane concept. This work will also help us to define and quantify the benefits of specific research issues and to identify the critical research and development efforts required on the stretched membrane concept.

The need for heliostats with dramatically improved cost and performance is discussed by Murphy [1] and is supported by the value-based cost goal analysis developed by a joint industry and DOE cost goal committee [2]. The studies show that for initial cost competitiveness in regions with relatively high annual insolation, mass-produced heliostats that have performance levels close to those of the current glass/metal heliostats and cost about $\$ 100 / \mathrm{m}^{2}$ installed are needed. For more widespread competitiveness with a broad range of conventional fuels, the required cost is about $\$ 50-\$ 60 / \mathrm{m}^{2}$ installed. Further, the need for and use of low-cost heliostat technology is not limited to solar thermal power applications but potentially may benefit large-scale photovoltaic applications as well as daylight applications, where large, lowcost, two-axis tracking platforms can greatly enhance the cost/performance of such systems.

When mass produced, the glass/metal heliostats may reach the $\$ 100 / \mathrm{m}^{2}$ level, but another approach may be required to reach the $\$ 50-\$ 60 / \mathrm{m}^{2}$ level. Because of the promise indicated in earlier DOE studies $[1,3]$, research on the stretched membrane heliostat concept has been under way for some time and has recently become a focus of DOE development. In this concept, a high-strength structural membrane coated with a highly reflective surface is stretched uniformly on a structural frame (typically a lightweight, hollow, toroidal structure). The stretched membrane concept is a structurally efficient method of attaining and supporting a large, optically accurate surface. By supporting the optical surface with a membrane structure, more of the material can be stressed to higher average levels, resulting in both lighter weight and lower cost structures. Further, the stretched membrane can provide a reflective surface that tends to smooth out and attenuate surface irregularities emanating at the supports as well as other surface perturbations inside the periphery of the supports. This concept also appears to be especially suitable for the use of polymer reflectors and polymer structural membranes, which may further reduce weight and cost and improve handing at the factory, in the field, and in transport. 
Following a presentation and discussion on the anticipated accuracy bounds for individual stretched membrane heliostat modules, the system evaluations are described. 
SECTION 2.0

\section{INDIVIDUAL MODULE PERFORMANCE}

In this section, we provide a brief overview of performance predictions for individual modules based on analysis findings from a number of previous studies. The structural analysis performed to date addresses the likely effects of wind and weight-loading environments on the structural/optical performance of simple stretched membrane modules as well as some of the impacts of initial imperfections. Much more detail on the supporting analysis of individual modules can be found in Murphy and Sallis [4], Murphy [5], and Murphy et al. [6]. We provide here only a summary of these findings and the associated impacts relating to the macroscopic optical surface accuracy of individual modules.

A significant, though not complete, knowledge base has been developed to predict the structural deformation/response of stretched membrane modules under specified loading conditions $[4,5,6]$. It is felt that reasonably accurate predictions of the optical accuracy for individual modules can be made for specific configurations and loading on the modules using this knowledge base. It should be noted however, that an optimum configuration has not been defined and that, as with the glass/metal heliostats, the loading environment on concentrators is never fully deterministic since the wind flow approaching the field typically can be described only in a statistical manner. The nature of the wind environment within the field is not well understood because of the extremely complex turbulent flow that exists there. However, performance estimates of individual modules can be made by defining some average anticipated loading and then determining the response of the stretched membrane modules to those defined conditions.

Though the major structural response mechanisms have been studied, numerous issues such as module support effects, dynamic effect details, and the effect of anticipated manufacturing tolerances have not been investigated thoroughly. With respect to manufacturing tolerances and initial imperfections, previous analyses have addressed the amplification effects corresponding to initial imperfections [5] but not the levels that can be anticipated in an actual manufacturing environment; this issue is currently being addressed through development contracts being managed by the Sandia National Laboratories at Livermore (SNLL). This same development activity should also lead to a better definition of the ultimate costs that might be attained with the stretched membrane concept. In addition, various static and dynamic structural response issues are being experimentally investigated. These ongoing activities should help better define the exact performance of specific designs in the future.

\subsection{OPTICAL QUALITY AND SOURCES OF ERROR}

The optical quality of a reflector can be defined as the ability of the reflector to redirect incident solar beam radiation in a specular manner to a given specified target area. A number of error sources that limit that ability are associated with the concentrator. Major sources of error include the macroscopic surface waviness effects, specularity effects, tracking errors, 
pointing errors, loss of hemispherical reflectivity, and the finite sun size.* All of the above effects, except for loss of hemispherical reflectivity, can be viewed as broadening or scattering effects of the redirected radiation.

Macroscopic surface waviness gives rise to variations in the direction of the surface normal and can be induced by a large number of phenomena. These include the inherent variations in the smoothness of the material surface, structural deformations caused by wind and weight loading, and variations in the thickness of one or more component layers (such as the adhesives) that typically make up the reflective surface.

The macroscopic surface waviness is impacted in numerous ways by the manufacturing process. For instance, the accuracy with which the component parts, such as the frame and membrane, can be fabricated before their assembly is completed is extremely important in determining the optical quality of the assembled product (e.g., the planarity of the frame before attaching the membrane to it, or the flatness of the strips that compose the elements of the membrane before being seamed to form the final large sheet). Moreover, the uniformity of the thickness of the material stock in the basic membrane material can also have a significant impact, as can anisotropic material properties in the sheet material. In addition, the method of assembly and materials used in joining the component elements into the final product are of concern. For instance, the way the frame is compressed and constrained during the attachment of the membrane to it can affect the level to which initial imperfections are amplified by the membrane tension.

As noted above, these questions are now being addressed in development contracts managed by SNLL. We are encouraged initially at the prospects for good macroscopic optical quality, since the current contractors are optimistic about their ability to manufacture high quality optical surfaces. We are also encouraged that the simple nature of the reflector, the low number of parts to be assembled, and the inherently forgiving nature of the structure will lead to good macroscopic surface quality for the manufactured module.

Microscopic surface specularity effects cause scattering such that an incoming beam is not reflected in a single ray but rather as a cone of rays whose size depends on the microscopic surface qualities. In good reflectors most of the reflected energy is confined within a very small cone (usually a few milliradians in width) about the nominal reflected ray (specular direction). However, even for fairly good reflectors, a small amount of energy can be scattered at very large angles, regulting in a $108 s$ of energy at the receiver. Loss of hemispherical reflectance is caused by absorption of the incoming rays at the surface so that a finite portion of the incoming beams is not specularly reflected or scattered but rather is absorbed.

*These issues have been discussed extensively in numerous prior studies $[7,8,9]$. Another common term often used in the literature is specular reflectivity, which can be defined, as in Pettit et al. [7], as the product of total hemispherical reflectance (a constant) times a geometric distribution function that gives the percent of the total reflected energy within a given cone angle about the specular directions (see Appendix E). 
Tracking and pointing errors arise because of limitations in positioning the reflector in exactly the desired direction; these errors represent limitations to the collection system, but are not fundamentally surface quality limitations. Similarly, the finite sun image size always causes the reflected image of the sun to be finite even if the reflector were otherwise perfect, and represents a fundamental limitation on the concentration of any concentrator.

In this study, we look at macroscopic variations in surface waviness, specularity effects, and hemispherical reflectivity (solar averaged reflectivity) effects. Since the anticipated errors caused by surface waviness effects are a function of the individual concentrator type and design, we examine them both in terms of the performance of individual heliostat modules (Section 3.0 ) and from a systems perspective. However, since specularity and hemispherical reflectivity effects are not primarily dependent on the concentrator concept, but rather on the microscopic reflective surface material quality, we look at these issues only in the systems analysis sections (Sections 4.0 and 5.0 ). In the systems analysis we look at variations from the baseline values assumed for glass/metal heliostats. In all our analyses all other error sources, including those not associated with the concentrator, such as tower sway (a moving target), tracking errors and foundation motion are assumed to be the same as for the glass/metal heliostats.

\subsection{MACROSCOPIC SURFACE ACCURACY IN WIND AND WEIGHT ENVIRONMENTS}

We describe the optical accuracy of stretched membrane modules in terms of the macroscopic surface quality of the stretched membrane surface. A measure of this macroscopic surface quality is given by the deviation $(\phi)$ of the surface normals from their desired direction. A convenient definition for our purposes is the surface-averaged root mean square (RMS) surface slope error given by

$$
\mathrm{RMS}=\left\langle\bar{\phi}^{2}\right\rangle 1 / 2=\left[\frac{\int \phi^{2} \mathrm{dA}}{\mathrm{dA}}\right]^{1 / 2},
$$

where $\mathrm{dA}$ is the differential surface area.

This measure was chosen for two reasons: it is a convenient way to describe, in an average sense, the macroscopic surface quality of the entire collector surface; and it can be related to the probabilistic reflected beam scattering error measures typically used in analysis tools such as DELSOL2 [10]. In Appendix A we compare the normally distributed error models of tools such as DELSOL2 [10] with the deterministic distribution of errors over the stretched membrane surface. The comparison is made by assuming that the RMS surface slope error modeled by a circular normal probability distribution is equal to the RMS surface slope error as calculated from the deterministic approach. Appendix A shows that the circular normal distribution approximation is adequate for our purposes since we are trying only to bound the anticipated surface errors. The adequacy of this approach is reinforced by the fact that the wind and weight load induced surface errors are probablistic with time and are combined with numerous other errors that are also assumed to be circular normally distributed. For such situations, the Central limit Theorem states that the distribution of a random variable equal to the sum of independent random variables (not all necessarily normally distributed) approaches a normal distribution. 
The cumulative distribution function, $F(\zeta)$ (the probability that the optical scattering angle is less than 5 ), of the circular normal probability distribution approximation is

$$
F(\zeta)=\int_{0}^{2 \pi} \int_{0}^{\zeta} \frac{1}{2 \pi \sigma_{o p t}^{2}} \exp -\left(\frac{\phi^{2}}{2 \sigma_{o p t}^{2}}\right) \phi d \phi d \theta,
$$

where $\sigma_{\text {opt }}$ is the standard deviation of the optical scattering angle as measured ${ }^{\text {in }}$ one dimension ( $i . e$, , as measured along a line intersecting and orthogonal to the normal from a perfect heliostat surface). The circular normal distribution implicitly assumes that the scattering angle in this one dimension is normally distributed with mean zero and standard deviation $\sigma_{o p t}$ (see Appendix A). It can also be shown that for the circular normal distipt bution RMS $=\sqrt{2} \sigma_{\text {opt }}$ (see Appendix A). Since we are considering both specularity effects and surface slope errors in our analysis of individual heliostat module performance, opt must incorporate both these effects, as follows:

$$
\sigma_{o p t}=\sqrt{\sigma_{\Psi}^{2}+\left(2 \sigma_{d}\right)^{2}}
$$

where $\sigma_{\Psi}$ is the standard deviation of the beam width described by the half cone angle (see Figure 2-1) corresponding to specularity effects measured in a single dimension, and $\sigma_{d}$ is the standard deviation of the surface normal error caused by surface deformation measured in a single dimension.** The factor of 2 multiplying of results from the fact that a surface normal error is measured from the actual surface normal to the ideal surface normal, and thus produces twice as large an error in the reflected ray. As will be shown in sections 3 and 4 , this factor of 2 produces greater sensitivity of the delivered energy to surface normal errors than to specularity errors.

In this report $\Psi$ is the half cone angle as measured from the nominal reflected beam (see Figure 2-1). The precise definition of the angle $\Psi$ is important. Because different conventions are sometimes used in analysis and experimental work, the definition of $\Psi$ can lead to confusion. Data from experimental measurements are typically given for the full cone angle a $(=2 \%)$. This difference in conventions has evolved since analytical models normally employ probability distributions in which the half cone angle is the random variable, whereas many two-dimensional experiments measure the energy within the full cone angle. (See Appendix $E$ for more information on specularity and cone size relations.)

*We have defined oot in this way since the parameters in Eq. 2-3 are the optical beam spreading effects, which we vary in this study. Other errors such as tower sway and tracking errors are convolved in an analogous manner, and normal values suggested in DELSOL2 were assumed and used throughout. Further sun size effects and collector size effects that also give rise to beam broadening are handled separately in DELSOL2.

**DELSOL2 requires that surface normal slope errors and specularity errors be combined and expressed in terms of the standard deviations of the surface normal error in the two principal heliostat surface dimensions, $\sigma_{x}^{\prime}$ and $\sigma_{y}^{\prime}$. Thus for a circular normal distribution $\sigma_{o p t} / 2=\sigma_{x}^{\prime}=\sigma_{y}^{\prime}$. 


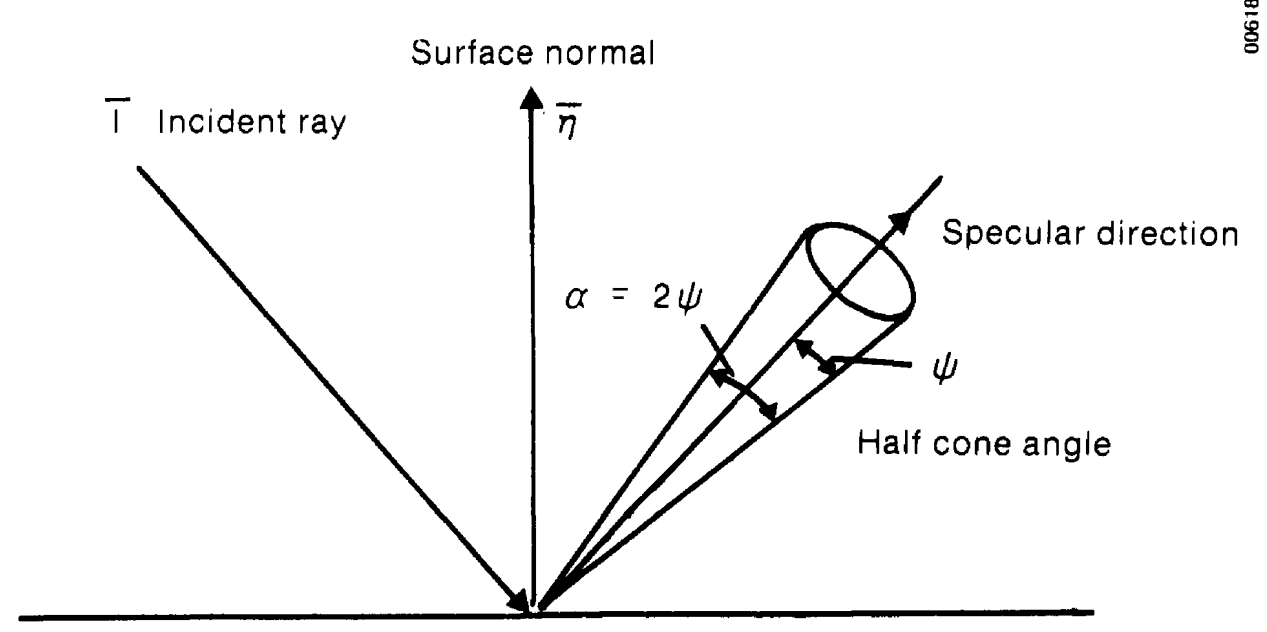

Figure 2-1. Definition of the Specularity Cone in Terms of the Angle about the Nominal Reflected Ray

The formulation of Eq. 2-3 leads fairly naturally to the concept of an "error budget." There are an infinite number of combinations of $\sigma_{d}$ and $\sigma_{\psi}$ that will produce any given value of $\sigma_{o p t}$. This is illustrated in Figure 2-2, where $\sigma_{\psi}$ is plotted against $\sigma_{d}$ for various values of $\sigma_{\text {opt }}$. The importance of this idea is that the parameter that was manipulated in the analysis was $\sigma_{0 p t}$. Thus, when applying these results to a particular heliostat, it will be possible to trade off specularity losses for surface normal (e.8., manufacturing) errors, without impacting performance, as long as $\sigma_{o p t}$ is held constant. Likewise, in our analysis of the heliostats in this report, we can investigate the impact of both types of errors by varying only $\sigma_{\text {opt }} \cdot$

For later discussions one other definition will be helpful. We further partition the surface normal errors into components corresponding to wind and weight induced errors, and to manufacturing induced errors. Again assuming independent normal distributions,

$$
\sigma_{d}^{2}=\sigma_{d 1}^{2}+\sigma_{d 2}^{2},
$$

where $\sigma_{\mathrm{d} 1}$ and $\sigma_{\mathrm{d} 2}$ correspond to the standard deviation of the surface normal errors caused by wind and weight $\left(\sigma_{d}\right)$ and by manufacturing errors $\left(\sigma_{d 2}\right)$, each as measured in a single dimension. Then Eq. 2-3 can be written as

$$
\sigma_{\text {opt }}=\sqrt{\left(2 \sigma_{d 1}\right)^{2}+\left(2 \sigma_{d 2}\right)^{2}+\sigma_{\Psi}{ }^{2}} \text {. }
$$




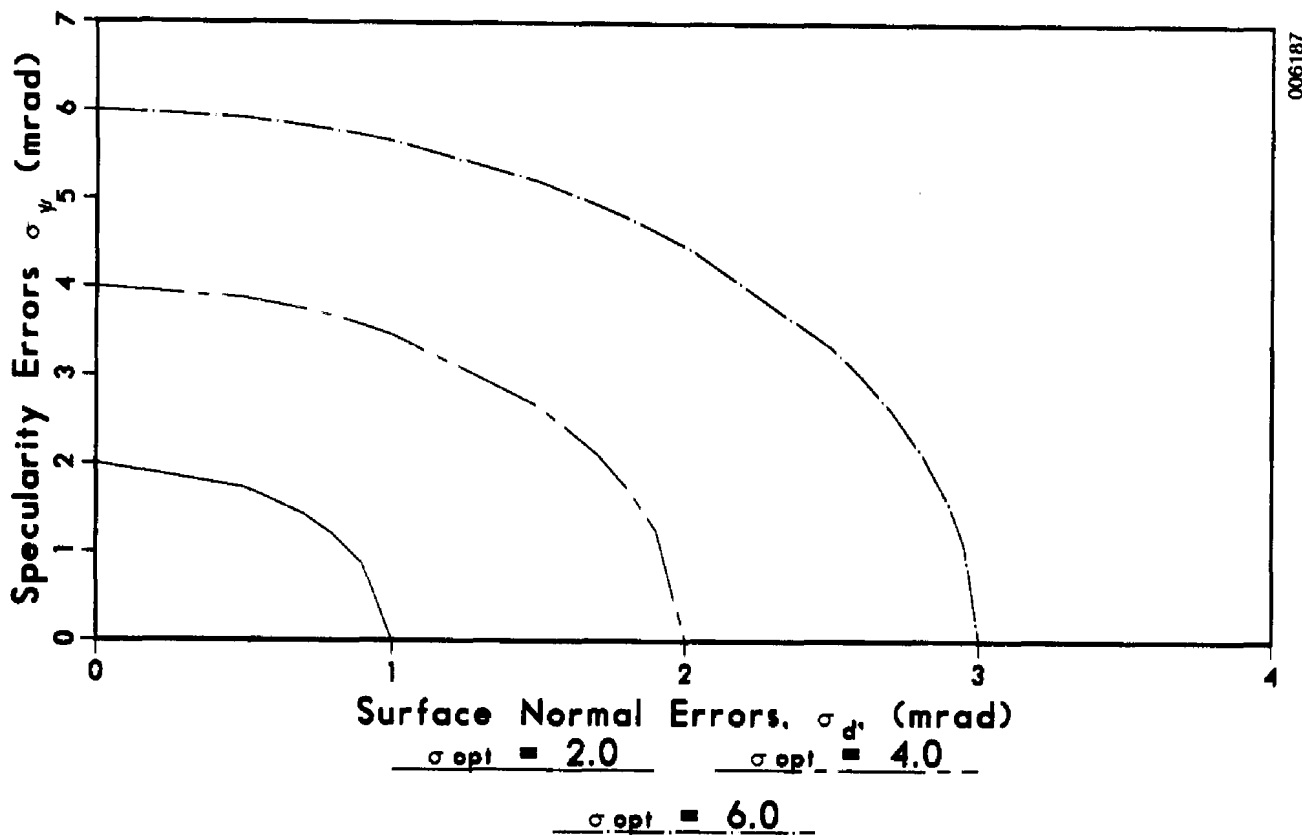

Figure 2-2. Specularity Errors ve. Surface Normal Errors for a Given Level of Total Surface Brror Showing the Tradeoff between Specularity and Surface Brrors

\subsection{ASSUYPTIONS FOR IMDIVIDUAL MODULES}

The basic configurations and characteristics of the individual heliostat modules considered in the system studies are described below, and the corresponding optical performance parameters used in the systems studies are summarized in Table 2-1.

The glass/metal heliostat used as the baseline standard of comparison is a $100-\mathrm{m}^{2}$ rectangular design with twelve facets which are assumed to be focused and canted at the slant range. In previous systems studies [11] that considered this heliostat, the standard deviation corresponding to the combined effects of wind, weight, specularity lost, and manufacturing error is assumed to be given by $\sigma_{o p t}=2.0 \mathrm{mrad}$. If we use a baseline assumption of $\sigma_{\Psi}=0.5 \mathrm{mrad}$ per Pettit et al. [7], Eq. 2-3 implies $\sigma_{d}=0.968 \mathrm{mrad}$. This value then accounts for the combined effects of wind, weight, and manufacturing errors.

The optical performance parameters for the stretched membrane modules are based on the analyses of "typical" (adequate, but not optimal) designs with the structural response characteristics defined by Murphy et al. $[4,6]$. An analysis of the optical performance using these structural response characteristics is presented in Appendix B. The results show that the response to the expected pressure and weight loading drives the design, and also the anticipated optical performance. Appendix $B$ also shows that this response is different for the focused and unfocused modules. The total module weight is shown to be the major design consideration for the asymmetric deformation 
Table 2-1. Baseline Surface Quality Assumptions

\begin{tabular}{lccc}
\hline Error Source & $\begin{array}{c}100-\mathrm{m}^{2} \\
\text { Glass/Metal }\end{array}$ & $\begin{array}{c}100-\mathrm{m}^{2} \text { Focused } \\
\text { Stretched Membrane }\end{array}$ & $\begin{array}{c}100-\mathrm{m}^{2} \text { Unfocused } \\
\text { (Ideally Flat) } \\
\text { Stretched Membrane }\end{array}$ \\
\hline $\begin{array}{c}\text { م, Hemispherical } \\
\text { reflectivity }\end{array}$ & 0.89 & 0.89 & 0.89 \\
$\begin{array}{c}\text { opt Wind \& Weight } \\
\text { manufacturing/assembly } \\
\text { specularity (mrad) }\end{array}$ & 2.0 & 2.0 & $3.46^{\mathrm{b}}$ \\
\hline
\end{tabular}

${ }^{a}$ Assumes a 0.05 allowance for average dirt accumulation. The reflectance for a clean mirror is assumed to be 0.94 .

${ }^{b}$ Assumed relative to the perfectly flat state.

induced by noncontinuous supports. However, for the axisymmetric membrane deformation of the unfocused module, the wind effect is the major design driver. This axisymmetric deformation, however, can be eliminated through active control in focused modules, which is an important assumption used for the focused modules.

For the focused module we found the RMS value of the slope corresponding to wind- and weight-induced errors to be about $0.50 \mathrm{mrad}$ (see Appendix $B$ ). This results in a corresponding standard deviation of $\sigma_{\mathrm{d} 1}=0.50 / \sqrt{2}$. This would imply by Eq. $2-5$, assuming a value of $\sigma_{0 p t}=2.0 \mathrm{mrad}$ and $\sigma_{\psi}=0.5 \mathrm{mrad}$, that the standard deviation of manufacturing errors is $\sigma_{d 2}=0.927 \mathrm{mrad}$. This appears quite reasonable relative to the 8 lass metal heliostats where the composite $\sigma_{d}=0.968 \mathrm{mrad}$, since the bulk of the surface errors can be allocated to the manufacturing error.

For the unfocused modules, which are assumed to be flat in their perfect condition, we found (see Appendix B) the upper bound of the RMS value of the slope error corresponding to wind and weight induced errors to be about 2.06 mrad.* This results in a corresponding $\sigma_{\mathrm{dl}}=2.06 / \sqrt{2}=1.46 \mathrm{mrad}$. If we then assume $\sigma_{\Psi}=0.5 \mathrm{mrad}$, and the same manufacturing error as that corresponding to the focused modules, $\left(\sigma_{\mathrm{d} 2}=0.927 \mathrm{mrad}\right)$, then $\sigma_{\rho p t}=3.46 \mathrm{mrad}$. This was used as the baseline value for the unfocused designs (gee Table 2-1).

Consistent with the analyses provided in Murphy [5] and Murphy et al. [6] and in Appendix B, the following assumptions are made:

- The stretched membrane concepts are assumed to be of the double membrane design, to have a circular shape, and to have three evenly spaced supports around the circumference whether the module is focused or not. The double membrane selection for the unfocused design is consistent with the structural efficiency arguments in Murphy [5] and Murphy et al. [6].

$* \mathrm{RMS}^{2}=\mathrm{RMS}_{\mathrm{AX}}^{2}+\mathrm{RMS}_{\mathrm{AS}}^{2}=2^{2}+(0.5)^{2}$, where the subscripts AX and AS correspond to the axisymmetric and asymmetric contributions, respectively. 
- Various module sizes are considered for the stretched membrane concept but the baseline assumed is $100 \mathrm{~m}^{2}$ (11.28-m diameter). It should be noted that the error approximations established in Appendix $B$ and noted in Table 2-1 are based on extensive analyses of a $78-\mathrm{m}^{2}$ (10.0-m diameter) design. It is not anticipated that these approximations should change significantly with the $11.28-\mathrm{m}$ design. However, for sizes significantly smaller the current error estimates will be conservative. This is especially true for the axisymmetric deformations considered with the nonfocused designs.

- Designs using steel for both the frame and membranes were assumed since it is the most common construction material. However, the lighter materials appear to offer some advantage, and the error approximations given here may be somewhat high for these lighter designs.

- For the unfocused design the error caused by the axisymmetric deformation of the reflector membrane must be considered, and is added in quadrature to the asymmetric error caused by the out-of-plane frame distortion. A reasonable upper bound estimate for this axisymmetric error is assumed to be RMS $_{A X}=2$ mrad per the analysis presented in Appendix $B$. The error is assumed to be measured relative to a perfectly flat condition, and thus no credit for the weight induced focusing of the reflector membrane is considered. Since a number of other conservative assumptions are also made in Appendix B, the performance appears to be bounded by considering RMS $\mathrm{AX}$ to be between zero and $2 \mathrm{mrad}$ for the axisymmetric displacements.

- For the focused stretched membrane heliostat, the control scheme proposed by SNLL is assumed to be operative, and is further assumed to be effective in controlling the axisymmetric membrane deformations to negligible levels. In the SNLL approach, an actively controlled pressure/ deformation mechanism controls the pressure level within the plenum chamber separating the two parallel membranes, such that the axisymmetric deformation in the reflector membrane caused by wind induced pressure is exactly balanced by the internal pressure within the chamber. Thus in this case, only the asymmetric deformation caused by the frame deformation between the supports is considered (i.e., $\mathrm{RMS}_{\mathrm{AX}}=0$ and the error corresponding to wind and weight loading is assumed to be RMS $=$ RMS $_{A S}=0.5 \mathrm{mrad}$ ). 
SECTION 3.0

\section{SYSTEMS STUDIES ON THE FOCUSED STRETCHED MEMBRANE MODULE}

In this section we describe the systems tradeoffs for the focused stretched membrane heliostats relative to the $100-\mathrm{m}^{2}$, second-generation glass/metal heliostats. The performance issues we investigate include the sensitivity of system cost and performance to (1) stretched membrane module size, (2) surface normal errors on the stretched membrane reflective surface, (3) loss of specularity on the stretched membrane reflective film, (4) loss of reflectivity for the stretched membrane module, and (5) cost of the stretched membrane module. The tradeoffs between focused and unfocused modules are discussed in Section 4.0.

\subsection{ASSUMPTIONS AND GROUND RULES}

In many of the comparisons we normalize the energy delivered by the stretched membrane heliostat with the energy delivered by the corresponding baseline $100-\mathrm{m}^{2} \mathrm{glass} / \mathrm{metal}$ heliostat. The energy delivered is always described on an annual basis and given per square meter of the heliostat being considered. Only IPH systems are considered, and system temperature refers to the temperature of the energy delivered at the base of the tower, which is assumed to be the same temperature as that at the outlet of the receiver. In addition the following assumptions are made.

- The heliostat optical performance characteristics are as described in Section 2.0 .

- We consider system sizes of 75,225 , and $450 \mathrm{MW}_{\text {th }}$ with the corresponding approximate field sizes of $100,000,300,000$, and $700,000 \mathrm{~m}^{2}$, respectively.

- Average absorber temperatures of $300^{\circ}, 600^{\circ}$, and $900^{\circ} \mathrm{C}$ were used in the analysis. Fur the purpose of comparison, these are listed as delivery temperatures of $450^{\circ}, 750^{\circ}$, and $1050^{\circ} \mathrm{C}$, respectively. These delivery temperatures assume a nominal temperature rise of $300^{\circ} \mathrm{C}$ across the absorber.

- Conventional single cavity, open aperture receivers coupled to a north field are assumed, with receiver loss mechanisms similar to those considered in the earlier study, The Performance of High Temperature Central Receiver Systems [11].

- Although the general characteristics of the plant were the same from system to system, the details were optimized individually for each combination of plant size, temperature, and heliostat type (glass/metal, focused stretched membrane, and unfocused stretched membrane). The parameters that were optimized included the tower height, the field size and layout, the receiver height and depth, and the aperture dimensions.

- The optimizations were based only on performance. The optimization procedure is described in Appendix $C$, and involves the iterative use of three computer codes (DELSOL2 [10], RADSOLVER [12], and SHAPEFACTOR [13]) for each temperature and plant size considered. A comparison of our 
optimization procedure with a combined cost/performance optimization is presented in Appendix D.

- The field performance parameters required by DELSOL2, but not described with respect to the optical performance characteristics above, are assumed to be the DELSOL2 default values. These parameters include error estimates for tower sway tracking errors, and sun angle effects.

- For the system cost tradeoffs a levelized cost methodology [14] is employed, using the parameters given in Table 3-1. These parameters are consistent with the current draft Five-Year Plan for the Solar Thermal Program [14].

- The component cost assumptions are the same as those in the MYPP [14] except where noted. Specifically, sfretched membrane heliostats are assumed to cost either $\$ 50 / \mathrm{m}^{2}$ or $\$ 100 / \mathrm{m}^{2}$.

Table 3-1. Levelized Energy Cost Assumptions

\begin{tabular}{lc}
\hline Costs (all costs in $\$ / \mathrm{m}^{2}$ of heliostat) \\
Heliostats & $\$ 50$ or $\$ 100$ \\
Receiver & $\$ 45$ \\
Transport & $\$ 25$ \\
Balance of Plant & $\$ 50$ \\
Annual O\&M & $\$ 5$ \\
Indirects and Contingencies* & $20 \%$ \\
Financial Parameters & \\
Discount Rate (real) & 0.10 \\
Fixed Charge Rate & 0.1334 \\
Capital Recovery Factor & 0.1175 \\
Construction Time & \\
Adjustment Factor & 1.1033 \\
\hline
\end{tabular}

*The total capital cost of the system was calculated by summing the costs for the individual components, and increasing that value by $20 \%$ to account for indirect costs and contingency factors [14]. 


\subsection{RESULTS FOR FOCUSED MEMBRANE MODULES}

In this section we present sensitivity results for the numerous heliostat parameters and system costs. For each parameter examined we first address performance issues and then introduce the cost/performance sensitivities.

A sense of the absolute energy delivered by the two types of heliostats is given in Figure 3-1, which shows the annual energy per unit area of heliostat as a function of delivery temperature for the glass/metal heliostats and for the focused stretched membrane heliostats each with similar optical qualities. For comparison purposes the results from DeLaquil and Anderson [11] are also shown, and one point from the IPH study in Eiker et a1. [15] corresponding to an external receiver is also shown. It is seen that the stretched membrane heliostat has a nearly uniform slight advantage over that of the standard glass metal heliostat. This slight advantage is the result of the round shape of the stretched membrane heliostat versus the rectangular shape of the glass/ metal heliostat. Although the field density is higher, there is slightly less shading and blocking with the stretched membrane because of the corners on the rectangular heliostat. Thus it is possible that a single stretched membrane heliostat could have slightly poorer optical quality than the glass/metal concept and still produce equal field performance. We address this point below.

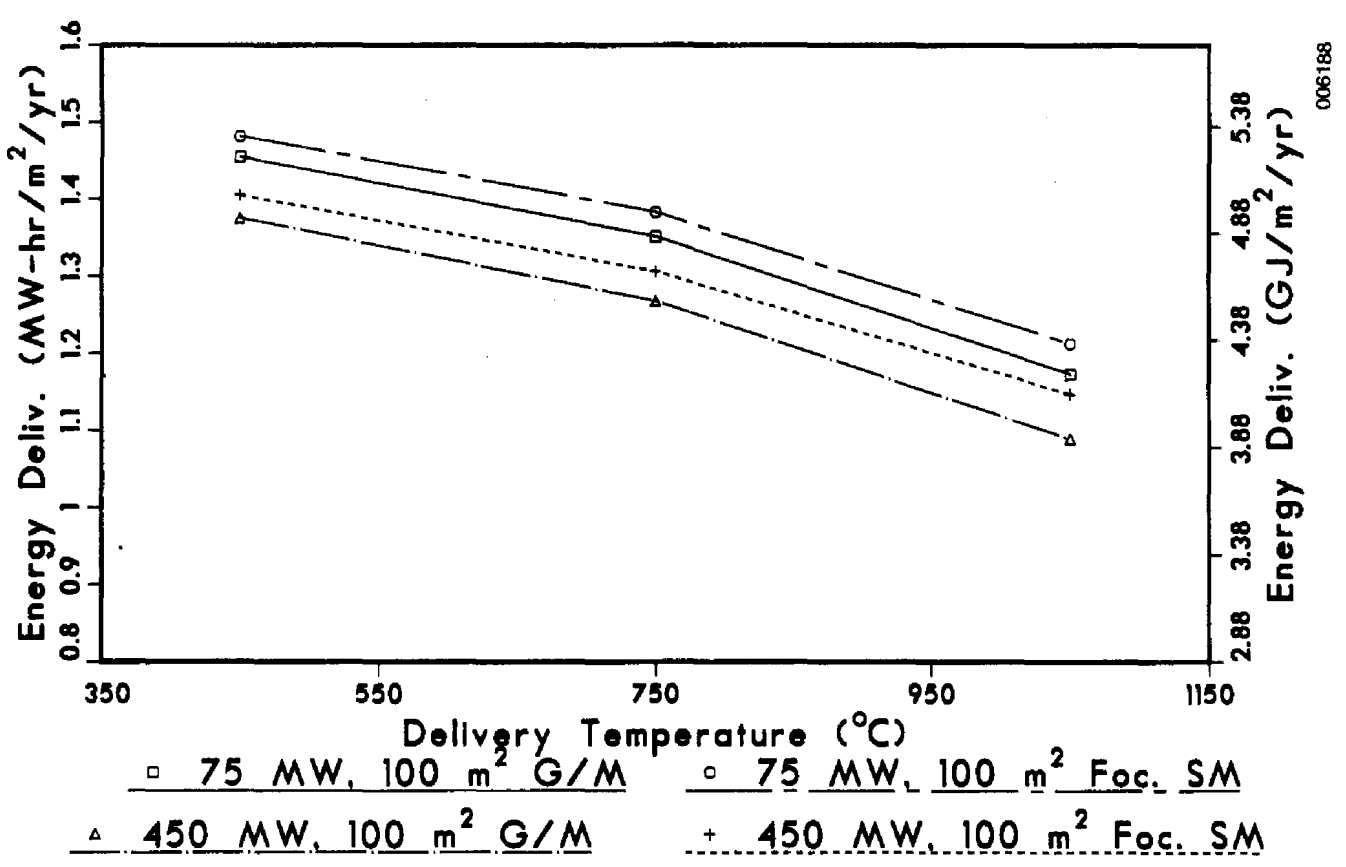

Figure 3-1. Annual Delivered Energy per $\mathrm{m}^{2}$ of Heliostat for Pocused Stretched Membrane Heliostats and Current 100-m² Glass/ Metal Heliostats at Two Plant Sizes as a Function of Temperature 
In Figure 3-2 the annual energy delivery for the focused stretched membrane heliostats is normalized to that for the glass/metal heliostats and plotted as a function of the stretched membrane heliostat area.* These results show that within the limitations of the DELSOL2 [10]*t code and the range of plant sizes studied, there is no significant size effect for the focused stretched membrane heliostat. This finding was used to simplify our subsequent analysis and comparisons in which we consider only $100-\mathrm{m}^{2}$ focused stretched membrane heliostats. (It should be noted that size is an important consideration for the unfocused stretched membrane. This is discussed in Section 4.0.)

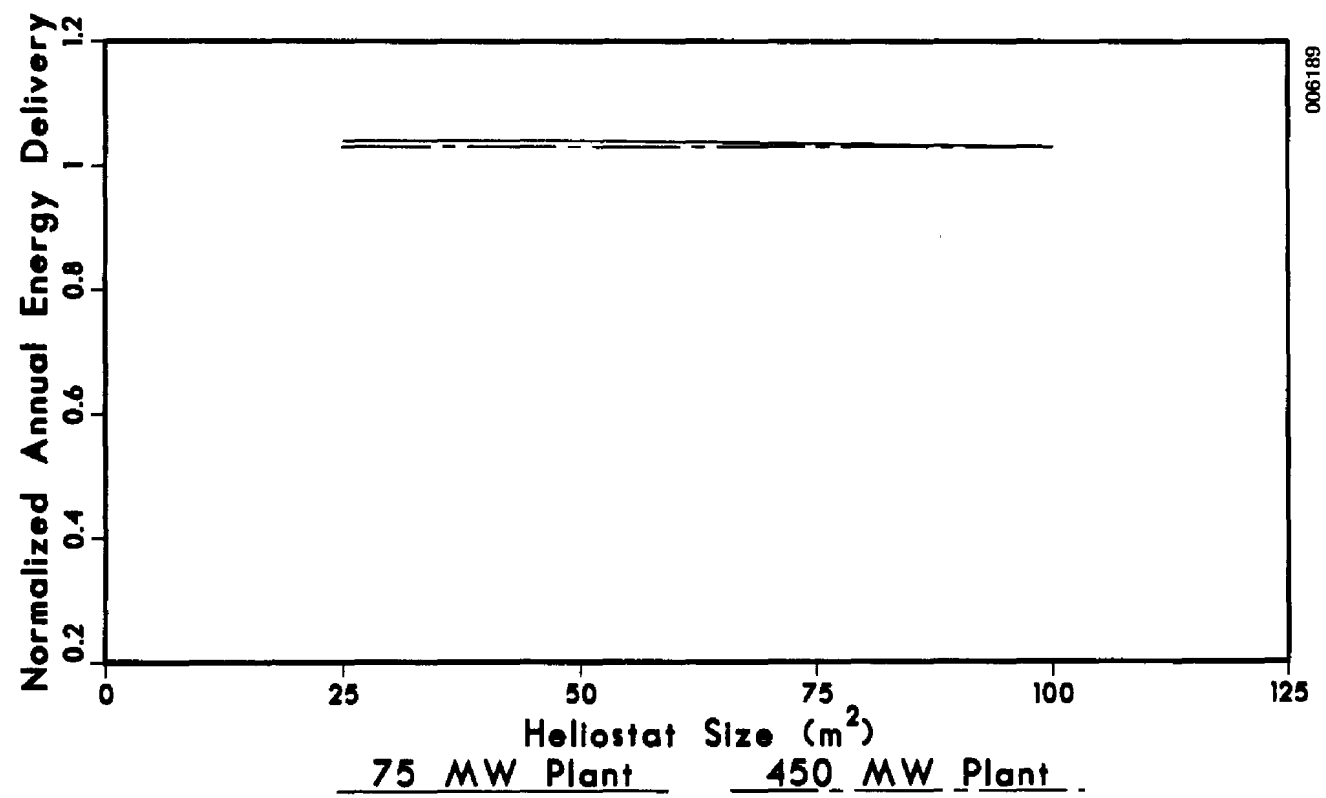

Figure 3-2. Annual Energy Delivered as a Function of Focused Stretched Membrane Heliostat Area. The delivered energy results are normalized with the $100-\mathrm{m}^{2} \mathrm{glass} / \mathrm{metal}$ focused heliostat.

*This is a common form of presentation in this report. The resulting ratio will be referred to as the "Normalized Annual Energy Delivery."

weDELSOL2 [10] does not handle the astigmatic effect correctly. However, when the focused stretched membrane is of the same order of size as the $100-\mathrm{m}^{2}$ glass/metal concepts, the relative effects should be similar in magnitude for the two concepts. For smaller sizes of stretched membranes there may be another slight advantage for the stretched membrances when compared to the glass/metal concepts.

There is evidence in an earlier report [11] that for very small plant sizes ( $5 \mathrm{MW}$ ) the size of focused heliostats does have a somewhat greater effect on the performance. 
Figures 3-3 and 3-4 show the performance sensitivity of the stretched membrane system as the surface error is increased beyond the base line levels. For the $100-\mathrm{m}^{2}$ focused stretched membrane heliostats and $100-\mathrm{m}^{2}$ glass/metal modules, Figures $3-3$ and $3-4$ show the normalized annual energy as a function of the stretched membrane heliostat surface error for several values of the delivery temperature. Figure $3-3$ is for a $75-\mathrm{MW}_{\mathrm{th}}$ plant, and Figure $3-4$ for a $450-\mathrm{MW}_{\text {th }}$ plant. Both plant sizes show a significant performance drop-off as the total surface error is increased from the nominal value of $\sigma_{0 p t}=2.0$ to $\sigma_{\text {opt }}=$ 4.80. Note that although there is much less effect with temperature for a given value of $\sigma_{o p t}$, the high temperature systems are more sensitive to a decrease in the optical quality than the lower temperature systems. This is because of the tighter focusing requirements caused by the smaller apertures required at higher temperatures.

The values of $\sigma$ in Figures $3-3$ and $3-4$ correspond to the RMS error levels shown in Table $3 \underline{\underline{p}}$. Table $3-2$ also shows the corresponding value of $\sigma_{d}$, the standard deviation due to surface deformation (computed from Eq. 2-2, assuming the standard deviation due to specularity, $\sigma_{\psi}$, is $0.5 \mathrm{mrad}$ ). A comparison of the first two cases ( $\sigma_{o p t}=2.0 \mathrm{mrad}$, the base case, and $\sigma_{o p t}=3.46 \mathrm{mrad}$ ) indicates that a $77 \%$ increase $(1.712 / 0.968)$ in the standard deviation of the surface slope error, $\sigma_{d}$, yields only a small decrease $(<5 \%)$ in the annual energy delivered.

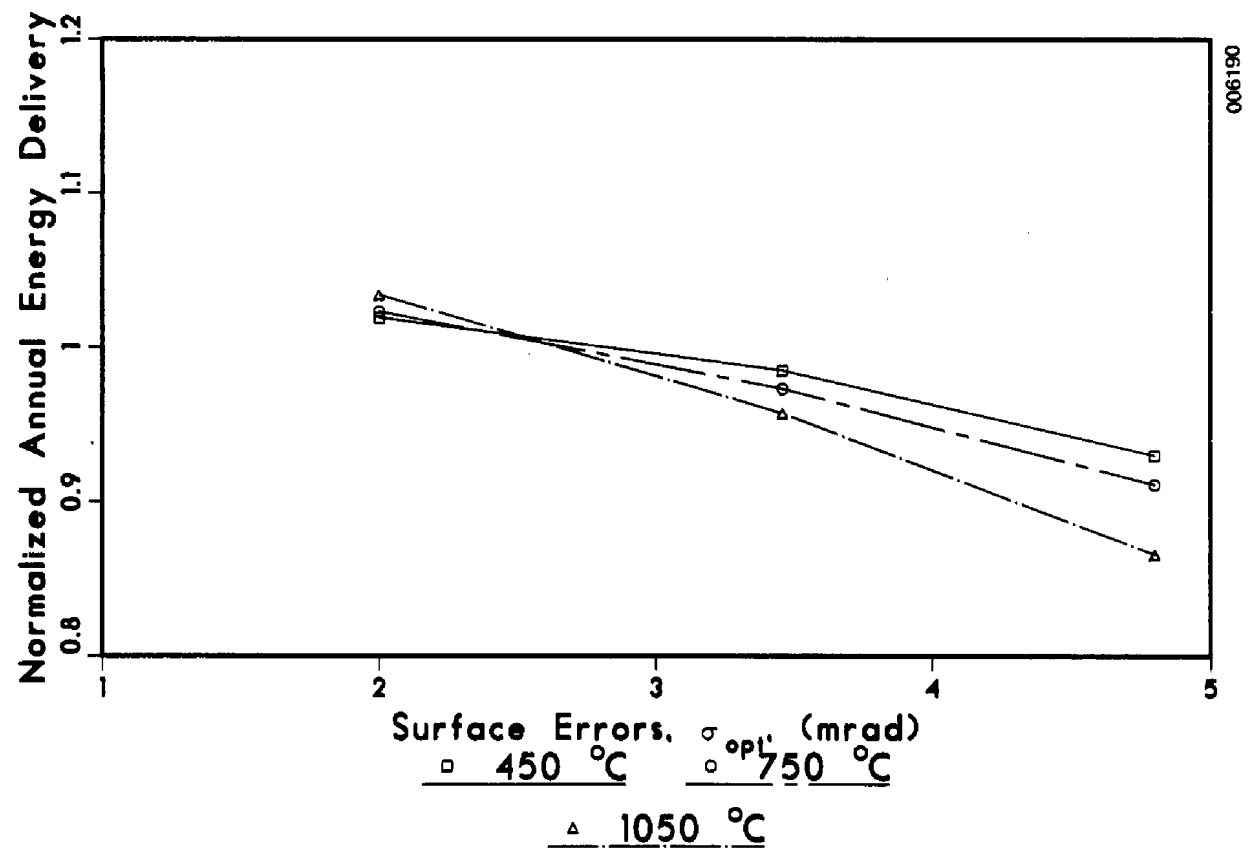

Figure 3-3. Annual Energy Delivered by Stretched Membrane Heliostat Systems with Various Levels of Surface Error, Normalized by the Annual Energy Delivered by State-of-the-Art Glass/Metal Heliostat System for a 75-MW plant. Both heliostat types and had reflective areas of $10 \mathrm{~cm}^{2}$, opt for the glass/metal heliostat was assumed to be $2.0 \mathrm{mrad}$. The surface errors are assumed to include both surface waviness and specularity effects. 


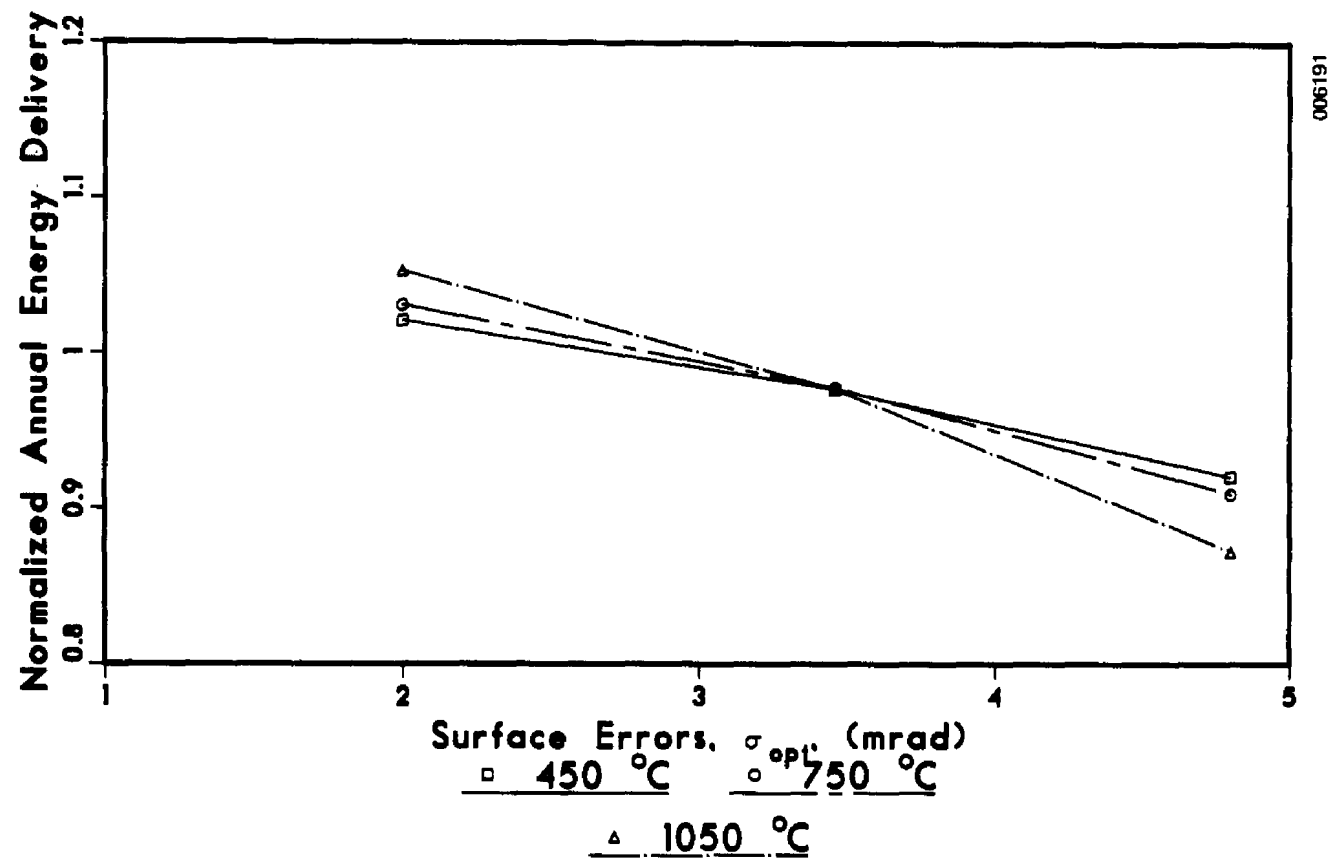

Figure 3-4. Annual Energy Delivered by Stretched Membrane Heliostat Systems with Various Levels of Surface Brror Normalized by the Annual Energy Delivered by State-of-the-Art Glass/Metal Heliostat System for a 450-MW Plant. Both heliostat types had reflective areas of $100 \mathrm{~m}^{2}$, and $\sigma_{0 p t}$ for the glass/metal heliostat was assumed to be $2.0 \mathrm{mrad}$. The surface errors are assumed to include both surface waviness and specularity effects.

Table 3-2. Brror Parameters ${ }^{a}$ of Eigures 3-3 and 3-4

\begin{tabular}{cccc}
\hline$\sigma_{\text {opt }}$ & RMS & $\sigma_{d}{ }^{b}$ & $\sigma_{\Psi}{ }^{c}$ \\
\hline 2.00 & 2.83 & 0.968 & 0.50 \\
3.46 & 4.89 & 1.7121 & 2.87 \\
4.80 & 6.79 & 2.387 & 4.39 \\
\hline
\end{tabular}

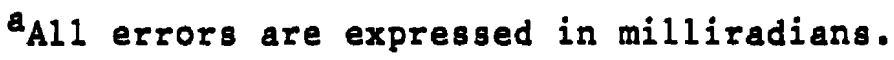

bAssumes $\sigma_{\Psi}=0.5 \mathrm{mrad}$.

$c_{\text {Assumes }} \sigma_{d}=0.968 \mathrm{mrad}$.

In Figure 3-5 the levelized energy cost (LEC) is shown as a function of $\sigma_{0 p t}$ for a $75-\mathrm{MW}_{\text {h }}$ plant at $750^{\circ} \mathrm{C}$ and $1050^{\circ} \mathrm{C}$. Similar information is shown ${ }^{\circ}$ Figure 3-6 for a 45 $\mathrm{M}-\mathrm{MW}_{\text {th }}$ plant. Here two values of stretched membrane heliostat cost $\left(\$ 50 / \mathrm{m}^{2}\right.$ and $\left.\$ 100 / \mathrm{m}^{2}\right)$ are used, and for comparison the LEC cost assuming the baseline glass/metal heliostats (at $\$ 100 / \mathrm{m}^{2}$ and $750^{\circ} \mathrm{C}$ ) is shown. It is seen that the surface error (as measured by $\sigma_{o p t}$ ) can be 
increased nearly $73 \%$ relative to the glass/metal value, and stretched membrane heliostats costing $\$ 100 / \mathrm{m}^{2}$ would still be cost effective relatiye to the glass/metal units. Further, for stretched membranes costing $\$ 50 / \mathrm{m}^{2}$, surface error increases of more than $240 \%$ relative to the glass/metal values would still result in cost effectiveness for the stretched membrane concept.

In Figure 3-7 the normalized annual energy (relative to the glass/metal system) from a $75-\mathrm{MW}_{t h}$ plant is shown as a function of the standard deviation of the specularity errors, $\sigma_{\psi}$, of the stretched membrane heliostats. In Figure 3-8 the levelized cost of energy is shown as a function of $\sigma_{\Psi}$ for two stretched membrane costs $\left(\$ 100 / \mathrm{m}^{2}\right.$ and $\left.\$ 50 / \mathrm{m}^{2}\right)$ and the same plant considered in Figure 3-7. In both of these plots the baseline surface normal errors of $\sigma_{d}=0.968 \mathrm{mrad}$ are assumed to be constant. It is seen that the system is much more tolerant of specularity error than of surface deformation as expressed by $\sigma_{d}$, which follows from Eq. 2-3. For instance at $600^{\circ} \mathrm{C}$ increasing the specularity error standard deviation from $\sigma_{\psi}=0.5 \mathrm{mrad}$ to $4.00 \mathrm{mrad}$ while holding $\sigma_{d}=0.968 \mathrm{mrad}$ has the same effect as changing $\sigma_{d}=0.968 \mathrm{mrad}$ to $\sigma_{d}=2.21 \mathrm{mrad}$ while holding $\sigma_{\psi}$ constant at $0.5 \mathrm{mrad}$.

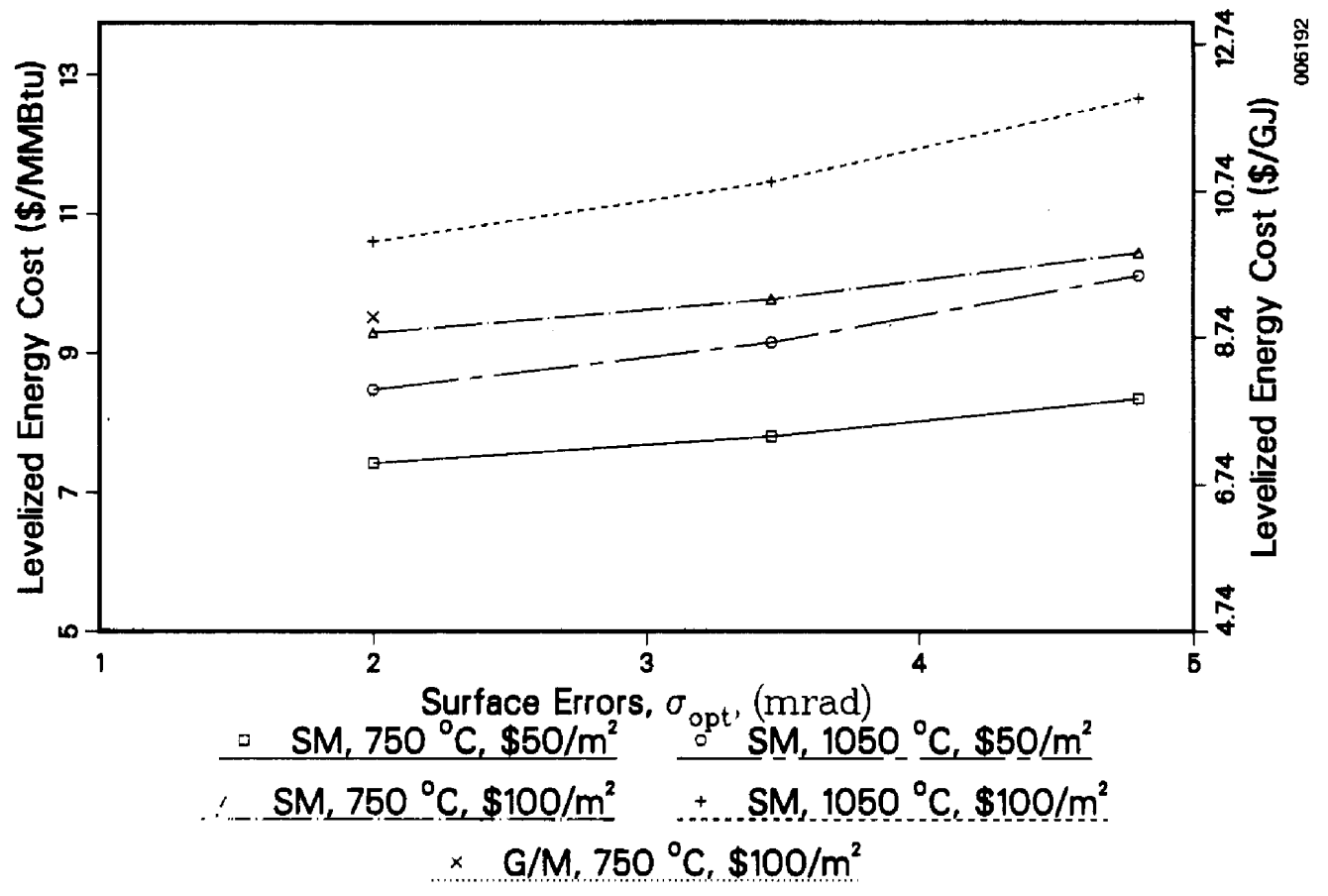

Figure 3-5. Levelized Annual Energy Cost as a Function of Surface Error for the 75-MW Plant (as in Figure 3-3) at Two Delivery Temperatures and Two Heliostat Costs. The LEC for the baseline glass/metal heliostat system (at $750^{\circ} \mathrm{C}$ and $\$ 100 / \mathrm{m}^{2}$ ) is shown for comparison. 


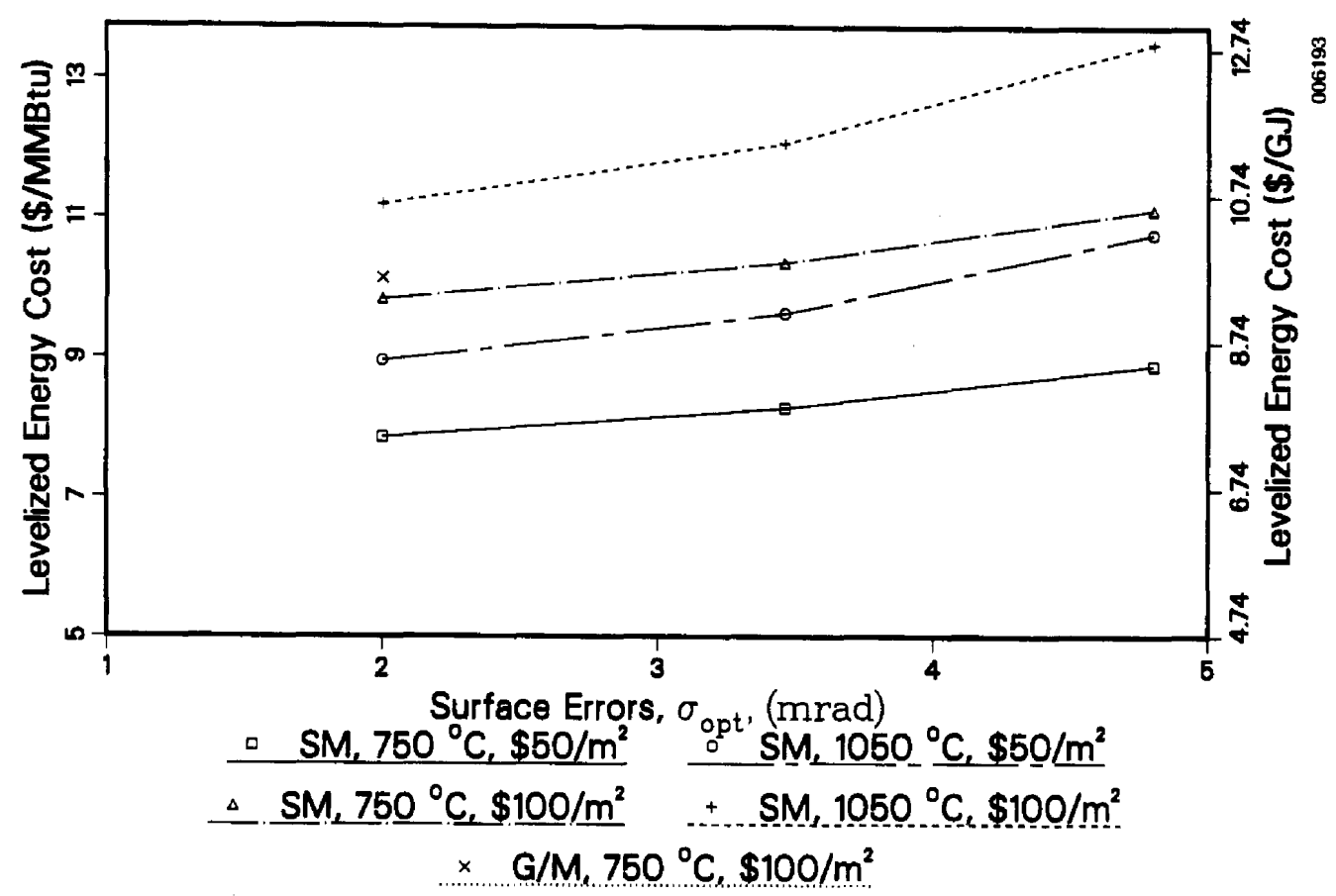

Figure 3-6. Levelized Annual Energy Cost as a Function of Surface Error for the 450-MU plant (as in Figure 3-4) at Two Delivery Temperatures and Two Heliostat Costs $\left(\$ 50 / \mathrm{m}^{2}\right.$ and $\left.\$ 100 / \mathrm{m}^{2}\right)$. The LEC for the baseline glass/metal heliostat system (at $750^{\circ} \mathrm{C}$ and $\$ 100 / \mathrm{m}^{2}$ ) is shown for comparison.

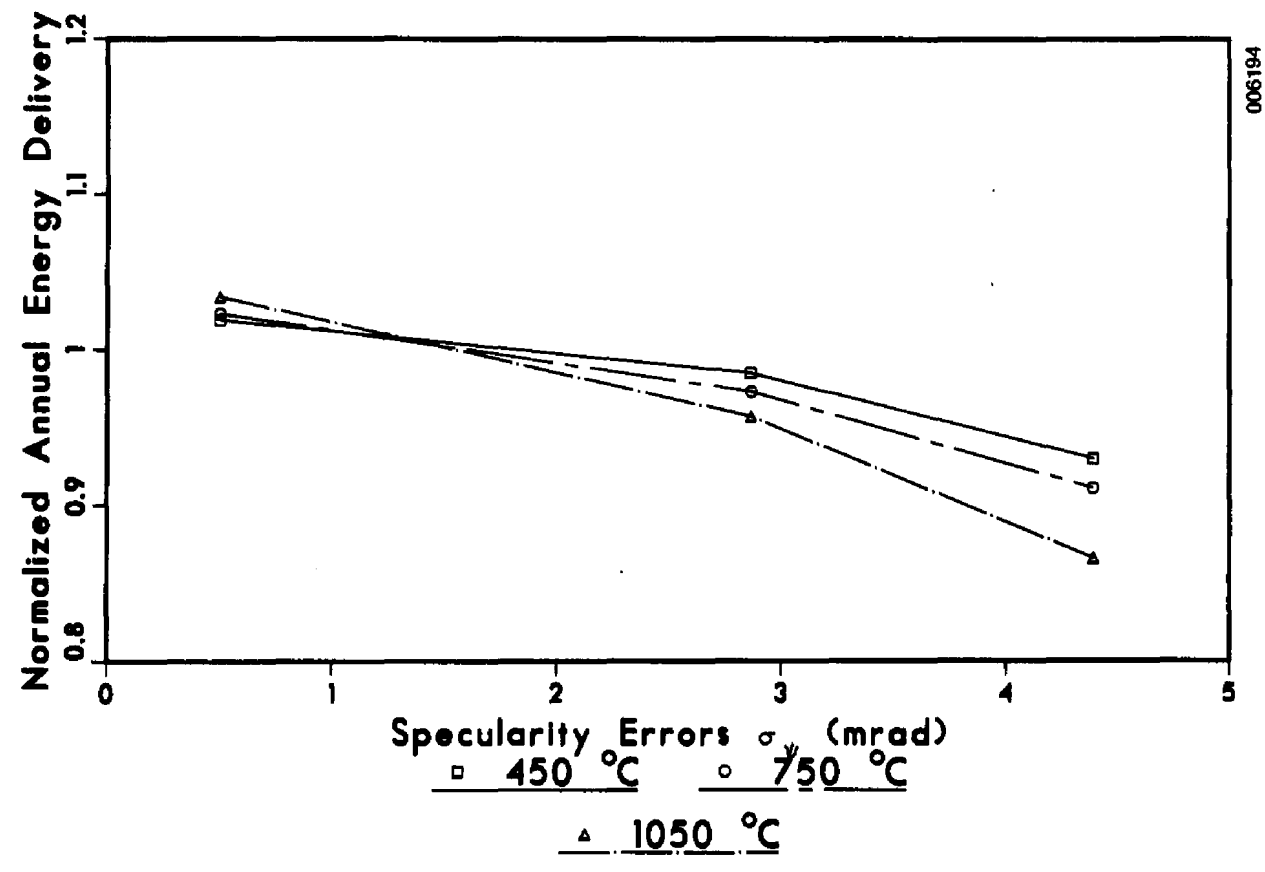

Figure 3-7. Normalized Annual Energy Delivery from Focused Stretched Membrane Heliostats as a Function of the Standard Deviation of the Specularity Errors for a 75-MW Plant at Three Delivery Temperatures 


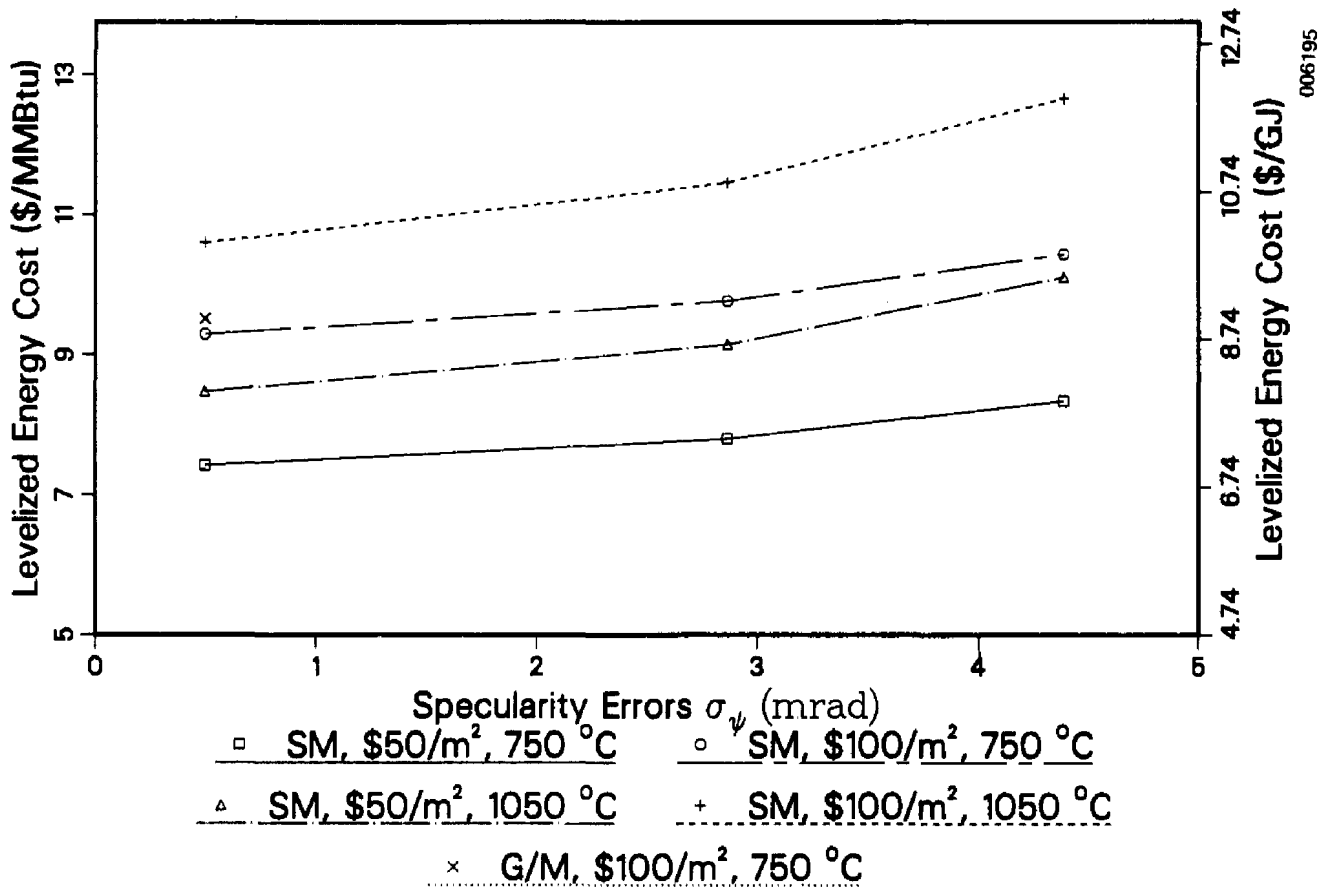

Figure 3-8. Levelized Annual Energy Cost as a Function of the Standard Deviation of the Specularity Errors for the 75-MW Plant at Two Delivery Temperatures and Two Heliostat Costs Assuming $\sigma_{\psi}=0.968 \mathrm{mrad}$. The LEC for the baseline glass/metal heliostat system (at $750^{\circ} \mathrm{C}$ and $\$ 100 / \mathrm{m}^{2}$ ) is shown for comparison.

The specularity errors shown in Figures $3-7$ and 3-8 in terms of $\sigma_{\psi}$ can also be expressed in terms of the half cone angle $\Psi$ for which the cone encompasses some fraction $G(\Psi)$ of the total reflected energy. Assuming a circular normal probability distribution for the specularity errors, the value of $\Psi$ can be found from $G(\Psi)$ by the equation (see Appendix E)

$$
\Psi=\sigma_{\Psi} \sqrt{-2 \ln [1-G(\Psi)]} \text {. }
$$

For example, for the base case value of $\sigma_{\psi}=0.5 \mathrm{mrad}$, the half cone angle $\Psi$ that includes $90 \%$ of the reflected energy $[G(\Psi)=0.9$ ] is $1.07 \mathrm{mrad}$ (assuming no surface deformation errors). Figure 3-9 uses this representation of the specularity errors to show the relationship between specularity errors and heliostat costs. The data in Figure 3-9 have been taken from Figure 3-8 for the $75-\mathrm{MW}$ th plant with $750^{\circ} \mathrm{C}$ delivery temperature. Figure 3-9 shows that if for specularity errors the half cone angle necessary to capture $90 \%$ of the reflected energy increases from $1 \mathrm{mrad}$ to almost $10 \mathrm{mrad}$ (or, equivalently, $\sigma_{\psi}$ increases from $0.5 \mathrm{mrad}$ to almost $5 \mathrm{mrad}$ ), the cost of the heliostats must only decrease from $\$ 103 / \mathrm{m}^{2}$ to $\$ 74 / \mathrm{m}^{2}$ to yield a constant LEC of $\$ 8 / 10^{6}$ Btu. Thus, as stated earlier, system costs and performance are not very sensitive to specularity errors.

Hemispherical reflectivity effects are illustrated in Figures 3-10 and 3-11. In Figure 3-10 the normalized annual delivered energy by the focused stretched membrane heliostats in a $75-\mathrm{MW}_{\text {th }}$ plant operating at $600^{\circ} \mathrm{C}$ is shown as a function of reflectivity, and the corresponding levelized energy cost is shown in 


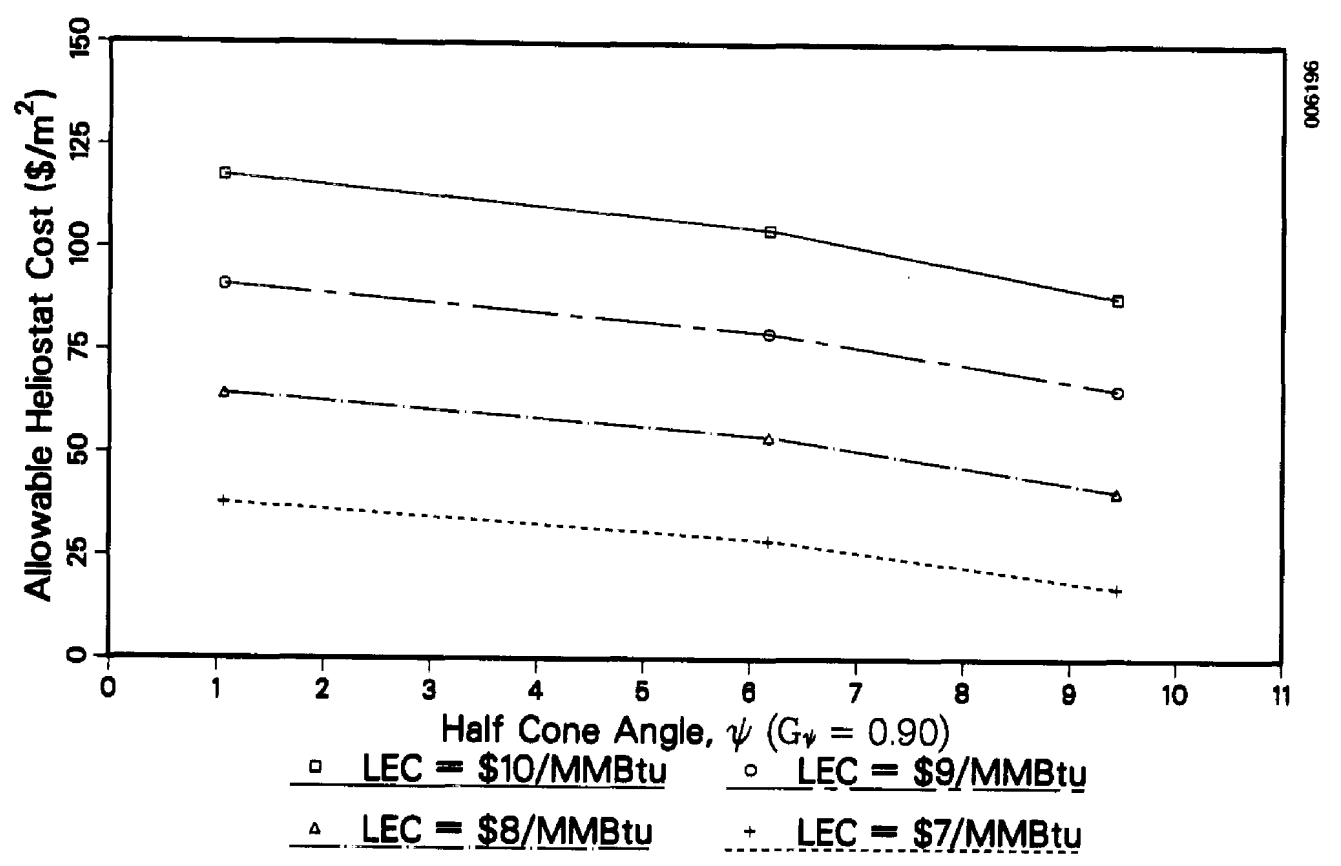

Pigure 3-9. Allowable Heliostat Cost as a Function of the Specularity Half Cone Angle for Several Legels of Levelized Energy Cost for a 75-1W th Plant Using 100- $\mathrm{m}^{2}$ Focused Stretched Membrane Heliostats at a Delivery Temperature of $750^{\circ} \mathrm{C}$. This is the heliostat cost that allows you to maintain the given LEC despite increasing specularity errors.

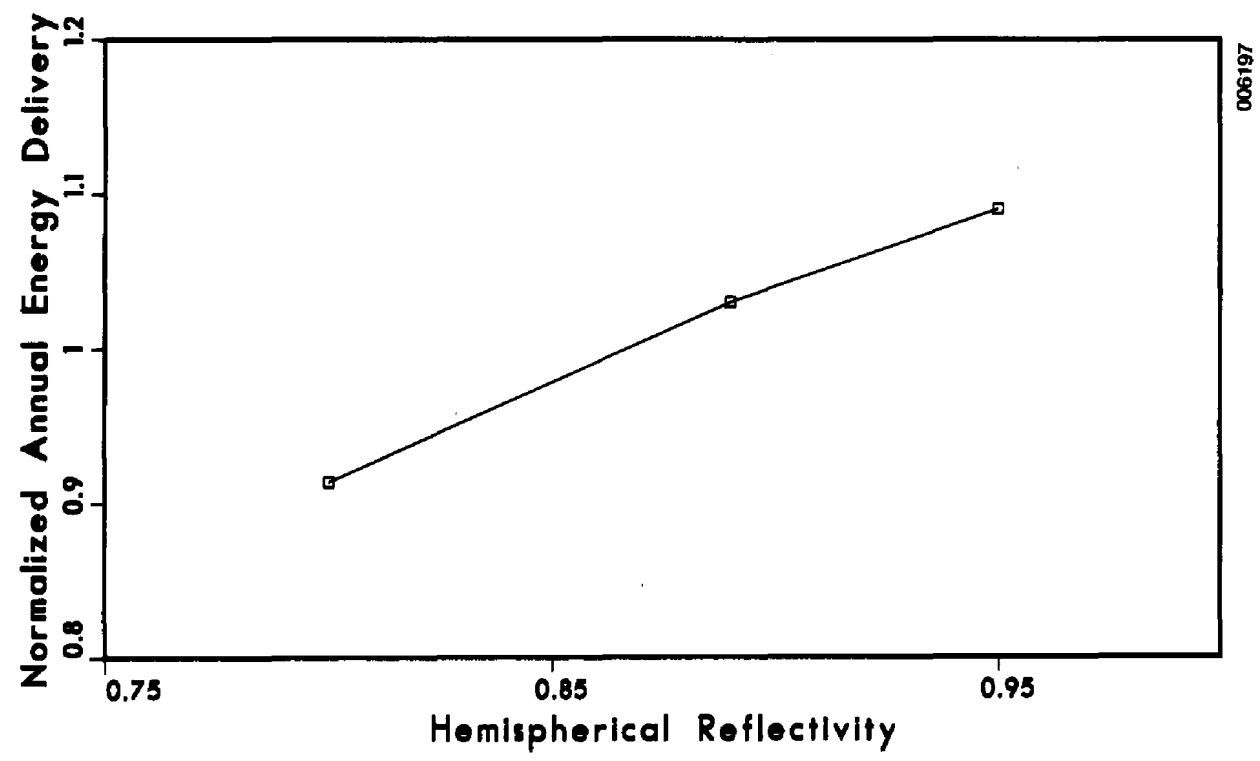

Figure 3-10. Normalized Annual Energy Delivery from Focused Stretched Membrane Heliostats as a Eunction of the Hemispherical Reflectivity for a $75-\mathrm{MW}$ Plant at $750^{\circ} \mathrm{C}$ Delivery Temperature 


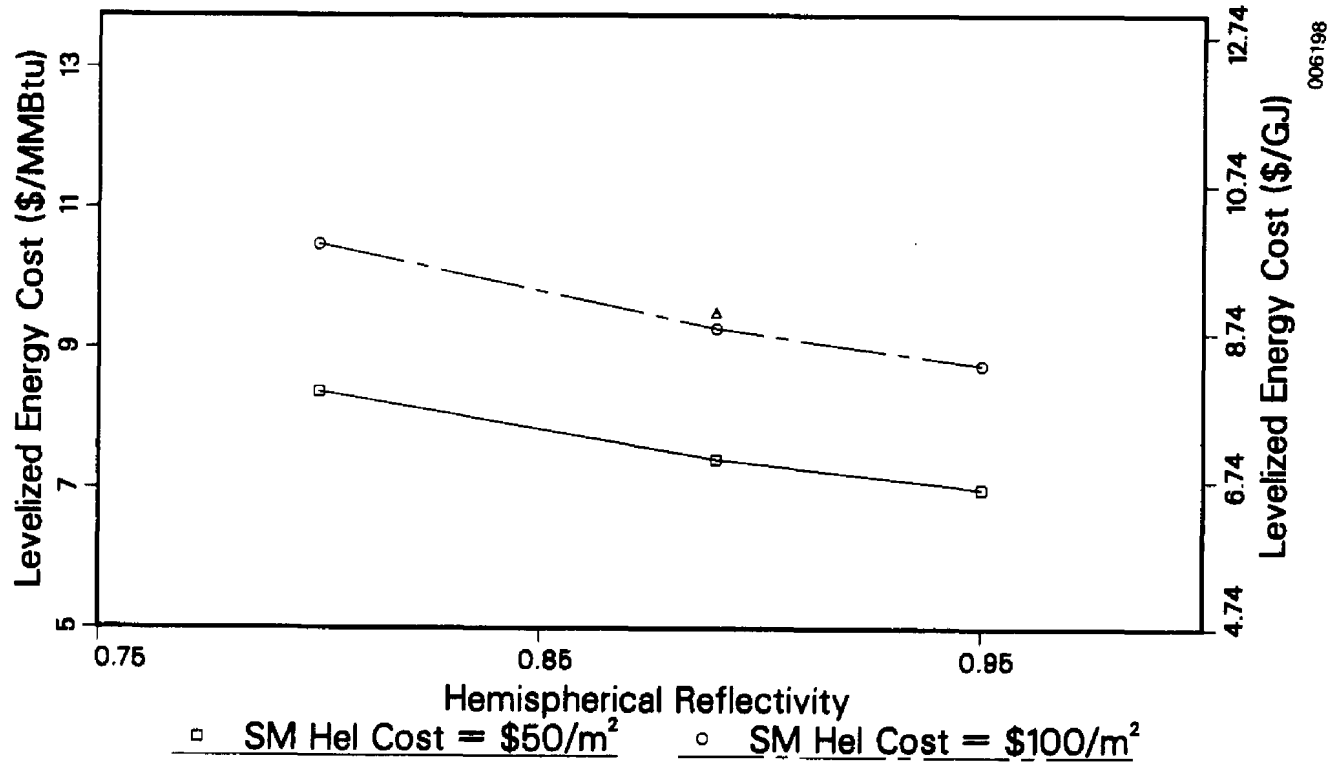

$\triangle$ Baseline $\mathrm{G} / \mathrm{M}$ Hel Cost $=\$ 100 / \mathrm{m}^{2}$

Figure 3-11. Levelized Annual Energy Cost as a Function of the Hemispherical Reflectivity for a $75-\mathrm{NW}$ Plant with $750^{\circ} \mathrm{C}$ Delivery Temperature and Two Heliostat Costs. The LEC for the baseline glass/metal heliostat system is included for comparison.

Figure 3-1 for the two assumed stretched membrane heliostat costs of $\$ 50 / \mathrm{m}^{2}$ and $\$ 100 / \mathrm{m}^{2}$. As might be expected, the decrease in delivered energy is nearly proportional to the decrease in hemispherical reflectivity.

In the base case the hemispherical reflectivity assumed is the same as that of the glass/metal heliostat. However, it may be that environmental degradation of the polymer surface due to ultraviolet radiation, temperature cycles, moisture, and dirt will be more severe than that of a glass/metal surface. The results in Figures $3-10$ and $3-11$ illustrate the sensitivity to such degradation.

The sensitivity of the annual energy delivery and the LEC to changes in the surface normal, specularity, and reflectivity parameters is shown in Figures 3-12 and 3-13 for two delivery temperatures. Both figures show the results for a $75-\mathrm{MW}$ system using $100-\mathrm{m}^{2}$ focused stretched membrane heliostats. Figure 3-12 shows the sensitivity of the energy delivered per unit area of heliogtat (E/A), and Figure 3-13 shows the sensitivity of the LEC assuming $\$ 50 / \mathrm{m}^{2}$ heliostat costs. Clearly, the biggest effect is with variations in hemispherical reflectivity, and the next largest effect is caused by surface normal effects. It is seen that receiver temperature causes only moderate effects. Noting the scale on the ordinate of these plots shows that, in fact, rather dramatic changes in $\sigma_{d}$ and $\sigma_{\Psi}$ have only small $(<10 \%)$ changes in $E / A$, LEC, and the energy delivered per unit of heliostat area. 


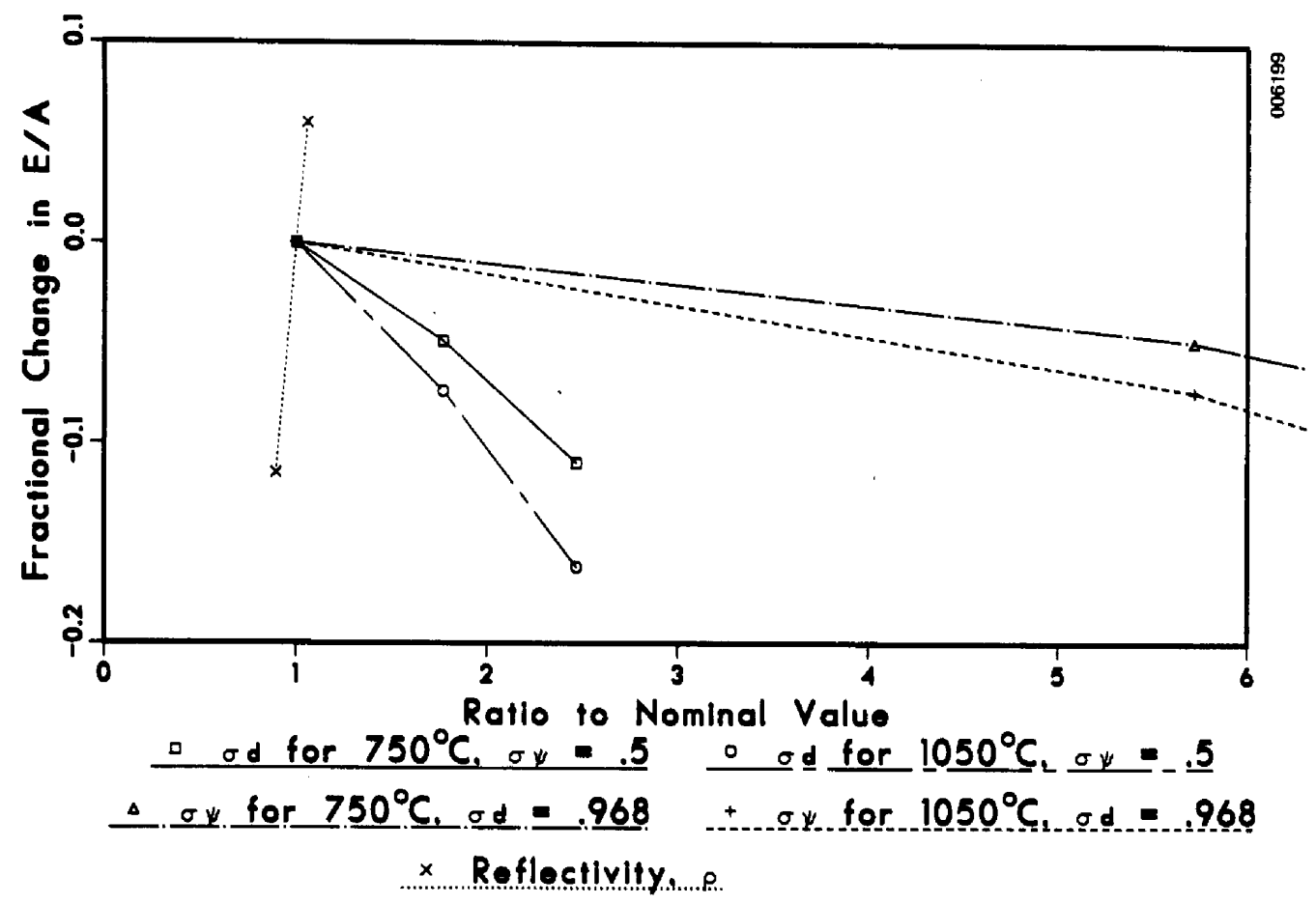

Rigure 3-12. A Comparison of the Relative Bffects of Several Optical Parameters on the Annual Energy Delivery for a 75-Mw Plant Using Focused Stretched Membrane Heliostats. The ordinate shows the fractional change in the energy delivered relative to the baseline, while the abscissa represents the ratio of the independent parameter to its baseline value. The independent parameters are the surface normal errors, $\sigma_{d}$ (assuming $\sigma_{y}=0.5 \mathrm{mrad}$ ), the specularity errors, $\sigma_{\psi}$ (assuming $\sigma_{d}=0.968 \mathrm{mrad}$ ), and the hemispherical reflectivity, $\rho$.

\subsection{IMPACT OP OPTICAL ERROR UNCERTAIMTIES}

The results presented in Section 3.2 for focused stretched membrane heliostats and in Section 4.2 for unfocused stretched membrane heliostats presume that the optical error as represented by $\sigma_{\text {opt }}$ is accurately estimated. To derive these results the energy delivered by the system was calculated as a function of $\sigma_{o p t}$ only after the system configuration--field layout, receiver aperture size, etc.-had been optimized at the assumed error level oopt. If the optical errors have been misestimated or if they change over time, it is possible that the energy delivered by the system will vary considerably from the results in Sections 3.2 and 4.2 .

To investigate the sensitivity of the delivered energy to the uncertainty in the optical error estimates (i.e., to $\sigma_{o p t}$ ), we conducted a 1 imited number of simulations in which the system configuration was optimized at one optical error level and performance was estimated at a second error level. The results of these simulations are illustrated in Figure 3-14, where for a 75-MW plant delivering thermal energy at $450^{\circ} \mathrm{C}$ we show the sensitivity of system performance to optical errors for several optimization assumptions. The uppermost curve corresponds to the approach used in all of the other analyses 


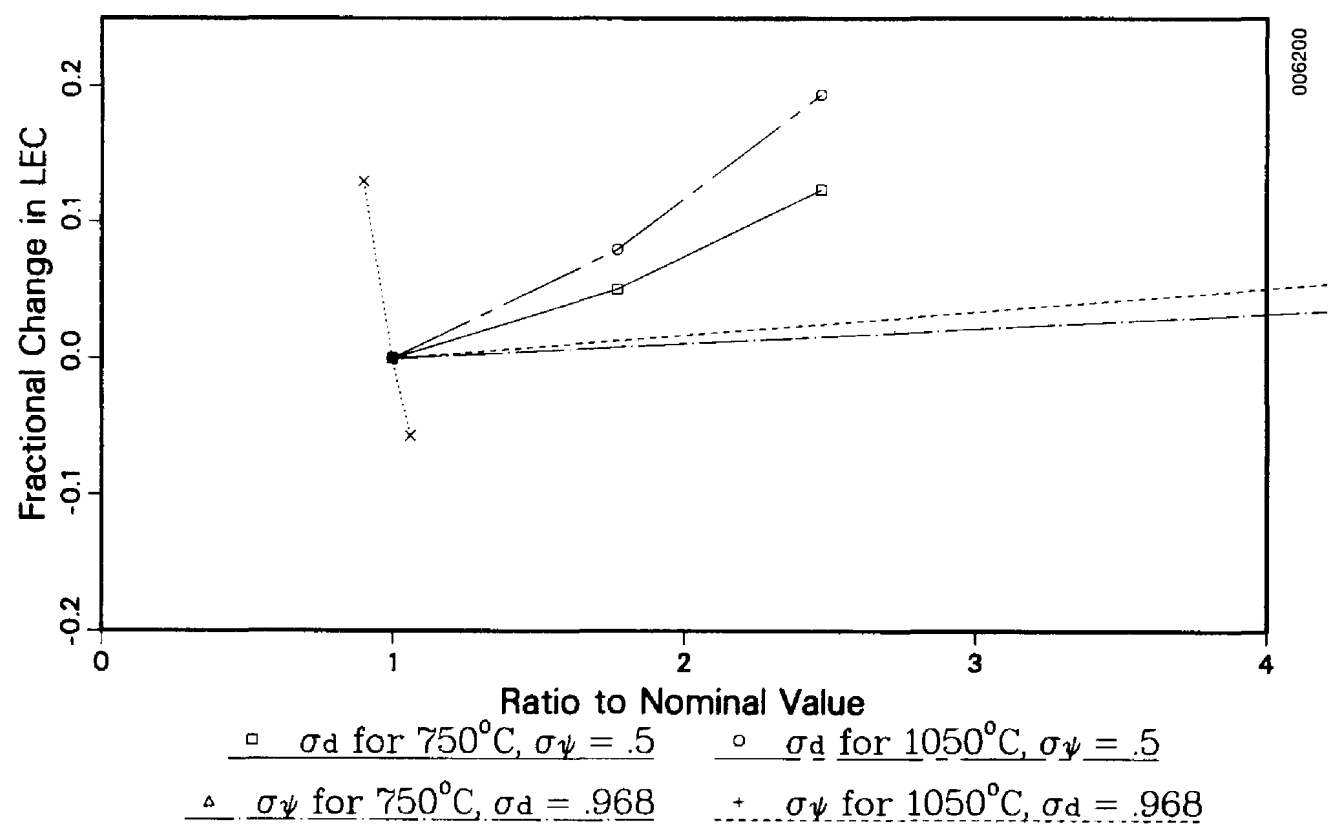

$\times$ Reflectivity, $\rho$

Figure 3-13. A Comparison of the Relative Effects of Several Optical Parameters on the Levelized Energy Cost for a 75-MW Plant Using $\$ 50 / \mathrm{m}^{2}$ Focused stretched Membrane Heliostats. The ordinate shows the fractional change in the LEC relative to the baseline, while the abscissa represents the ratio of the independent parameter to its baseline value. The independent parameters are the surface normal errors, $\sigma_{d}$ (assuming $\sigma_{y}=0.5 \mathrm{mrad}$ ), the specularity errors, $\sigma_{w}$ (assuming $\sigma_{d}=0.968 \mathrm{mrad}$ ), and the hemispherical reflectivity, $p$.

presented in this work (i.e., the system is performance optimized for each specific known error). The second curve, which is coincident with the first curve at a value of $\sigma_{0 p t}=4.8 \mathrm{mrad}$, corresponds to a system configuration that has been optimized for an optical error of $\sigma_{\text {opt }}=4.8 \mathrm{mrad}$, after which the performance was evaluated at lower optical errors. The third curve, which has the steepest slope and is coincident with the upper curve at $\sigma_{\text {opt }}=2$, corresponds to a system configuration that has been optimized for $\sigma_{o p t}=2 \mathrm{mrad}$. This same configuration is then held constant while the actual optical error is increased and system performance determined.

Several conclusions can be drawn from this figure. As expected, the system with the best performance is that which considers the field/receiver configuration to be performance optimized at each optical error level (first curve). The performance sensitivity to optical surface errors is greatest for systems optimized for too low an error estimate (third curve); the least sensitivity is seen to occur for the system optimized for too high an error (second curve). Thus the penalty for assuming too small an error is in general significantly greater than for assuming too large an error. Hence from a designer's perspective it is better to design for a larger error in the face 


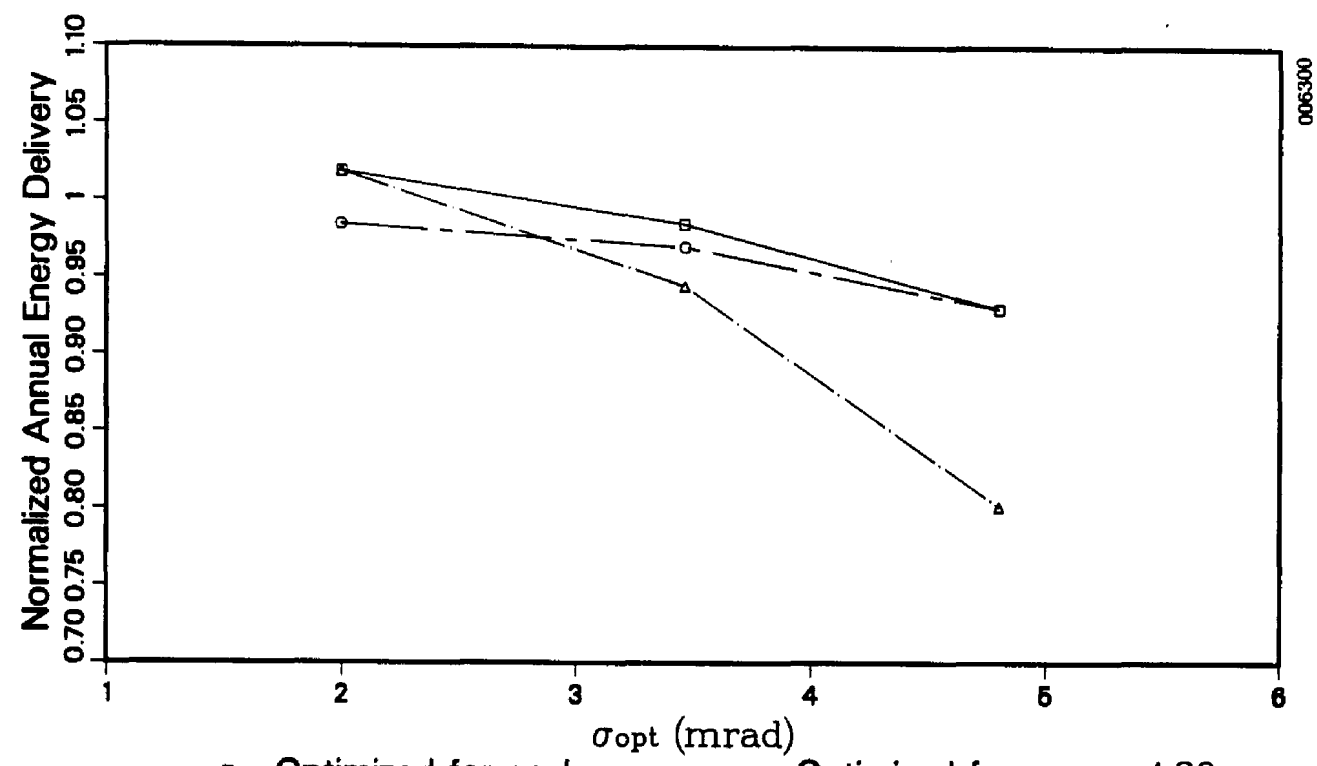

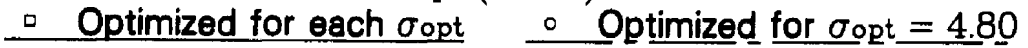

$\triangle$ Optimized for oopt $=2.00$

Figure 3-14. Normalized Annual Delivered Energy vs. Oopt Corresponding to Three Optimization Procedures. The deta are for a $75-\mathrm{MW}_{\mathrm{th}}$ plant at $450^{\circ} \mathrm{C}$ using focused stretched membrane heliostats.

of uncertainty than too small an error. The greater sensitivity to underestimation of the optical errors in the system optimization is due to the precipitous increase in spillage losses that occurs when the receiver aperture is undersized. 
SECTION 4.0

\author{
SYSTEMS ANALYSIS OF UNFOCUSED STRETCHED MEMBRANE HELIOSTATS
}

Unfocused stretched membrane heliostats may have the potential advantage relative to focused stretched membrane heliostats because of lower initial and operating costs since no control mechanism or positive pumping is required to maintain the focus. However, they also have the disadvantage of lower performance relative to a focused module of the same size. This lower performance accrues for two reasons. First, since no focusing is assumed, the reflected image size will be a strong function of the reflector size. Second, since no control of the surface is assumed, the wind and weight loads will cause the surface to deform relative to its initial state considerably more than with the focused modules, which have pressure-controlled reflective surfaces.

In this section we examine these cost/performance tradeoffs by comparing the overall cost and performance of systems employing focused and unfocused stretched membrane heliostats. Cost and performance of the two types of systems will be compared for a range of system delivery temperatures $\left(450^{\circ}, 750^{\circ}\right.$, $\left.1050^{\circ} \mathrm{C}\right)$, plant sizes $\left(75 \mathrm{MW}_{t h}, 450 \mathrm{MW} / \mathrm{h}\right)$, heliostat sizes $\left(25-100 \mathrm{~m}^{2}\right)$, and heliostat surface errors ( $\sigma_{o p t}=2$ to $\left.3.5 \mathrm{mrad}\right)$. As in section 3.0 , the energy delivered is always presented in terms of the annual energy delivered to the base of the tower per square meter of heliostat area.

\title{
4.1 ASSUMPTIONS
}

The unfocused stretched membrane modules are assumed to be flat in their initial state. Surface errors from manufacturing and from wind- and weightinduced loading are measured relative to that initially perfect flat state. The assumptions used in the evaluation of systems with unfocused stretched membrane heliostats are otherwise the same (with one exception) as those presented in section 3.0 for focused heliostats. The only exception is that the surface errors for the unfocused units are assumed to include axisymmetric deformations, as described in Section 2.0 and Appendix B. This axisymmetric deformation increases the standard deviation of the surface errors as measured in a single dimension from $\sigma_{o p t}=2.0 \mathrm{mrad}$ to $\sigma_{o p t}=3.46 \mathrm{mrad}$ for the base case.

$\sigma_{o p t}=2.0 \mathrm{mrad}$ represents a lower bound on the optical surface error attainable with unfocused and uncontrolled reflector surfaces. This level is most likely to be approached by the small diameter designs, since the major contributor to values above $\sigma_{o p t}=2.0 \mathrm{mrad}$ results from axisymmetric deformation, which is proportional to the radius of the design (assuming all other variables are constant). On the other hand, since we have taken no credit for gravity induced focusing, by careful design one may be able to do noticeably better than $\sigma_{\text {opt }}=3.46 \mathrm{mrad}$ even for the large designs. 


\subsection{RESULTS FOR UNFOCUSED STRETCHED MEMBRANE SYSTEMS}

Figure 4-1 presents a performance comparison of the three heliostat types considered--glass/metal, focused stretched membrane, and unfocused stretched membrane--for the base-case assumptions and delivered energy temperatures from $450^{\circ}$ to $1050^{\circ} \mathrm{C}$ and for a $75-\mathrm{MW}$ th plant size. The top two curves representing the focused stretched membrane and glass/metal heliostats present the same results shown in Figure 3-1. Although the base-case assumptions for the focused and unfocused results of Figure 4-1 are the same except for the surface errors, the system configuration has been reoptimized for each case based on performance for each heliostat type. The bottom curve representing the unfocused case indicates that the higher surface errors $\left(\sigma_{0 p}=3.46\right)$ assumed for this case together with the size effect for the $100-\mathrm{m}^{2}$ flat unfocused heliostats reduce the delivered energy by $15 \%$ to $30 \%$ relative to the focused case. The greatest reduction occurs at the higher delivered energy temperatures because the receiver aperture is smaller and focusing more important.

In Figure 4-2 the annual energy delivered per square meter by a 75-MW unfocused stretched membrane heliostat system has been normalized by the annual energy delivered from a system using $100-\mathrm{m}^{2}$ focused stretched membrane heliostats with $\sigma_{\text {opt }}=2.0 \mathrm{mrad}$. The energy delivered by the unfocused systems more closely approaches that from a focused system (the ratio approaches 1.0 )

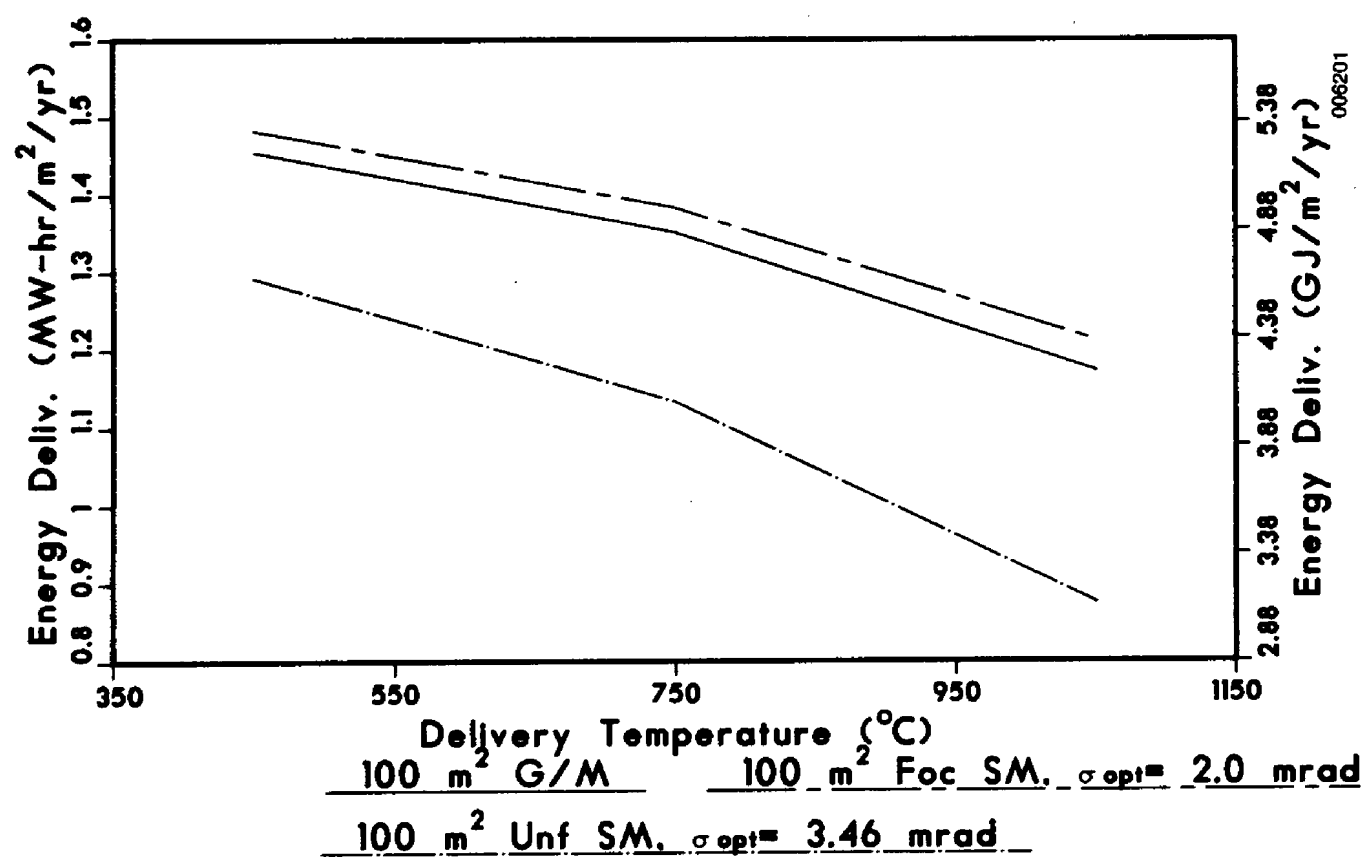

Figure 4-1. Comparison of the Baseline Cases for Glass/Metal and Focused and Unfocused Stretched Membrane Heliostats. The total surface errors, opt, are $2.00 \mathrm{mrad}$ for the $81 \mathrm{ass} / \mathrm{metal}$ and the focused stretched membrane heliostats, and $3.46 \mathrm{mrad}$ for the unfocused stretched membrane heliostats. 


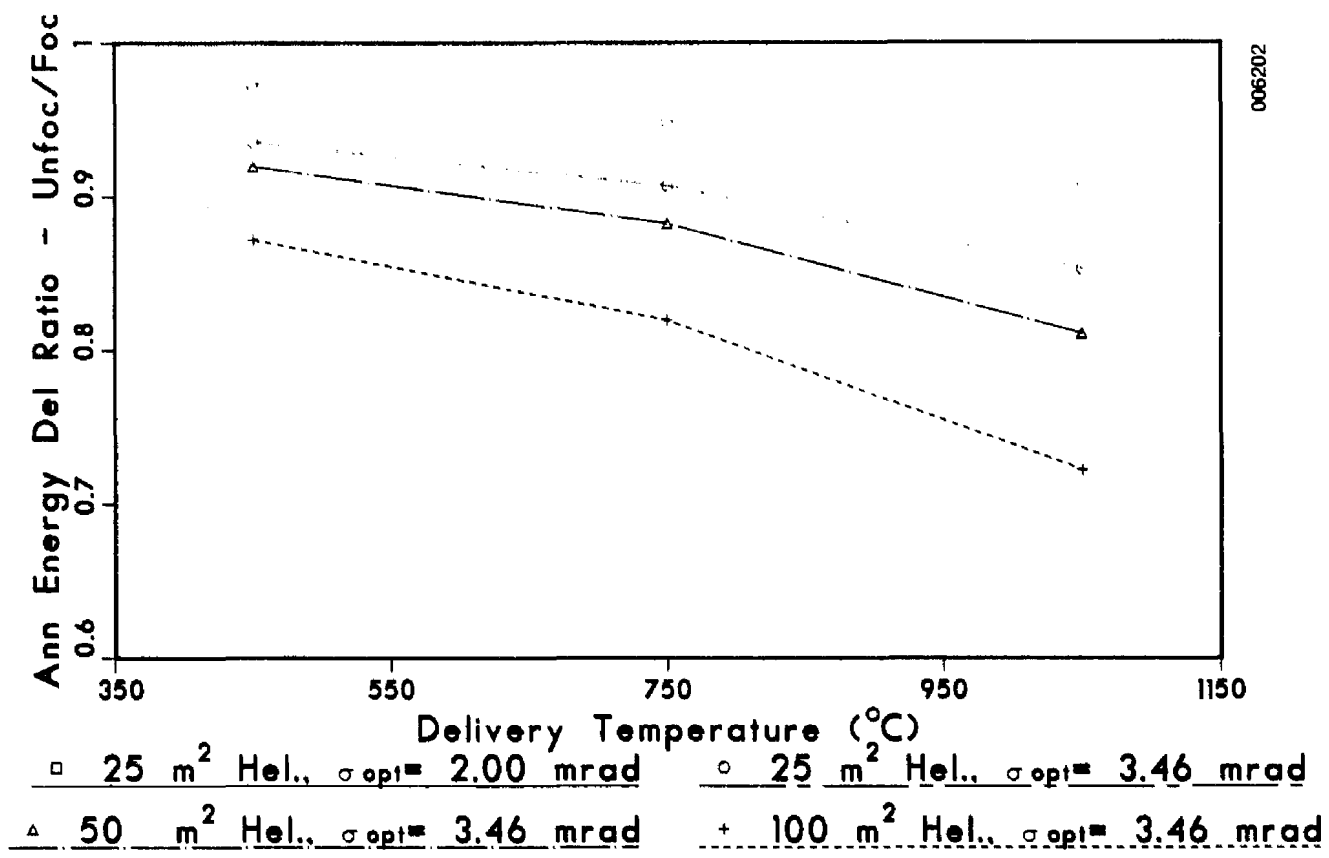

Figure 4-2. Ratio of Annual Energy Delivery for the Unfocused Stretched Membrane Heliostats to That for the Focused Stretched Membrane Heliostat as a Function of Delivery Temperature for 3 Heliostat Sizes and a 75-MW Plant. The total surface errors, opt, of 3.46 mrad represent the baseline for the unfocused gystems, while the curve for $\sigma_{0 p t}$ of $2.00 \mathrm{mrad}$ is an upper bound on the reduction in surface errors possible with smaller heliostat sizes.

as the size of the unfocused modules is decreased. This illustrates that as the area (diameter) of an unfocused stretched membrane heliostat is reduced the losses caused by lack of focusing (heliostat size errors) are reduced.

Note that the delivery temperature also has a significanf effect on the relative performance. At lower temperatures even the $100-\mathrm{m}^{2}$ unfocused stretched membrane heliostats can produce about $88 \%$ as much energy per unit area as the focused modules. This is because the lower temperature systems have relatively larger apertures and lower thermal losses per unit area of aperture. Therefore, the fractional increase in the optimized aperture area is smaller, and the penalty for increasing the aperture size is also smaller.

Figure 4-3 shows that the heliostat size effect is also much less influential for larger plant sizes. The reason for this difference is that, as with the lower temperature systems, the larger plants have intrinsically larger apertures, and the fractional increase in the required optimum aperture size is accordingly smaller.

The bottom three curves in Figures 4-2 and 4-3 are all for a constant value of $\sigma_{\text {opt }}(=3.46 \mathrm{mrad})$. This is equivalent to assuming that a decrease in the hellostat size affects only the size error (the error introduced by the finite 


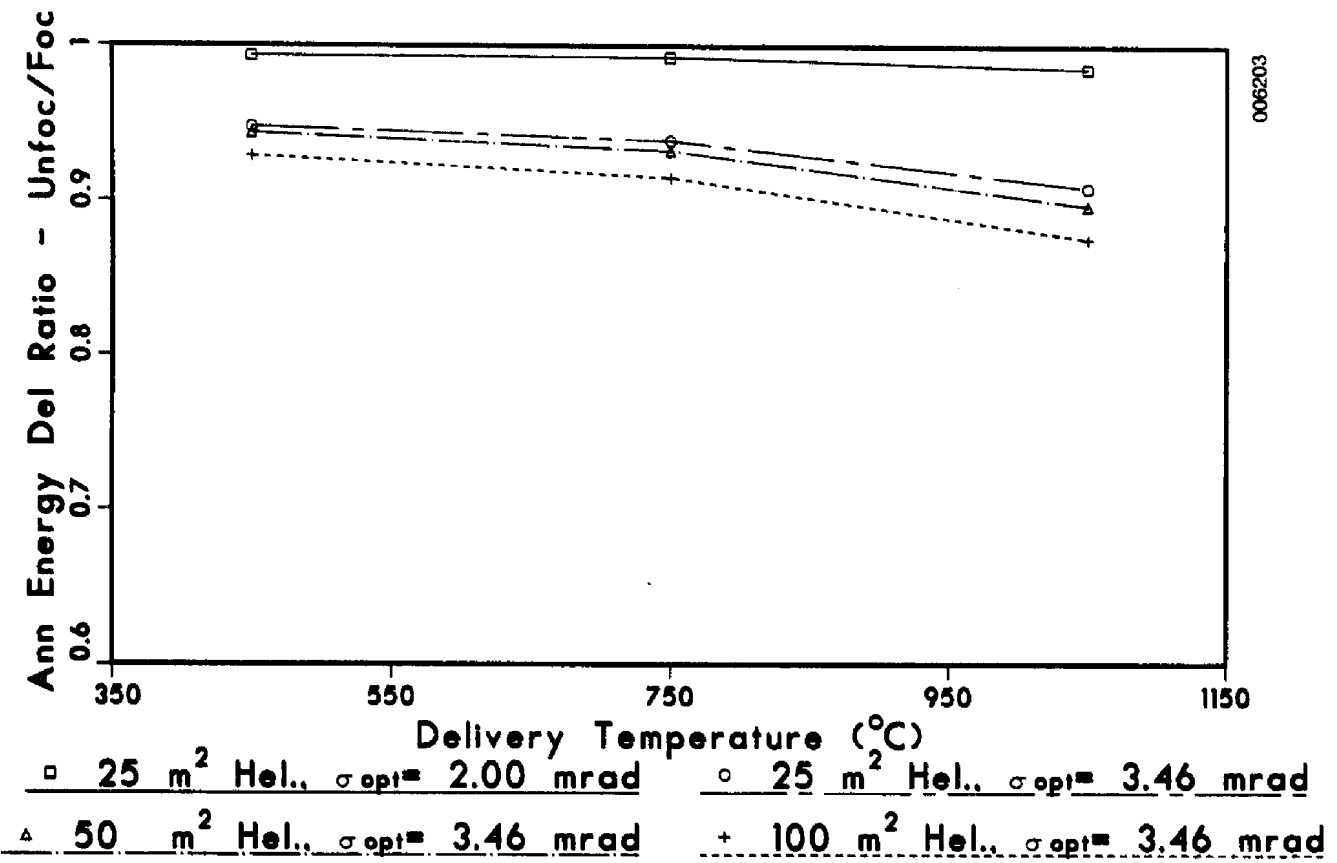

Rigure 4-3. Ratio of Annual Bnergy Delivery for the Unfocused Stretched Membrane Heliostat to That for the Focused Stretched Membrane Beliostat as a Punction of Delivery Temperature for 3 Heliostat Sizes and a 450-MW Plant. The total surface errors, $\sigma_{\text {opt }}$, of $3.46 \mathrm{mrad}$ represent the baseline for the unfocused $3 y$ stems, while the curve for $\sigma_{0 p t}$ of $2.00 \mathrm{mrad}$ is an upper bound on the reduction in surface errors possible with smaller heliostat sizes.

size of the unfocused heliostat) and not the surface errors. In fact, as described in Section 2.0, the surface errors will also probably decrease with decreasing heliostat size. The top curve in both figures presents a likely upper bound on the performance improvement possible with smaller unfocused heliostats, since it incorporates a reduction of the surface errors to the same level as that assumed for the base-case focused heliostats $\left(\sigma_{o p t}=2.00 \mathrm{mrad}\right)$. Note that in this case the decrease in performance for the $25-\mathrm{m}^{2}$ unfocused heliostats relative to the focused heliostats is only about $3 \%$ for the $75-M W$ system operating at $450^{\circ} \mathrm{C}$, and less than $1 \%$ for the 450-MW system. Thus, if these errors could be achieved, the performance of smaller unfocused heliostats could effectively equal that of the state-of-theart glass/metal heliostats.

The results presented in Figures 4-2 and 4-3 are transformed into a comparison of the relative economics of focused-versus-unfocused stretched membrane heliostats in Figures 4-4 and 4-5 (i.e., how much more one could afford to pay for focused heliostats without increasing the levelized cost of delivered energy). The unfocused heliostats were assumed to cost $\$ 50 / \mathrm{m}^{2}$, and the economic assumptions were taken from the current Multiyear Program Plan [14]. In essence, Figures 4-4 and 4-5 show that, depending on the situation, unfocused heliostats begin to be advantageous if the incremental cost of the focusing 


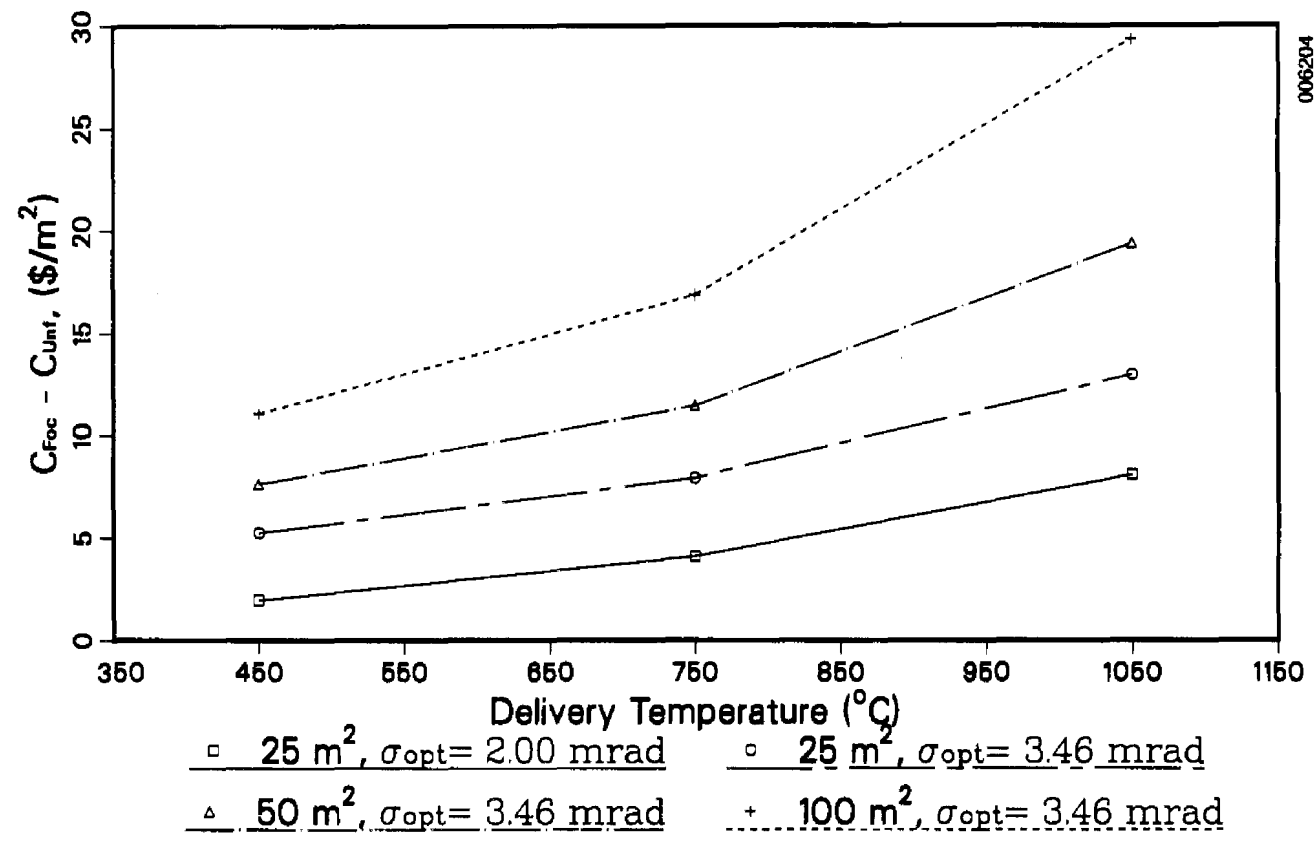

Figure 4-4. Allowable Cost of Focusing as a Runction of Delivery Temperature for a 75-MW Plant at Several Heliostat Sizes and Total Surface Errors. The allowable cost of focusing is the increase in the cost of the heliostats (per $\mathrm{m}^{2}$ ) you could afford for focusing without increasing the levelized energy cost. These values are based on a constant unfocused heliostat cost of $\$ 50 / \mathrm{m}^{2}$.

(above the cost of comparable unfocused heliostat) exceeds about $\$ 4-\$ 5 / \mathrm{m}^{2}$ for the cases with the larger surface errors $\left(\sigma_{o p t}=3.46\right)$. However, if the surface errors on the unfocused heliostats can be reduced to those of the focuged module $\left(\sigma_{0 p}=2\right.$ ) the allowable cost of focusing drops to less than $\$ 1 / \mathrm{m}^{2}$ for the $458 \mathrm{PWW}$ plant. As with the earlier results, the figures support the conclusion that the unfocused heliostats appear to be advantageous for larger plants, lower temperatures, and for smaller heliostat sizes.

One assumption implicit in the analysis behind Figures 4-4 and 4-5 is that heliostat cost per square meter is independent of heliostat size. Clearly this assumption ignores the increase in the number of supports, tracking mechanisms, and controls that accompany a decrease in the size of the heliostat (and a corresponding increase in the number of heliostats).*

*Additionally, it could be argued that some extra controls would be required for emergency defocusing of the unfocused heliostats. This is because in an emergency (e.g., $108 \mathrm{~s}$ of fluid in the absorber) the beam from the focused heliostats can be quickly dispersed merely by eliminating the vacuum (or even pressurizing) in the space between the membranes. However, with the unfocused stretched membrane heliostats (as with the glass/metal heliostats currently in use) the heliostats must actually move off their aim point in order to defocus the field. 


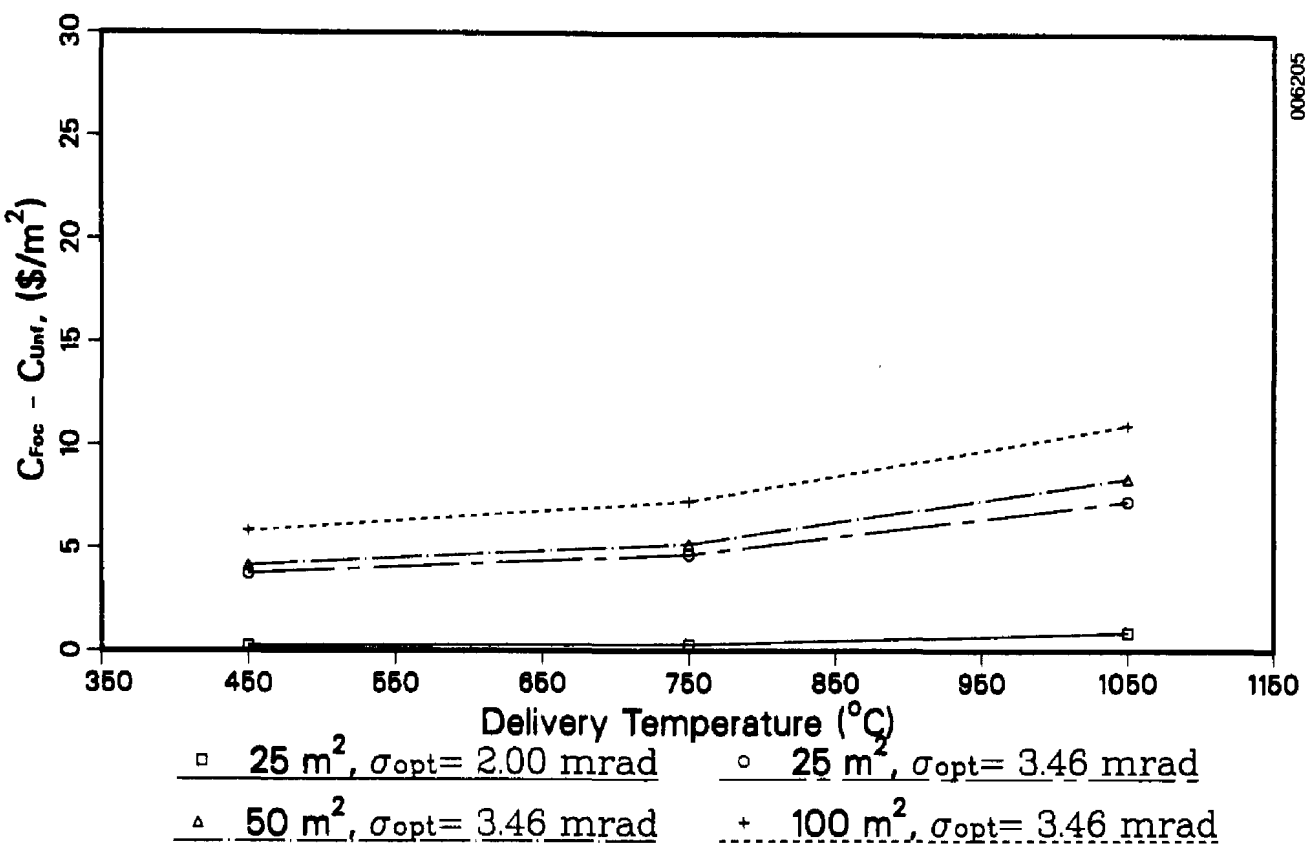

Pigure 4-5. Allowable Cost of Focusing as a Function of Delivery Temperature for a 450-MW Plant at Several Heliostat Sizes and Total Surface Errors. The allowable cost of focusing is the increase in the cost of the heliostats (per $\mathrm{m}^{2}$ ) you could afford for focusing without increasing the levelized energy cost. These values are based on a constant unfocused heliostat cost of $\$ 50 / \mathrm{m}^{2}$.

Nonetheless, the results presented should be useful as guidelines as long as the total ingtalled cost per square meter is used in the comparison.

Although the size error introduced by unfocused stretched membrane heliostats can be large relative to the surface normal and specularity errors, the performance of a system with unfocused heliostats is essentially just as sensitive to surface normal and specularity errors as a system with focused heliostats. Figures $4-6$ and 4-7 compare the decrease in performance for these two types of heliostats as a function of the combined surface normal and specularity errors ( $\sigma_{o p t}$ ) for several sizes of unfocused heliostats.

The results for the $75-M W$ system in Figure $4-6$ show that the difference in heliostat size errors nearly overwhelms any difference caused by the surface errors. However, in Figure 4-7 the results for the 450-MW system indicate that the two error sources cause about the same magnitude of decrease in performance for the ranges studied.

The results presented here indicate that smaller plant sizes are significantly more sensitive to decreases in the optical quality of the reflected beam (caused by either surface errors or size effects) than larger plants. To some extent, this increase in sensitivity is exaggerated because the receiver used in all of the cases presented in Sections 3.0 and 4.0 was a general cavity 


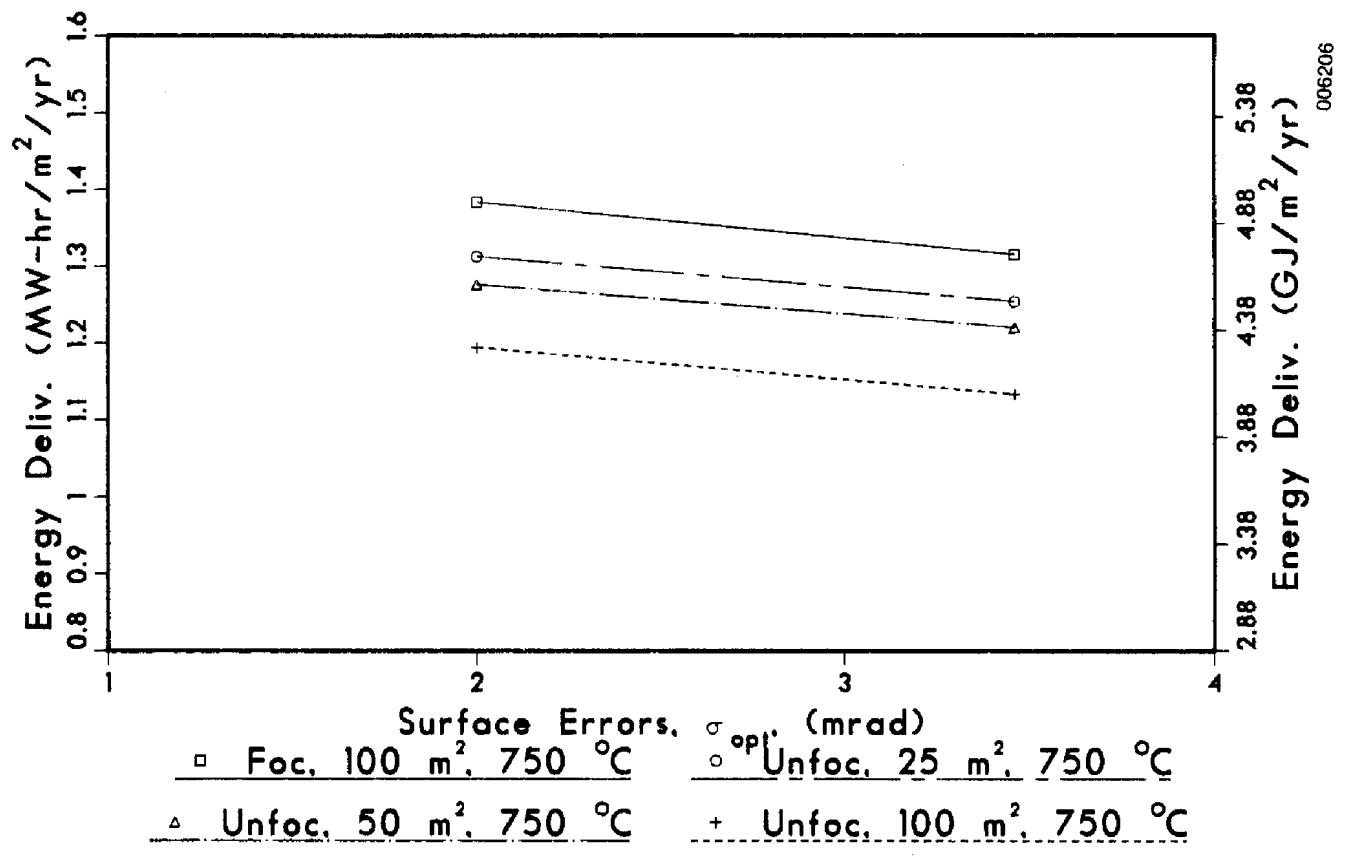

Figure 4-6. Effect of Surface Errors on the Annual Energy Delivery for Several Sizes of Unfocused Stretched Membrane Heliostats in a 75-MW Plant at $750^{\circ} \mathrm{C}$ Delivery Temperature. The effect of the surface errors on focused stretched membrane heliostats is included for comparison.

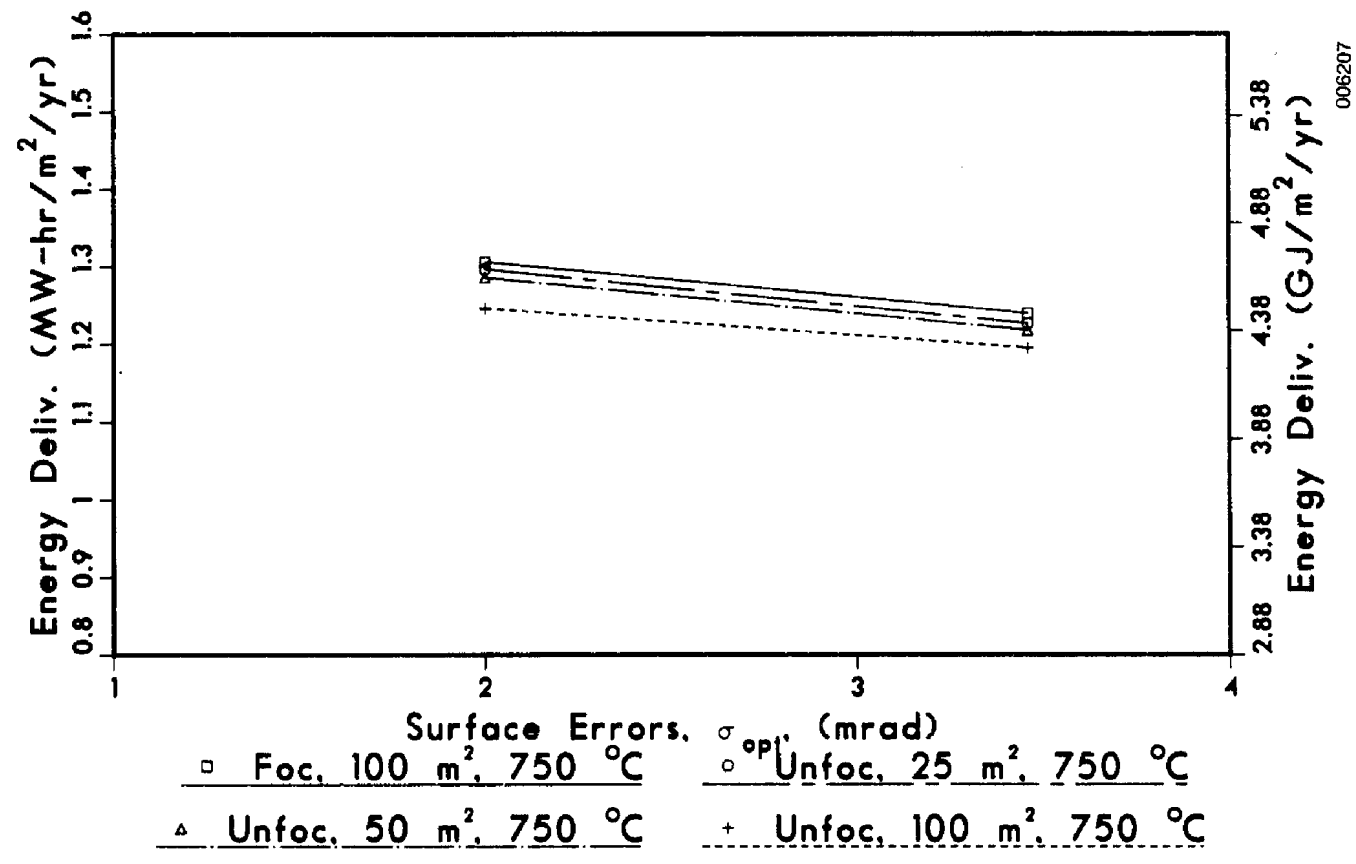

Figure 4-7. Effect of Surface Errors on the Annual Energy Delivery for Several Sizes of Unfocused Stretched Membrane Heliostats in a 450-MW Plant at $750^{\circ} \mathrm{C}$ Delivery Temperature. The effect of the surface errors on focused stretched membrane heliostats is included for comparison. 
configuration originally designed for high temperatures and relatively high absorber plane flux levels.

Unfortunately, a single receiver configuration cannot cover the entire range of potential applications. In this case, the particular geometry chosen could not simultaneously accommodate both the desired absorber flux levels and the large apertures required to optimize the unfocused, poor optical quality cases. Because of this limitation, another receiver design was briefly investigated that outperformed the base-case receiver for these cases. The geometry of this receiver and an example of the potential improvements in performance are given in Appendix F.

Even with this exaggerated decrease in performance at the smaller plant size, the unfocused stretched membrane heliostats demonstrate a remarkable level of performance, competing well with both the focused stretched membrane heliostats and the glass/metal heliostats in a significant number of cases. The choice of focused-versus-unfocused designs for a system designer will clearly depend strongly on the particular application. In terms of the Solar Thermal Program, the focused stretched membrane heliostats show a promise for applicability over a broader range of applications, and would probably be favored on that basis. 
SECTION 5.0

\section{CONCLUSIONS}

In these systems comparisons of stretched membrane heliostats with glass/metal concepts we have investigated a wide range of system and component parameters, including variations in system operating temperature, system size, heliostat size, macroscopic surface normal errors, specularity, and hemispherical reflectivity. Considering the range of temperatures and system sizes investigated, the current study provides information on a larger range of sensitivity variations than any other previous documented study of heliostats, whether glass/metal or stretched membrane.

In support of the systems studies, the major findings from recent and ongoing structural and optical response investigations of individual stretched membrane modules are cast in terms of performance parameters needed to describe collector field performance. As such, the current study integrates a large number of results and definitions from systems, structural, and optical materials studies into a single unified presentation.

Based on the range of parameters described above, it appears that focused stretched membrane heliostats may have performance levels that are quite close to and possibly slightly better than the corresponding glass/metal heliostats. This is due to their apparent ability to maintain good macroscopic surface quality under anticipated wind and weight induced loading and their round shape, which leads to reduced blocking and shading and tighter packing when compared to the rectangular shape of glass/metal heliostats. Further, there does not appear to be any particular performance advantage of one concept over the other when variations in either temperature or system size are considered.

Wind- and weight-induced surface errors on the focused stretched membrane heliostats appear to be potentially quite small if the vacuum/pressure control on the reflector surface, which eliminates the axisymmetric membrane deformation, works effectively. In fact, the macroscopic surface qualities of the stretched membrane modules appear to be quite close to (and maybe slightly better than) those corresponding to glass/metal heliostats under the anticipated wind- and weight-loading environments. We have not directly evaluated cases where the surface control is not totally effective, which might occur when the nonuniform pressure loading on the reflective surface is considered. Further, good macroscopic surface quality also assumes that the manufacturing errors can be controlled to reasonable levels that are also on the order of those currently found in glass/metal heliostats. This is a major assumption that is being evaluated in the current development program by SNLL.

When the stretched membrane heliostat is focused, module size does not appear to be an important parameter with respect to performance. Thus the cost associated with other design parameters and subelements will dictate the appropriate size to consider. This was found to be true over the range of system sizes and delivery temperatures considered in this study.

Glass/metal mirrors typically have a high degree of specularity, with $90 \%$ of the energy reflected in a half-cone angle, $\Psi$, of about $1.0 \mathrm{mrad}$. With the 
advent of polymeric coatings for reflective surfaces, the surface specularity has become an issue for concern. The results presented here do not give an explicit limit on the allowable specularity errors. Rather, they demonstrate the effect of an increase in the overall surface errors, which also include the effects of surface normal errors and other surface phenomena that affect the size of the reflected beam.

Within this context, we can say that if the other surface errors are roughly the same for the stretched membrane heliostats as for the glass/metal (i.e., $\left.\sigma_{d}=0.968 \mathrm{mrad}\right)$, then even if the specularity half-cone angle, $\Psi$, that includes $90 \%$ of the reflected energy were to increase to $6 \mathrm{mrad}$, the performance would fall off only about $5 \%$ for the $75-\mathrm{MW}_{\mathrm{th}}$ plant size, and even less for larger plants. Since the range from 1 to 6 mrad encompasses the range currently being reported for polymer coated reflective surfaces [16], it appears that specularity effects will probably not be a major issue in the acceptance of stretched membrane heliostats.

For focused heliostat modules the biggest impact on performance appears to be potential variations in hemispherical reflectivity (similar sensitivity would be seen with the glass/metal concepts). The next largest impact on performance appears to be due to surface normal errors and then to specularity. In fact, nothing in this study has indicated sensitivities that would be different from those anticipated for glass/metal heliostats. However, this is not the case for unfocused stretched membrane heliostats.

With unfocused modules, heliostat size effects can have a significant impact on system performance. This is because for large modules the effective reflected flux cylinder size for the module is directly proportional to module size. In many cases the diameter of the reflected flux cylinder due to heliostat size can be as large as the other reflective beam broadening effects such as the finite sun size.

In general, the benefit of focusing increases with increases in module size and system operating temperature, but decreases with plant size. For instance, for a $75-\mathrm{MW}_{\text {th }}$ plant size, the $100-\mathrm{m}^{2}$ unfocused heliostats deliver $13 \%$ less energy at $450^{\circ} \mathrm{C}$ and about $28 \%$ less energy at $1050^{\circ} \mathrm{C}$ when compared to the focused heliostats. For a 450-MW plant the corregponding numbers are $8 \%$ and $13 \%$ respectively. On the other hand, the $25-\mathrm{m}^{2}$ unfocused heliostats deliver only $6 \%$ less energy at $450^{\circ} \mathrm{C}\left(13 \%\right.$ less at $\left.1050^{\circ} \mathrm{C}\right)$ for the $75-\mathrm{MW}_{\mathrm{th}}$ plant.

It appears to be prudent to pursue the focused designs in the near term, to give these collectors the widest range of applicabilities (field size and application temperature) in the limited number of systems to be built. However, in the long term this decision should be reviewed if the future applications focus on low-temperature IPH systems, large plant sizes, coupling to external receivers, or small heliostat sizes. 
SECTION 6.0

REPERENCES

1. Murphy, L. M., Technical and Cost Benefits of Lightweight, StretchedMembrane Heliostats, SERI/TR-253-1818, Golden, CO: Solar Energy Research Institute, May 1983 .

2. Edlestein, R. B., Solar Thermal Cost Goals, SERI/TP-633-1063, Golden, CO: Solar Energy Research Institute, January 1981.

3. Mavis, C. L., et a1., A Description and Assessment of Solar Central Receiver Systems Technology, SAND 82-8023, Livermore, CA: Sandia National Laboratories, forthcoming.

4. Murphy, L. M., and D. V. Sallis, Analytical Modeling and Structural Response of a Stretched Membrane Reflective Module, SERI/TR-253-2101, Golden, CO: Solar Energy Research Institute, May 1984.

5. Murphy, L. M., A Variational Approach for Predicting the Load Deformation Response of a Double Stretched Membrane Reflector Module, SERI/TR-252-2626, Golden, CO: Solar Energy Research Institute, draft, February 1985.

6. Murphy, L. M., D. V. Sallis, and D. Simms, Structural Design Considerations for Stretched Membrane Heliostat Reflector Modules with Stability and Initial Imperfection Considerations, SERI/TR-253-2338, Golden, CO: Solar Energy Research Institute, draft, May 1985.

7. Pettit, R. B., C. N. Vittitoe, and F. Biggs, "Simplified Calculational Procedure for Determining the Amount of Intercepted Sunlight in an Imaging Solar Concentrator," Journal of Solar Energy Engineering, Vol. 105, February 1983.

8. Rabl, A., "Concentrating Collectors," in Solar Energy Technology Handbook: Part A, Engineering Fundamentals, Dickenson and Chermisenoff, eds., Marcel Dekker, Inc., 1980.

9. Vittitoe, C. N., F. Biggs, and R. E. Lighthill, Helios - A Computer Program for Modeling the Solar Thermal Test Facility, SAND76-0346, June 1977; and G. M. Schrenk, Analysis of Solar Reflectors: Mathematical Theory and Methodology for Simulation of Real Reflectors, GMC-AD-EDR-3693, December 1963.

10. Dellin, T. A., et al., A User's Manual for DELsol2: A Computer Code for Calculating the Optical Performance and Optimal System Design for Solar Thermal Central Receiver Plants, SAND81-8237, Sandia National Laboratories, August 1981. 
11. Delaquil, P., and J. V. Anderson, The Performance of High-Temperature Central Receiver Systems, SAND84-8233, Sandia National Laboratories, 1984.

12. Abrams, M., RADSOLVER - A Computer Program for Calculating Spectrally Dependent Radiative Heat Transfer in Solar Cavity Receivers, SAND81-8248, Sandia National Laboratories, September 1981.

13. Emery, A. F., Instructional Manual for the Program "Shapefactor", SAND80-8027, Sandia National Laboratories, October 1980.

14. Sandia National Laboratories, National Solar Thermal Technology Program Five Year Research and Development Plan 1985-1989, Draft, Livermore, CA, December 1984. Also, personal communication from Tom Williams of Pacific Northwest Laboratories.

15. Eicker, P. J., et al., Design, Cost and Performance Comparisons of Several Solar Thermal Systems for Process Heat, SAND 79-8283, Livermore, CA: Sandia National Laboratories, Vols. I through V, April 1981.

16. Schissel, P., Solar Energy Research Institute, Materials Research Branch, personal communication.

17. Randa11, P. E., and N. R. Grandjean, Correlation of Insolation and Wind Data for SOLMET Stations, SAND82-0094, Albuquerque, NM: Sandia National Laboratories, April 1982.

18. MeDonnel1 Douglas Astronautics Co., Optimization of the Second Generation Heliostat and Specification, Contractor Report MDC9762, Sandia Report SAND82-8181, prepared for Sandia National Laboratories, Livermore, CA, May 1982.

19. Schaeffer, H. G., MSC/NASTRAN PRIMER - Static and Normal Mode Analysis: A Study of Computerized Technology, Mount Vernon, NH: Schaeffer Analysis, Inc., 1979.

20. Kraabel, J. S., and D. L. Siebers, Estimating Convective Energy Losses from Solar Central Receivers, SAND84-8717, Sandia National Laboratories, April 1984.

21. Battleson, K. W., Solar Power Tower Design Guide: Solar Thermal Central Receiver Power systems, a Source of Electricity and/or Process Heat, SAND81-8005, Sandia National Laboratories, April 1981.

22. Pettit, R. B., and E. P. Roth, "Solar Mirror Materials: Their Properties and Uses in Solar Concentrating Collectors," in Solar Materials Science, Academic Press, 1980.

23. Pettit, R. B., "Characterization of the Reflected Beam Profile of Solar Mirror Materials," Solar Energy, Vol. 19, 1977, p. 733.

24. Biggs, F., and C. N. Vittitoe, The Helios Model for the Optical Behavior of Reflecting Solar Concentrators, SAND76-0347, Sandia National Laboratories, March 1979. 


\section{APPEADIX A}

\section{RELATING PROBABILISTIC AND DETERMIMISTIC SURFACE ERRORS}

Optical scattering from a reflecting solar concentrator results from specularity imperfections in the mirror material, deformation of the mirror surface, tracking errors, and the concentrator size cone. In many analyses these scattering effects are assumed to be probabilistic in nature and well approximated in a single dimension by normal probability distributions [7] with standard deviations $\sigma_{y}, \sigma_{d}, \sigma_{t}$, and $\sigma_{s}$, respectively. As such the sum of these effects can also be represented as a normal probability distribution with standard deviation $\sigma_{\text {refl }}$ calculated as shown*:

$$
\sigma_{\text {ref } 1}=\left[\sigma_{\Psi}{ }^{2}+\left(2 \sigma_{d}\right)^{2}+\left(2 \sigma_{t}\right)^{2}+\sigma_{s}{ }^{2}\right]^{1 / 2} \text {. }
$$

For the stretched membrane heliostat, Eq. A-1 [6] provides a deterministic expression for the deformation of the surface in terms of the polar radial $(r)$ and circumferential $(\theta)$ coordinates:

$$
\begin{aligned}
w(r, \theta) & =a_{0}\left[1-\left(\frac{r}{a}\right)^{2}\right]_{j}+\sum_{k=1}^{m} a_{k}\left[1-\left(\frac{r}{a}\right)^{\frac{2 \pi k}{P}} \cos \frac{2 \pi k \theta}{P}\right] \\
& +\ell_{k} \sum_{0}^{m} b_{k}\left(\frac{r}{a}\right)^{\frac{2 \pi k}{P}} \cos \frac{2 \pi k \theta}{P} .
\end{aligned}
$$

This expression corresponds to a circular membrane of radius a mounted on a frame that is supported at evenly spaced circumferential angular intervals of $P$ radians. As shown in Figure $A-1$, the norm of the gradient of this deformation provides an estimate of the surface normal error, $\phi$, due to the deformation. By integrating the norm of the gradient over the surface of the stretched membrane, a deterministic distribution of the surface normal error can be computed. Because the convolution of the deterministic distribution from surface deformation with the other three sources of error can be extremely cumbersome, it may be advantageous to continue to approximate this distribution with a normally distributed random variable. This appendix investigates the validity of such an approximation.

Earlier analysis [4] has shown that the first term $(k=1)$ in the summations of Eq. A-1 can be used by itself to provide a reasonably good estimate of the surface deformation within a few percent. Hence for this evaluation we omit terms with $k>1$ and express Eq. A-1 more simply as

$$
w(r, \theta)=b\left(\frac{r}{a}\right)^{2}+c\left(\frac{r}{a}\right)^{n} \cos n \theta+d,
$$

*The factor of two has been included for the surface deformation and tracking errors as these errors are essentially doubled by reflecting surfaces in applications where the receiver is fixed in space. 


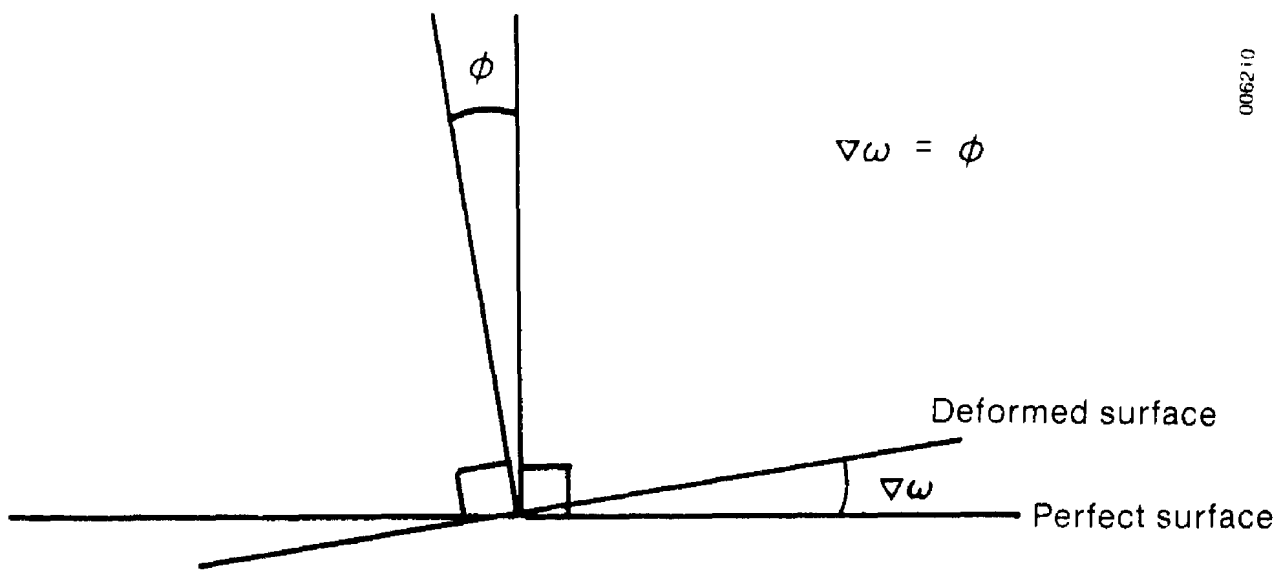

Figure A-1. Definition of the Surface Normal Error ( $\phi)$

where, by comparing Eqs. A-1 and A-2,

$b=-a_{0}$ defines the axisymmetric deformation

$c=l b_{1}-a_{1}$ defines the asymmetric deformation

$d=a_{0}+a_{1}+l b_{0}$

$n=\frac{2 \pi}{\mathrm{P}}$ is the number of equally spaced membrane supports.

The gradient of $w(r, \theta)$ is

$$
\nabla w=\left(\frac{2 b}{a^{2}} r+\frac{c n r^{n-1}}{a^{n}} \cos n \theta, \frac{c n r^{n-1}}{a^{n}} \sin n \theta\right) .
$$

since we are interested in the slope in only the direction of steepest descent, we define the norm of the gradient $|\nabla w|$ :

$$
|\nabla w|=\sqrt{\left(\frac{\partial w}{\partial r}\right)^{2}+\left(\frac{\partial w}{r \partial \theta}\right)^{2}}
$$

therefore

$$
|\nabla w|=\sqrt{\frac{4 b^{2} r^{2}}{a^{4}}+\frac{4 b c n r^{n} \cos n \theta}{a^{n+2}}+\frac{c^{2} n^{2} r^{2 n-2}}{a^{2 n}}} .
$$


As shown in Figure A-1 the surface normal error, $\phi$, corresponding to this deformation and slope is

$$
\phi=\arctan |\nabla w|
$$

Assuming the surface deformation to be small everywhere on the surface of the stretched membrane, we have

$$
\phi \approx|\nabla w|=\sqrt{\frac{4 b^{2} r^{2}}{a^{4}}+\frac{4 b c n r^{n} \cos n \theta}{a^{n+2}}+\frac{c^{2} n^{2} r^{2 n-2}}{a^{2 n}}} .
$$

Case 1: $\quad b=0$ (no axisymmetric deformation)

We now seek to find the deterministic distribution of $\phi$ over the stretched membrane surface. We begin by considering the simple case in which $b=0$. In this case,

$$
\phi=\left|\frac{c n r^{n-1}}{a^{n}}\right| \text {. }
$$

Let $F(\phi \leq z)$ be the fraction of the area of the stretched membrane for which $\phi$ is less than $z$. Without loss of generality we assume $c>0$, so that

$$
\begin{aligned}
& F(\phi \leq z)=F\left(\frac{c n r^{n-1}}{a^{n}} \leq z\right)=F\left[r \leq\left(\frac{z a^{n}}{c n}\right)^{\frac{1}{n-1}}\right] \\
& =F(r \leq h(z)) \text {, } \\
& h(z)=\left(\frac{z a^{n}}{c n}\right)^{\frac{1}{n-1}} ;
\end{aligned}
$$

where

thus

$$
F(\phi \leq z)=\frac{\int_{0}^{2 \pi} \int_{0}^{h(z)} r \partial r \partial \theta}{\int_{0}^{2 \pi} \int_{0}^{a} r \partial r \partial \theta}=\frac{\pi h(z)^{2}}{\pi a^{2}}=\frac{h(z)^{2}}{a^{2}}=\left(\frac{z a}{c n}\right)^{\frac{2}{n-1}} .
$$

The analog of a probability density function, $f_{\phi}(z)$, can be found by differentiating $F(\phi \leq z)$ with respect to $z$ :

$$
f_{\phi}(z)=\frac{\partial F(z)}{\partial z}=\frac{2}{n-1}\left(\frac{a}{c n}\right)^{2 / n-1} z \frac{3-n}{n-1}
$$


The root-mean-square* of $\phi$, RMS ${ }_{\phi}$, of the beam width over the stretched membrane surface is

$$
\text { RMS }_{\phi}=\left[\frac{\int_{0}^{2 \pi} \int_{0}^{a}\left(\frac{c n r^{n-1}}{a^{n}}\right)^{2} r d r d \theta}{\pi a^{2}}\right]^{1 / 2}=\left(\int_{0}^{2 \pi} \frac{c^{2} n}{2 \pi a^{2}} d \theta\right)^{1 / 2}=\frac{c}{a} \sqrt{n} \cdot(A-8)
$$

Having defined the deterministic distribution for this case we now investigate the validity of a probability distribution approximation. We approximate the determiniatic beam width distribution due to surface deformations with a random variable I having a circular normal probability distribution with the same RMS as $\phi$. To this end we define the cumulative distribution $F_{\Gamma}$ :

$$
\begin{aligned}
F_{\Gamma}(z) & =\frac{1}{\sigma_{d}^{2} 2 \pi} \int_{0}^{2 \pi} \int_{0}^{z} \exp \left(-1 / 2 \Gamma 2 / \sigma_{d}^{2}\right) d \Gamma \Gamma d \theta \\
& =\frac{1}{\sigma_{d}^{2}} \int_{0}^{z} \Gamma \exp \left(-1 / 2 \Gamma^{2} / \sigma_{d}^{2}\right) d \Gamma \\
& =1-\exp \left(\frac{-z^{2}}{2 \sigma_{d}^{2}}\right) .
\end{aligned}
$$

To use this approximation we need to express $\sigma_{d}$ in terms of the RMS of $\phi$.

$$
\begin{aligned}
\mathrm{RMS}_{\phi}^{2} & =\mathrm{RMS}_{\Gamma}^{2}=E\left(\Gamma^{2}\right)=\frac{1}{\sigma_{d}^{2} 2 \pi} \int_{0}^{2 \pi} \int_{0}^{\infty} \Gamma^{2} \exp \left(-\Gamma^{2} / 2 \sigma_{d}^{2}\right) d \Gamma \Gamma \mathrm{d} \theta=2 \sigma_{d}^{2} \\
& \text { or } \sigma_{d}^{*}=\operatorname{RMS} \phi / / 2 .
\end{aligned}
$$

As a measure of the error introduced by this approximation, we calculate two error terms, EMAX and ERMS, where

$$
\begin{aligned}
E M A X & =\max _{z}\left|F(\phi \leq z)-F_{\Gamma}(z)\right| \\
& =\max _{z} \mid\left(\frac{z a}{c n}\right)^{\frac{2}{n-1}}+e^{\frac{-z^{2}}{2 \sigma_{d}^{2}}-1 \mid}
\end{aligned}
$$

*RMS is analogous to the second moment of a probability distribution. 
and

$$
\begin{aligned}
\text { ERMS } & =\left\{\int_{0}^{\max \phi} \frac{\left[F(\phi \leq z)-F_{\Gamma}(z)\right]^{2}}{\max \phi} d z\right\}^{1 / 2} \\
& \left\{\int_{0}^{\frac{c n}{a}}\left[\left(\frac{z a}{c n}\right)^{\frac{2}{n-1}}-\left(1-e^{\left.-\frac{z^{2}}{2 \sigma_{d}^{2}}\right)}\right]^{2} d z\right\}^{1 / 2}\right. \\
& =\frac{c n / a}{} .
\end{aligned}
$$

Substituting $x=\frac{2}{\sigma_{d}}$ into Eqs. $A-11$ and $A-12$ gives

$$
\text { EMAX }=\max _{x}\left|\left(\frac{x}{\sqrt{n}}\right)^{\frac{2}{n-1}}+e^{-\frac{x^{2}}{2}}-1\right|
$$

and

$$
\text { ERMS }=\left\{\int_{0}^{\sqrt{2 n}} \frac{\left[\left(\frac{x}{\sqrt{2 n}}\right)^{2 / n-1}+e^{\left.\frac{x^{2}}{2}-1\right] d x}\right.}{\sqrt{2 n}}\right\}^{1 / 2}
$$

Thus when $b=0$ the error introduced by the normal approximation is independent of the values of $a$ and $c$.

Figure A-2 graphically presents the density functions and the cumulative distribution functions for both the circular normal approximation and the deterministic distributions for $n=3$. Figure $A-2$ shows that although the density function of the approximation may be very dissimilar in shape to that of the deterministic distribution when $n=3$, the cumulative distribution functions are nonetheless very close. Figure A-3 compares the deterministic distribution functions for $n=2,3$, and 4. From Figures $A-2$ and $A-3$ it is apparent that the circular normal probability distribution best approximates the $n=3$ case. This is corroborated by the results in Table A-1.

Case 2: $\quad b>0, c=0$ (no asymmetric deformation)

As in the preceding case we begin by defining the deterministic distribution. For this case by Eq. A-3,

*If we express $\Gamma$ in terms of Cartesian coordinates we have $\operatorname{RMS}_{\Gamma}^{2}=E\left(\Gamma^{2}\right)=$ $E\left(\Gamma_{x}{ }^{2}+\Gamma_{y}{ }^{2}\right)=E\left(\Gamma_{x}{ }^{2}\right)+E\left(\Gamma_{y}{ }^{2}\right)=\sigma_{x}{ }^{2}+\sigma_{y}{ }^{2}$, but since $\Gamma$ has a circular normal distribution $\sigma_{x}=\sigma_{y}$ and $\mathrm{RMS}_{\Gamma}{ }^{2}=2 \sigma_{d}{ }^{2}$. Therefore $\sigma_{d}=\sigma_{x}=\sigma_{y}$. 


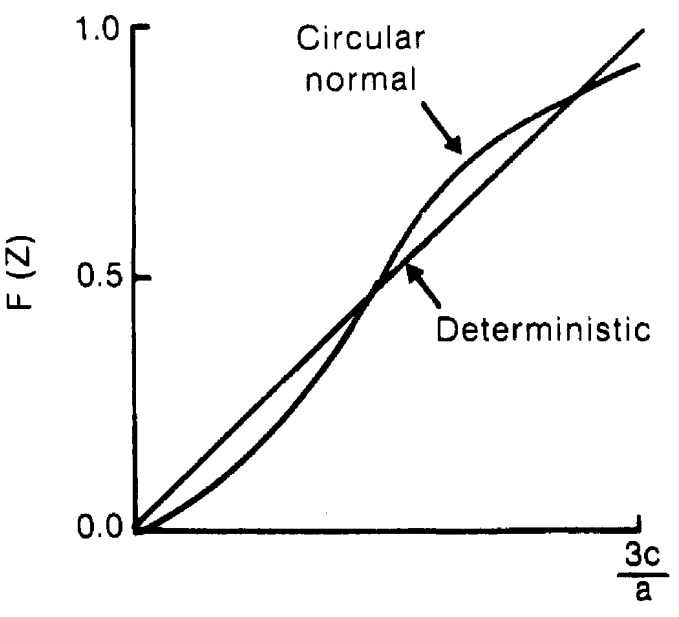

Z

(a) Cumulative Distribution Functions

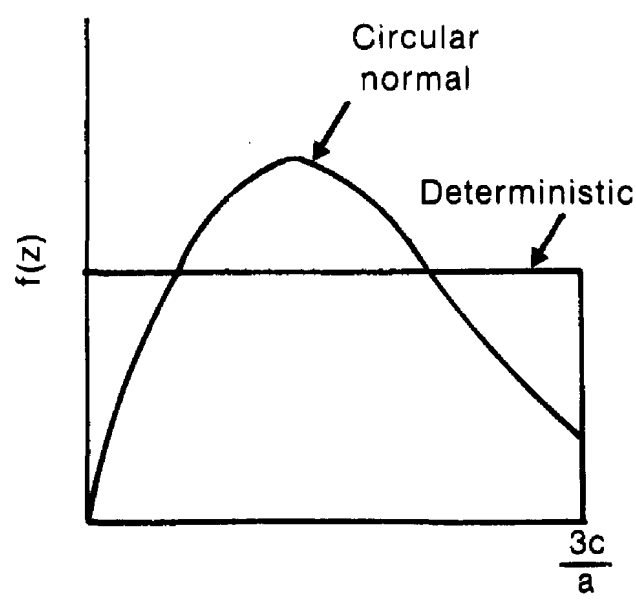

Z

(b) Density Functions

Figure A-2. Comparison of the Deterministic Surface Normal Error ( $\phi)$ Distribution and a Circular Normally Distributed Approximation for $n=3$ and $b=0$

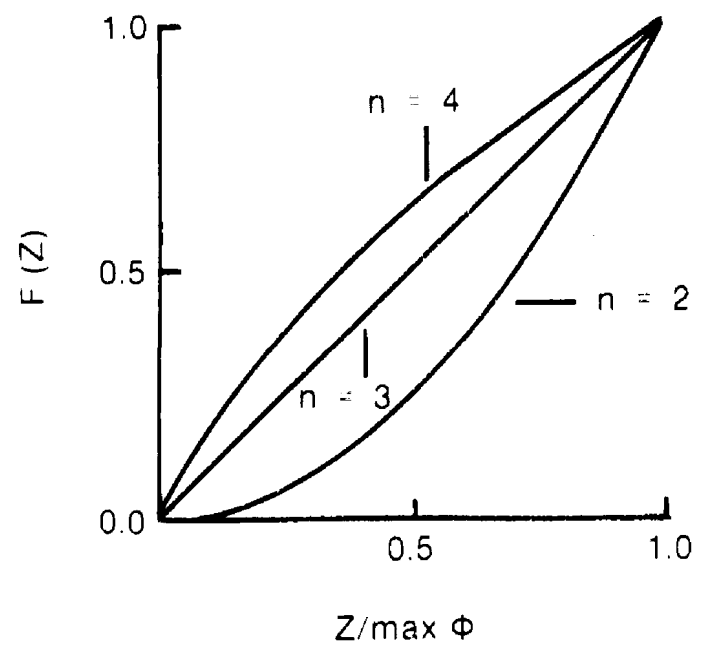

(a) Cumulative Distribution

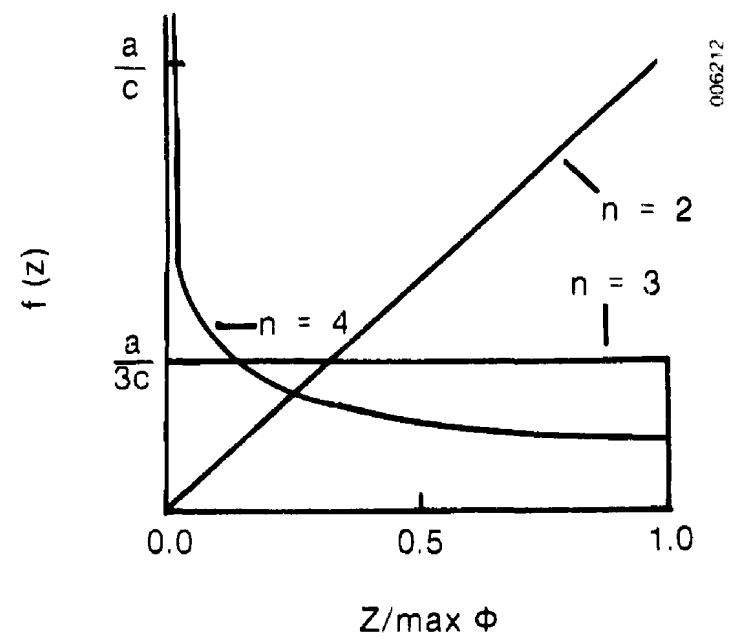

(b) Density Function

Figure A-3. Comparison of the Density and Distribution Functions for $\mathrm{n}=2,3$, and 4 , when $b=0$ 
Table A-1. Errors Introduced by the Assumption of a Normally Distributed Surface Normal

Error when $b=0$

\begin{tabular}{ccc}
\hline $\mathfrak{n}$ & ERMS & EMAX \\
\hline 2 & 0.098 & 0.153 \\
3 & 0.055 & 0.087 \\
4 & 0.104 & 0.198 \\
\hline
\end{tabular}

$$
\phi=\sqrt{\frac{4 b^{2} r^{2}}{a^{4}}}=\frac{2 b r}{a^{2}} .
$$

Given our earlier definition of $F(\phi \leq z)$, we have

$$
\begin{aligned}
F(\phi \leq z) & =F\left(\frac{2 b r}{a^{2}} \leq z\right)=F\left(r \leq \frac{z a^{2}}{2 b}\right) \\
& =F r \leq h(z),
\end{aligned}
$$

where

$$
h(z)=\frac{z a^{2}}{2 b}
$$

Thus

$$
F(\phi \leq z)=\frac{\int_{0}^{2 \pi} \int_{0}^{h(z)} r d r d \theta}{\pi a^{2}}=\frac{z^{2} a^{2}}{4 b^{2}}
$$

The analog of the probability density function, $f_{\phi}(z)$, is again found by differentiating $F(\phi \leq z)$ with respect to $z$ :

$$
f_{\phi}(z)=\frac{z a^{2}}{2 b^{2}}
$$

The root-mean-square, RMS, of the surface normal error over the stretched membrane surface is

$$
\text { RMS }=\left[\frac{\int_{0}^{2 \pi} \int_{0}^{a}\left(\frac{2 b r}{a^{2}}\right)^{2} r d r d \theta}{\pi a^{2}}\right]^{1 / 2}=\left(\frac{\int_{0}^{2 \pi} b^{2} d \theta}{\pi a^{2}}\right)^{1 / 2}=\frac{\sqrt{2} b}{a} \cdot \quad(A-17)
$$


The distribution of $\phi$ in this case has the same form as the case considered earlier in which $b=0$ and $n=2$ (compare Eqs. $A-6$ and $A-15$ as well as Figures $A-3$ and $A-4$ for $n=2$ ). Therefore the errors ERMS and EMAX introduced by the circular normal probability distribution approximation are independent of both $a$ and $b$ and are equal to 0.097 and 0.153 , respectively. These large errors are not surprising given the difference in the density functions shown in Figure $\mathrm{A}-4 \mathrm{~b}$.

Case 3: $b>0, c>0$ (both axisymmetric and asymmetric deformation exist)

As in the previous two cases we begin by defining the deterministic distribution. Let $F_{r}(\phi \leq z)$ denote the fraction of the time that $\phi$ is less than or equal to $z$ on a circle of radius $r$ centered at the center of the circular heliostat. By solving Eq. A-3 for $\theta$ in terms of $\phi$, we can express $F_{r}(\phi \leq z)$ in terms of $\theta$.

$$
\cos n \theta=\frac{\phi^{2}-\frac{4 b^{2} r^{2}}{a^{4}}-\frac{c^{2} n^{2} r^{2 n-2}}{a^{2 n}}}{4 b c n r^{n} / a^{n+2}}=h(\phi, a, b, c, r, n) .
$$

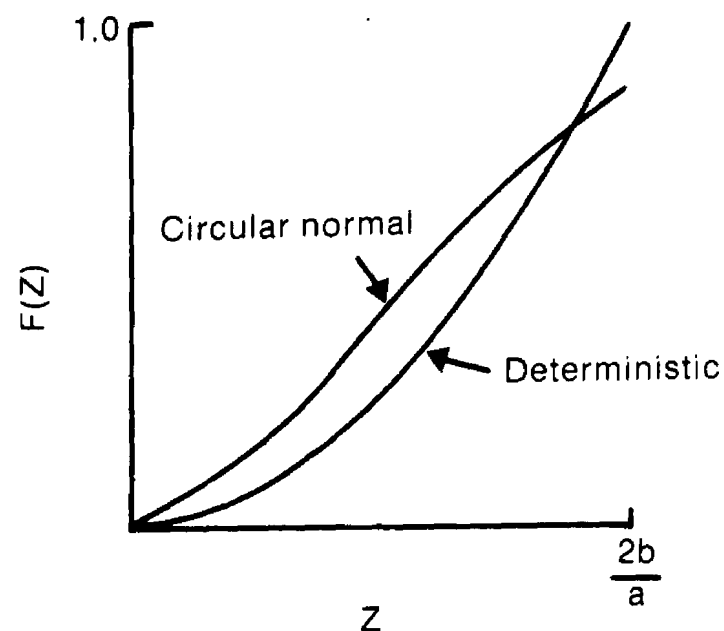

(a) Cumulative distribution

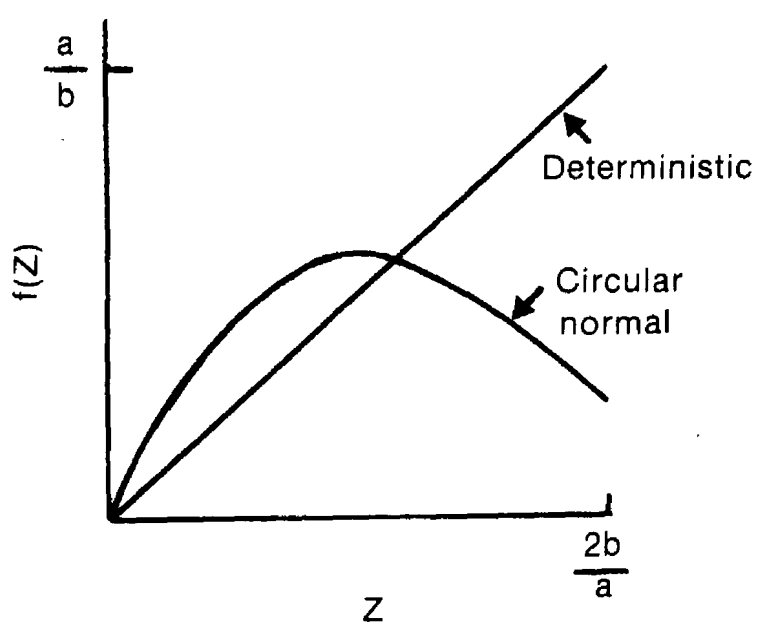

(b) Density function

Figure A-4. Comparison of the Deterministic Surface Normal Error ( $\phi)$ Distribution and a Circular Normally Distributed Approximation for $c=0$ 
Thus if $\phi<z$, then

or

$$
\cos n \theta<h(z, a, b, c, r, n)
$$

$$
F_{r}(\phi \leq z)=F_{r}[\cos n \theta \leq h(z, a, b, c, r, n)] \text {. }
$$

However since $\cos n \theta$ is periodic with period $2 \pi / n$ and symmetric about 0 , we need only consider $\theta$ between 0 and $\pi / n$.

$$
F_{r}(\phi \leq z)=F_{r}[\cos n \theta \leq h(z, a, b, c, r, n) \mid 0 \leq \theta \leq \pi / n] .
$$

Since bc $>0 *$, we have

$$
F_{r}(\phi \leq z)=F_{r}\left[\theta \geq g_{r}(z) \mid 0 \leq \theta \leq \pi / n\right],
$$

where

$$
g_{r}(z)=\frac{1}{n} \arccos [h(z, a, b, c, r, n)] \text {. }
$$

Now employing our earlier definition of $F(\phi \leq z)$ as the fraction of the area of the stretched membrane for which $\phi$ is less than or equal to $z$, we have

$$
\begin{aligned}
F(\phi \leq z) & =\frac{\int_{0}^{a} \int_{r}^{\pi / n}(z) d \theta r d r}{\int_{0}^{a} \int_{0}^{\pi / n} d \theta r d r}=\frac{\int_{0}^{a}\left[\pi / n-\int_{0}^{8} r^{(z)} d \theta\right] r d r}{\frac{\pi a^{2}}{2 n}} \\
& =1-\frac{2}{\pi a^{2}} \int_{0}^{a} r \cdot a r c c o s\left(\frac{z^{2}-\frac{4 b^{2} r^{2}}{a^{4}}-\frac{c^{2} n^{2} r^{2} n-2}{a^{2 n}}}{4 b c n r^{n} a^{n+2}}\right) d r .
\end{aligned}
$$

*For bc $<0, F_{r}(\phi \leq z)$ can still be calculated

$$
\begin{aligned}
& F_{r}(\phi \leq z)=\left[F_{r} \theta \leq g_{r}(z) \mid 0 \leq \theta \leq \pi / n\right] \\
& \text { and } F(\phi \leq z)=\frac{2}{\pi a^{2}} \int_{0}^{a} r \cdot \arccos \left(\frac{z^{2}-\frac{4 b^{2} r^{2}}{a^{4}}-\frac{c^{2} n^{2} r^{2 n-2}}{a^{2 n}}}{4 b c n r^{n} / a^{n+2}}\right) \partial r
\end{aligned}
$$


The root-mean-square, RMS, of the surface normal error over the stretched membrane surface is

$$
\begin{aligned}
\text { RMS } & =\int_{0}^{a} \int_{0}^{2 \pi} \phi^{2} d \theta r d r / \pi a^{2} \\
& =\int_{0}^{2 \pi} \int_{0}^{a}\left(\frac{4 b^{2} r^{3}}{a^{4}}+\frac{4 b c n r^{n+1} \cos n \theta}{a^{n+2}}+\frac{c^{2} n^{2} r^{2 n-1}}{a^{2 n}}\right) d r d \theta / \pi a^{2} \\
& =\left[\int_{0}^{2 \pi} \frac{b^{2} a^{4}}{\left.a^{4}+\frac{4 b c n \cos n \theta}{(n+2)}+\frac{c^{2} n^{2}}{2}\right] d \theta / \pi a^{2}}\right. \\
& =\frac{2 b^{2}}{a^{2}}+\frac{c^{2} n}{a^{2}}
\end{aligned}
$$

or

$$
R M S=\sqrt{\frac{2 b^{2}+c^{2} n}{a}} .
$$

As before we contrast this deterministic surface normal error distribution with that of a surface normal error $\Gamma$ having a circular normal distribution with the same RMS. Table A-2 presents the results for a range of values of $\mathrm{n}$, $a, b$, and $c$ expressed in terms of the errors as calculated by Eqs. A-11 and $A-12$. It can be shown that these errors do not depend on the values of both $b$ and $c$ but only on their ratio. This is evident in the results of Table A-2. These numerically derived results also suggest that, as in the case when $b=0$, the errors are independent of $a$, but we have not proven this.

Convolution of the Surface Deformation Scattering with Other Scattering Effects

The errors shown in Tables $A-1$ and $A-2$ indicate that for a limited number of cases the deterministic distribution of the surface normal error caused by surface deformation is not closely approximated by a normal probability distribution. However, as noted in the introduction to this appendix, the surface deformation is but one of four scattering effects that give rise to the reflected beam width. We briefly investigate the distribution of the sum of the four scattering effects and the error introduced by assuming that sum to be normally distributed.

For this analysis we assume that the other three scattering effects have circular normal distributions, and, therefore, their sum has a circular normal 
Table A-2. Root-Mean-Square Error Results

\begin{tabular}{ccccccc}
\hline $\mathrm{n}$ & $\mathrm{a}$ & $\mathrm{b}$ & $\mathrm{c}$ & $\sigma_{\mathrm{d}}$ & ERMS & EMAX \\
\hline 2 & 5 & 0.001 & 0.001 & 0.00028 & 0.055 & 0.105 \\
2 & 5 & 0.0001 & 0.001 & 0.0002 & 0.093 & 0.145 \\
3 & 5 & 0.001 & 0.001 & 0.00032 & 0.070 & 0.143 \\
3 & 5 & 0.0001 & 0.001 & 0.00025 & 0.053 & 0.086 \\
3 & 5 & 0.001 & 0.0001 & 0.0002 & 0.087 & 0.143 \\
3 & 5 & 0.0003 & 0.003 & 0.00074 & 0.053 & 0.086 \\
3 & 5 & 0.00003 & 0.003 & 0.00073 & 0.056 & 0.087 \\
3 & 10 & 0.001 & 0.001 & 0.00016 & 0.070 & 0.143 \\
3 & 10 & 0.001 & 0.0001 & 0.0001 & 0.087 & 0.143 \\
\hline
\end{tabular}

distribution. Thus the distribution of this sum in a single dimension has a normal distribution with mean 0 and standard deviation $\sigma$. We convolve this normal distribution in one dimension* with the distribution of scattering caused by surface deformation. Finally, we investigate the error introduced by assuming a normal distribution as an approximation for this convoluted sum.

We examine the case $b=0, n=2$ for which the errors introduced by a circular normal approximation were greatest (see Tables A-1 and A-2). For this case the deterministic cumulative distribution function $F_{1}$ for the surface normal beam width in a single dimension (see $\mathrm{Eq} . \mathrm{A}-5$ ) is

$$
F_{1}(\phi \leq z)=F_{1}\left(r \leq \frac{z a^{2}}{2 c}\right)=\frac{\int_{-a}^{\frac{z a^{2}}{2 c}} d r}{\int_{-a}^{a} d r}=\frac{z a}{4 c}+0.5
$$

*The convolution and errors are computed in only a single dimension due to the numerical complexities of the two-dimensional convolution of a circular normal distribution with the deterministic distribution. A small error in one dimension implied a small error in the two-dimensional approximation. 
and the corresponding density function is

$$
f_{1}(z)=\frac{a}{4 c}
$$

Since the scattering error introduced by surface deformation of a reflecting surface is approximately twice the size of the surface normal error, the maximum scattering effect caused by surface deformation is $2 \times \frac{2 c}{a}=\frac{4 c}{a}$ and the corresponding density function is

$$
f_{1}(z)=\frac{1}{2} \times \frac{a}{4 c}=\frac{a}{8 c} .
$$

Convoluting this density with that of a normal distribution with mean 0 and standard deviation $\sigma$ yields the single dimension density function, $h(y)$, of the sum:

$$
h(y)=\int_{\frac{-4 c}{2}}^{\frac{4 c}{2}} \frac{1}{\sigma \sqrt{2 \pi}} e^{-\frac{(y-z)^{2}}{2 \sigma^{2}}} \frac{a}{8 c} d z
$$

The probability that the scattering beam width is less than $x$ is found from the cumulative distribution function:

$$
H(x)=\int_{-x}^{x} h(y) d y=\frac{a \sigma}{4 c} \int_{\substack{x \\ \sigma}}^{\frac{x}{\sigma}+\frac{4 c}{a \sigma}} N(v) d v-1,
$$

where $N(v)$ is the cumulative normal distribution function with mean 0 and sţandard deviation of one. The standard deviation of this convoluted sum, oref1, is

$$
\sigma_{\text {ref } 1}^{\prime}=\left(\frac{16 c^{2}}{3 a^{2}}+\sigma^{2}\right)^{1 / 2}
$$

We can now compare the distribution of this convoluted sum with a normally distributed approximation with mean 0 and standard deviation $\sigma_{\text {ref } 1}$. Table A-3 shows the errors introduced by the normal approximation for several values of $a, c$, and $\sigma$. Although the error term, EMAX, was calculated using Eq. A-11, it should be noted that this error is not strictly comparable to those calculated earlier because it is measured in only a single dimension. Nonetheless, as shown by the results in Table A-3, convolution with a normal distribution with $\sigma \geq c$ significantly dampens any errors introduced by a normal approximation for the scattering effects caused by surface deformation. 
Table A-3. Error Introduced in a single Dimension by a Normal Approximation when $b=0, n=2$

\begin{tabular}{rccc}
\hline$a$ & $c$ & $\sigma$ & EMAX \\
\hline 5 & 0.001 & 0.001 & $<0.01$ \\
5 & 0.001 & 0.0005 & 0.015 \\
5 & 0.001 & 0.0001 & 0.103 \\
10 & 0.001 & 0.001 & $<0.01$ \\
10 & 0.001 & 0.0001 & 0.076 \\
5 & 0.002 & 0.001 & 0.015 \\
\hline
\end{tabular}

\section{Conclusions}

A circular normal approximation for the deterministic distribution of surface deformation scattering effects can be in error by a significant amount for some cases. However, when the surface deformation scattering effects are added to the scattering effects caused by the mirror material, tracking errors, and concentrator size cone, the error introduced by the normal approximation is considerably dampened. 


\section{APPENDIX B \\ ANTICIPATED LOADING AND THE RESULTING SURFACE ERRORS POR INDIVIDUAL STRETCHED MEMBRANE MODULES}

The following degcription provides a rationale for the assumed approximate levels of wind and weight loading on stretched membrane reflector modules as well as for the resulting surface slope errors. We first address the expected loading levels on stretched membrane modules, and follow this with a discussion of the surface normal errors resulting from that assumed loading for typical but not optimal designs. Only a cursory presentation is included here as the analysis results for the estimated surface errors for structurally deformed stretched membrane modules are currently being documented in detail by Murphy [5] and Murphy et al. [6].

To arrive at an estimate of the wind effects on the membrane heliostat reflectors we consider only steady-state wind loads. Further, we assume that the total loading normal to the module surface from the combined effects of wind, vacuum focusing, and weight is uniform over the surface, though some spacial and temporal nonuniformity in the wind-induced load is sure to exist. The degree of nonuniformity in the steady-state wind pressure distribution and the dynamic turbulent effects, for either single or fields of heliostats (including glass/metal concepts), are not known at this time nor have the impacts of the resulting problems from these effects been analyzed thoroughly. However, based on preliminary and simplified analyses, good design approaches can probably mitigate to a great extent the deleterious effects arising from spatially nonuniform and turbulent wind loading. Further, since the wind loading is only a portion of the total load, and often not the dominant portion, the impacts from these effects should be further decreased. This is clearly an area for further evaluation.

We consider two kinds of surface errors: that corresponding to the axisymmetric deformation (RMS ${ }_{A X}$ ) and that corresponding to asymmetric deformation (RMS ${ }_{A S}$ ). RMS ${ }_{A X}$ corresponds to axisymmetric deformation when the membrane edge attachment is held fixed with zero displacement. RMS AS occurs as a result of the displacement of the membrane edge; this edge displacement in turn results from the out-of-plane deformation of the frame on which the membrane is stretched. Further, because a vacuum control of the plenum chamber between the membranes is assumed to be operative and effective in eliminating RMS for focused designs, only RMS will be of concern for these focused designs. Both RMS $A X$ and RMS ${ }_{A S}$ will be of concern for the unfocused design.

Whether considering axisymmetric (RMS ${ }_{A x}$ ) or asymmetric (RMS ${ }_{A S}$ ) surface error contributions, the error is proportional to the effective load per unit area (defined somewhat differently for each case) on the collector. This is because for either single or double membrane concepts both the frame and the membrane deform nearly linearly with pressure $[5,6]$. For this analysis we consider only double membrane concepts because of the structural efficiency offered by this design approach. 
For the axisymmetric contribution, the expression for the root mean square surface error RMS $A X$ in terms of the load per unit aperture area of collector $\mathrm{P}_{\mathrm{AX}}$, is given, from simple linear membrane deformation analysis, by the approximation*

$$
\text { RMS }_{\mathrm{AX}}=\mathrm{C}_{1} \mathrm{P}_{\mathrm{AX}}
$$

where $C_{1}$ is defined by

$$
c_{1}=\frac{\sqrt{2 a}}{4\left(T_{0} / 2\right)}=\frac{\sqrt{2 a}}{2 T_{0}}
$$

$T_{0}$ is the total tension load on frame applied by the two membranes (each is assumed to have the same tension).

$\mathrm{P}_{\mathrm{AX}}$ is described as

$$
\mathrm{P}_{\mathrm{AX}}=W_{\mathrm{m}} \cos \theta_{\mathrm{z}}+\frac{1}{2}\left(\mathrm{P}_{\mathrm{NW}}\right)
$$

where $\mathrm{P}_{\mathrm{NW}}$, the wind-induced pressure normal to the surface, is approximated by

$$
P_{N W}=C_{N} \frac{1}{2} \rho v^{2} \sin \theta=C_{N} q \sin \theta \approx q \sin \theta \text {. }
$$

$W_{m}$ is the weight/area of the front reflector membrane and a is the radius of the membrane: $C_{N}$ is the loading coefficient which we have assumed to be 1.0 . For a single isolated heliostat the value of $\mathrm{C}_{\mathrm{N}}$ may be as high as 1.5 , but for fields of heliostats recent data indicate that $C_{N}$ may be considerably below 1.0. $q$ is defined as the dynamic wind pressure $\left(=1 / 2 \rho^{2}\right)$. $\theta_{2}$ is the collector zenith angle as shown in Figure $B-1$. The expression for $\mathrm{P}_{A X}$ (and $\mathrm{P}_{A S}$, which is defined below) assumes that the wind angle of attack is the complement of the collector zenith angle $\theta$, but not the yaw angle (rotation about the vertical axis in Figure B-1), which is assumed to be zero.

$\mathrm{P}_{\mathrm{AX}}$ is composed of two contributions, the weight on the reflector membrane, and that caused by the wind velocity induced pressure on the reflector surface. Two points are worthy of note. First, only the reflector membrane is considered in the weight contribution since only the reflector is of concern in the axisymmetric contributions (we do not really care how the rear membrane distorts). Second, the total pressure load on the collector caused by wind is assumed to be made up of two contributions of equal magnitude; i.e., a positive increase on the windward side of the collector and a negative

*This approximation is valid whether the displacement increment is measured from an initially curved shape or from an initially flat condition. For the unfocused heliostats we will be returning to deviations from the flat condition and will actually be penalizing the system for a benefit which results from gravity focusing. 


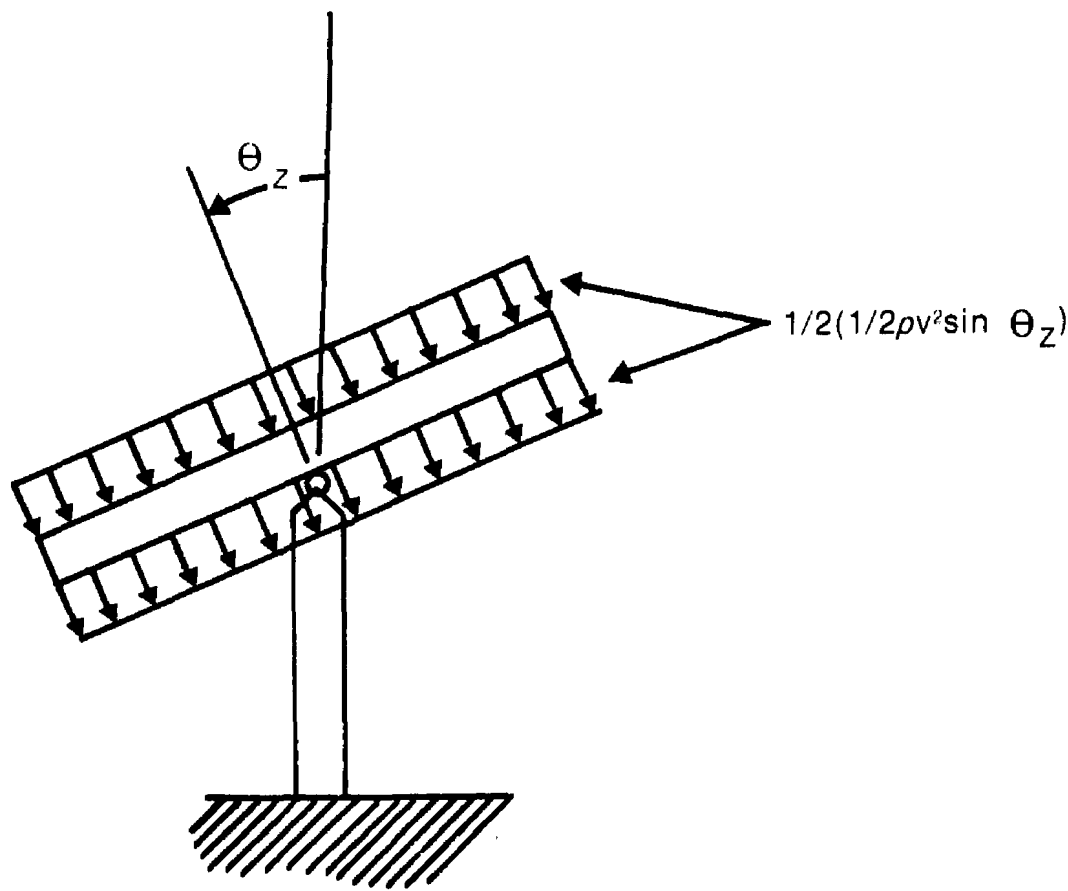

Eigure B-1. Asgumed Uniform Pressure Loading on the Double Membrane Collector

contribution on the leeward side of the collector (see Figure B-1). Thus, with the double membrane design, the effective pressure on the reflector surface is only one half of the dynamic wind pressure. Note also that the effect of the double membrane is considered in the expression for $C_{1}$ where the $T_{1}$ is divided by 2 . The magnitude of the orientation angle of the collector is discussed below.

For the asymmetric deformation, the surface error RMS AS can be expressed as*

$$
B_{2}=C_{2} P_{A S} \text {, }
$$

where $\mathrm{C}_{2}$ is a constant depending on the geometry and specific design of the heliostat and $P_{A S}$ is the load per unit area given. This form considers only the loads normal to the plane of the collector. ${ }_{A}$ AS is given by

$$
P_{A S}=W_{A} \cos \theta_{z}+\frac{1}{2} \rho v \sin \theta_{z}=W_{A} \cos \theta_{z}+q \sin \theta_{z},
$$

where $W_{A}$ is the weight per unit reflector area of the whole module assembly. Note that the pressure load considered here is the full dynamic wind pressure, where both the windward and leeward contributions must be considered. This is because the asymmetric error contribution is caused by the periodic reaction

*Though it is not obvious from the 1 imited discussion given here, numerous previous analyses $[4,5,6]$ have demonstrated the adequacy of using this form. 
forces, which must oppose the entire wind and weight load normal to the plane of the reflector.

A value for the wind-induced pressure must be determined. One measure for that quantity, which is used in this analysis, is the expected value of the wind-induced pressure; $i . e .$, the dynamic pressure weighted average of the wind probability distribution. Such wind probability distributions are available for selected locations $[17,18]$ from previous studies. In these studies, the total amount of insolation corresponding to a specified velocity interval is given, and so the probability density of insolation as function of velocity, $f(v)$, can be determined.* Then the expected value of the wind induced pressure $q$ can be determined from

$$
\bar{q}=\frac{1}{2} \rho \int_{0}^{\infty} v^{2} f(v) d v=\frac{1}{2} \rho \overline{v^{2}}
$$

where it is assumed that the air density $p$ is constant (i.e., not dependent on the velocity $v$ at the site).

Thus, for a given value of collector attitude, the expected values of $P_{A X}$ and ${ }^{P}$ AS can be determined by

$$
\bar{P}_{A X}=\int_{0}^{\infty} P_{A X} f(v) d v=W_{M} \cos \theta_{z}+\frac{1}{2} \bar{q} \sin \theta_{z}
$$

and

$$
\bar{P}_{A S}=\int_{0}^{\infty} P_{A S} f(v) d v=W_{A} \cos \theta_{z}+\bar{q} \sin \theta_{z} .
$$

$q$ is easily evaluated with Eq. B-7 along with the field data from McDonnell Douglas [18]. The results of this evaluation along with some other informative statistics are given in Table B-1 for several locations and a "strawman" composite site, which is synthesized by averaging the insolation density functions for the seven sites considered in the study [18].

It is interesting to note from Table B- 1 that when using $\left(\overline{v^{2}}\right)^{1 / 2}$ as the measure of velocity in the cumulative distributions, nominally $70 \%$ of the insolation at a given location occurs at or below this velocity whereas using the average velocity $\bar{v}$ the respective insolation level is approximately $60 \%$.

RMS $A$ and RMS $A S$ can now be determined for a particular design using the above definition of $q$ along with Eqs. B-7 and B-8, known weight properties of the module, and a given angle of attack.

$\bar{P}_{\text {PX }}$ is shown as a function of $\theta$ for Barstow for several membrane designs in Figure B-2. At low angles of attack ( $\theta$ small), weight is the primary contribution to $\overline{\mathrm{P}}_{\mathrm{AX}}$, while for large values $\left(\theta\right.$ near $\left.90^{\circ}\right), \overline{\mathrm{P}}_{\mathrm{AX}}$ is primarily due to

*Another description for $f(v) d v$ is the fraction of solar energy that occurs for wind velocities in the range of $v$ and $v+d v$. 
Table B-1. Selected Wind Velocity Characteristics for Several Locations ${ }^{a}$

\begin{tabular}{lcccccc}
\hline Location & $\begin{array}{c}\bar{v} \\
(\mathrm{~m} / \mathrm{s})\end{array}$ & $\begin{array}{c}\overline{\mathrm{v}^{2}} \\
(\mathrm{~m} / \mathrm{s})^{2}\end{array}$ & $\begin{array}{c}\overline{\bar{v}}=\left(\sqrt{\mathrm{v}^{2}}\right) \\
(\mathrm{m} / \mathrm{s})\end{array}$ & $\begin{array}{c}\mathrm{q} \\
(\mathrm{Pa})\end{array}$ & $F(\overline{\mathrm{v}})^{\mathrm{b}}$ & $F(\overline{\bar{v}})^{\mathrm{b}}$ \\
\hline Albuquerque & 3.89 & 22.37 & 4.73 & 13.70 & 0.601 & 0.713 \\
Phoenix & 3.47 & 15.13 & 3.90 & 9.26 & 0.555 & 0.634 \\
Barstow & 5.14 & 34.97 & 5.91 & 21.39 & 0.613 & 0.692 \\
$\begin{array}{l}\text { Composite of } \\
\text { seven sites }\end{array}$ & 4.46 & 27.57 & 5.25 & 16.89 & 0.585 & 0.708 \\
\hline
\end{tabular}

a Based on data from MeDonnell Douglas [18].

$b_{F(v)}$ is defined as the cumulative fraction of insolation obtained at $a$ site for all wind velocities up to and including $v$.

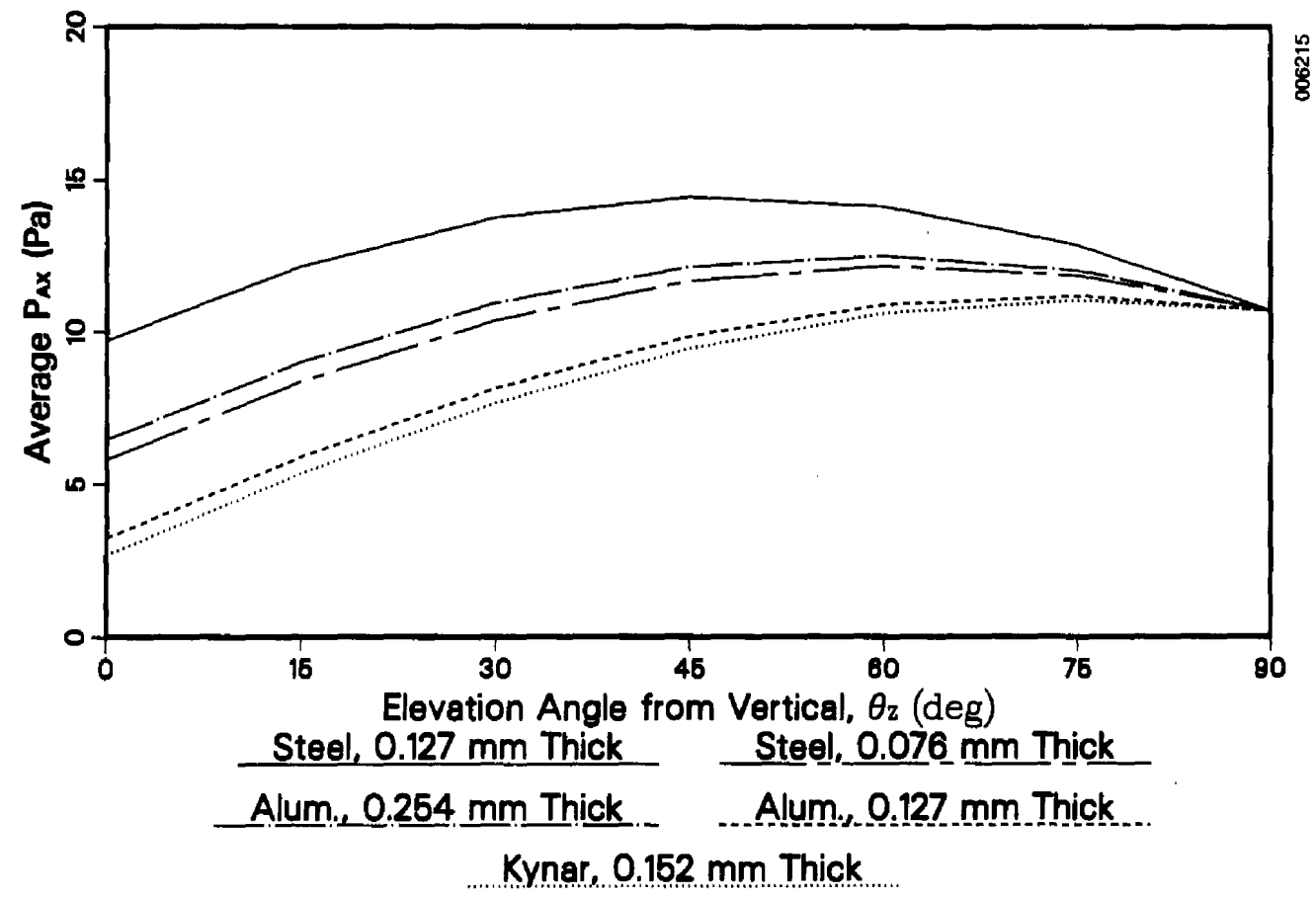

Eigure B-2. Effective Expected Module Load Corresponding to Arisymmetric Membrane Deformation as a Function of Zenith Angle for Several Membrane Designs 
wind pressure loading. It is noted that the wind-induced pressure on the reflector membrane is only one half of the expected value of the dynamic wind pressure at the Barstow site since the module is a two membrane design. For designs with heavy membranes (i.e., thick steel concepts), the weight will be the dominant factor in determining the effective load for a wide range of angles of attack. On the other hand, if the membrane is thin or of a lightweight material, such as aluminum or polymer, then the effective load will be dominated by the wind effect. In fact, a reasonable design pressure 1 imit for "good" unfocused designs would appear to be the effective pressure load corresponding to the wind only.

Eigure B-3 shows the RMS axisymmetric error contribution, RMS $A X$, as a function of $\bar{P}_{\text {AX }}$ for several module designs. Here it is seen that once the effective load is determined, the RMS error depends only on the membrane module radius geometry and tension level. If we use as the measure of effective pressure load, the value corresponding to wind only effects, then a reasonable upper level of error for RMS $A X$, corresponding to "good" design, is about 2 mrad for the Barstow location. From the data in Table B-1 it is seen that for the other locations and for the "composite" site the corresponding level will be somewhat lower. Further, for advanced designs there may be the potential to design for error levels that are considerably lower than the $2 \mathrm{mrad}$.

The situation for the asymmetric load contribution $\overline{\mathrm{P}}_{\mathrm{AS}}$ is considerably different than that for $\overline{\mathrm{P}}_{\mathrm{AX}}$ since the weight of module, though very low when compared to glass/metal concepts, will in general be considerably greater than

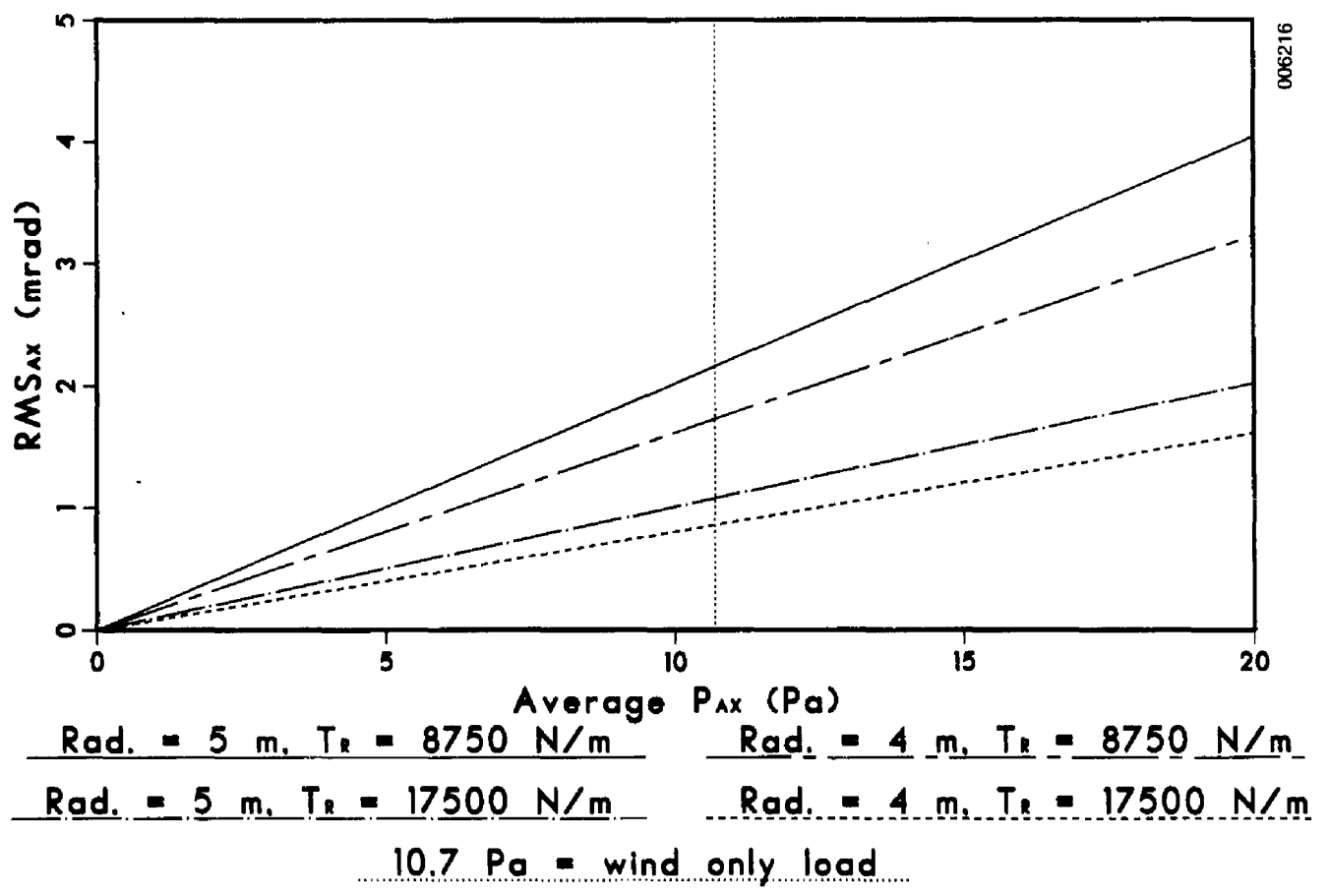

Figure B-3. Effective RMS Surface Error as a Function of $\overline{\mathrm{P}}_{\mathrm{AX}}$ for Several Model Designs 
the reflector membrane only. Typical steel module designs with areal weights of around $100 \mathrm{~Pa}\left(2.09 \mathrm{lb} / \mathrm{ft}^{2}\right)$ have been configured, while reflector membranes well below $20 \mathrm{~Pa}\left(0.42 \mathrm{lb} / \mathrm{ft}^{2}\right)$ appear feasible. Figure B-4 illustrates these points where the expected areal load for the module, corresponding to asymmetric deformation, is shown as a function of collector elevation angle for several assumed module weights $(75,100$, and $125 \mathrm{~Pa}$ corresponding to 1.57 , 2.09 , and $2.61 \mathrm{lb} / \mathrm{ft}^{2}$ ). The lowest curve for $33 \mathrm{~Pa}$ shown in Figure $\mathrm{B}-4$ corresponds to an aluminum design that is similar in construction to the 100-Pa steel designs. The weight is clearly the dominant factor for all of these cases corresponding to the Barstow wind data. Hence, this led us to select a reasonable lower limit of $30^{\circ}$ for the zenith angle for the asymmetric load design criteria (this will tend to result in conservatively high weight loads).

In making the surface error structural analysis computations corresponding to the asymmetric error effects, a zenith angle of $30^{\circ}\left(\cos 30^{\circ}=0.87\right)$ and an applied pressure of $10 \mathrm{~Pa}$ (corresponding to a mean dynamic pressure of $\frac{1}{2} \rho v=10 / \sin ^{2} 30=20 \mathrm{~Pa}$ at $\left.\theta_{z}=30^{\circ}\right)$ were selected. It was felt that this combination represented a reasonable average total load corresponding to asymmetric deformation.

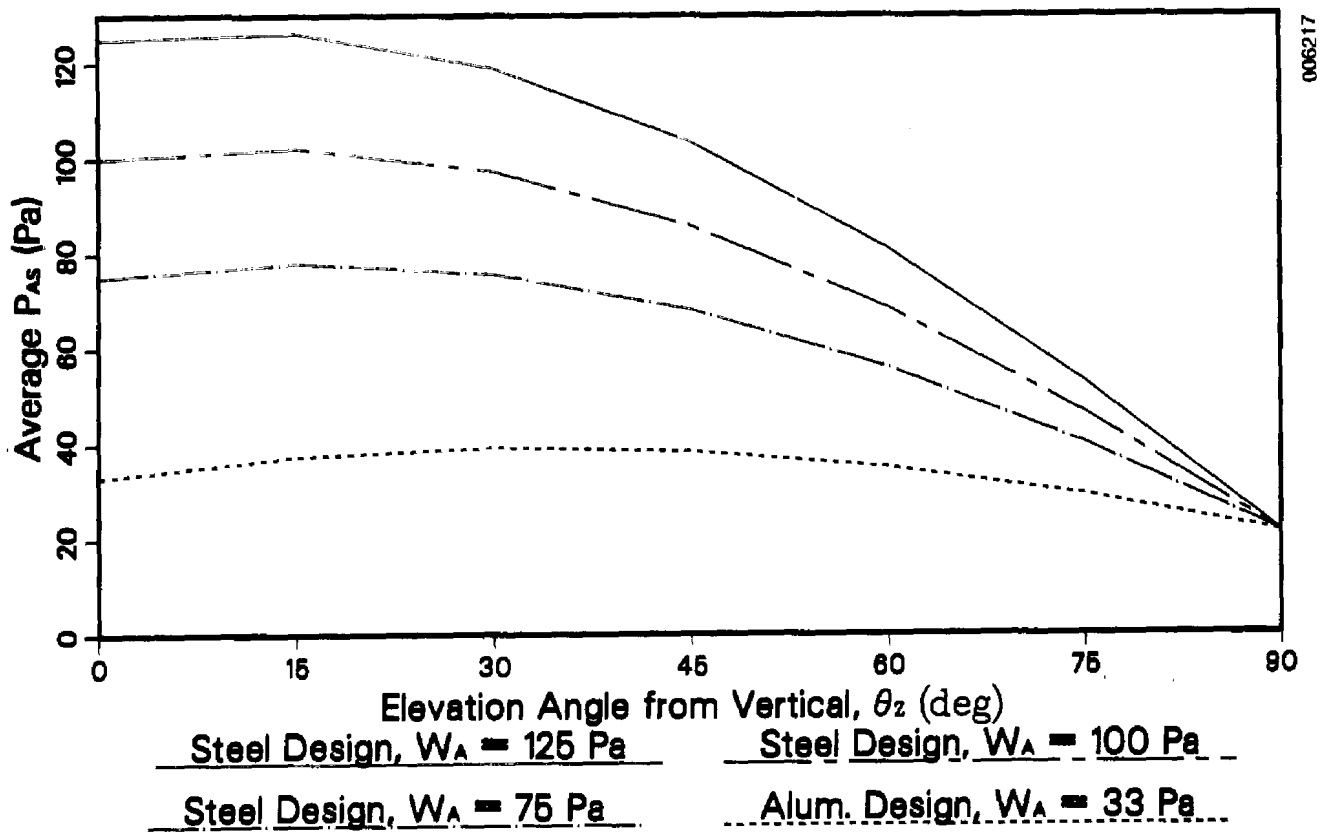

Bigure B-4. Average Load Corresponding to Asymetric Loading for Several Model Designs 
The asymmetric error level associated with these assumed loads (see Figure $\mathrm{B}-5$ ) is described next. The results illustrated in Figures $\mathrm{B}-7$ through B-10 show typical structural analysis results as calculated with the NASTRAN finite element method [19] and with a more simple analytical approach as described in [5]. The phenomen leading to these results are described much more extensively in Murphy et al. [5] and Murphy [6]. Furthermore, the results demonstrate that these load induced error levels can potentially be controlled to quite low levels. The modules considered are shown in Figure B-6; i.e., steel construction, 5-m radius, 0.254-mm-thick membranes, $2 \mathrm{~h} \times 76.0-\mathrm{mm}$ frames with 3.048-mm-thick walls. Figure B-7 shows how the bending and torsional rigidity vary with frame height for the selected frame design.

Figure B-8 illustrates for several module designs how the peak frame deflection (midway between the supports) varies as a function of frame height for the assumed loading on the module. The curve with the highest deformation corresponds to a single membrane design, and the next lower curve corresponds to a double membrane design with attachments that can slide circumferentially but not radially. The lowest overall deflection curve corresponds to a double membrane design with hard attachments (bounds or welds) to the frame. The mechanics of why these modules behave so differently will not be discussed here (see Murphy et al. [6]). However, it is clearly seen that module performance can be dramatically improved with different design approaches, and very

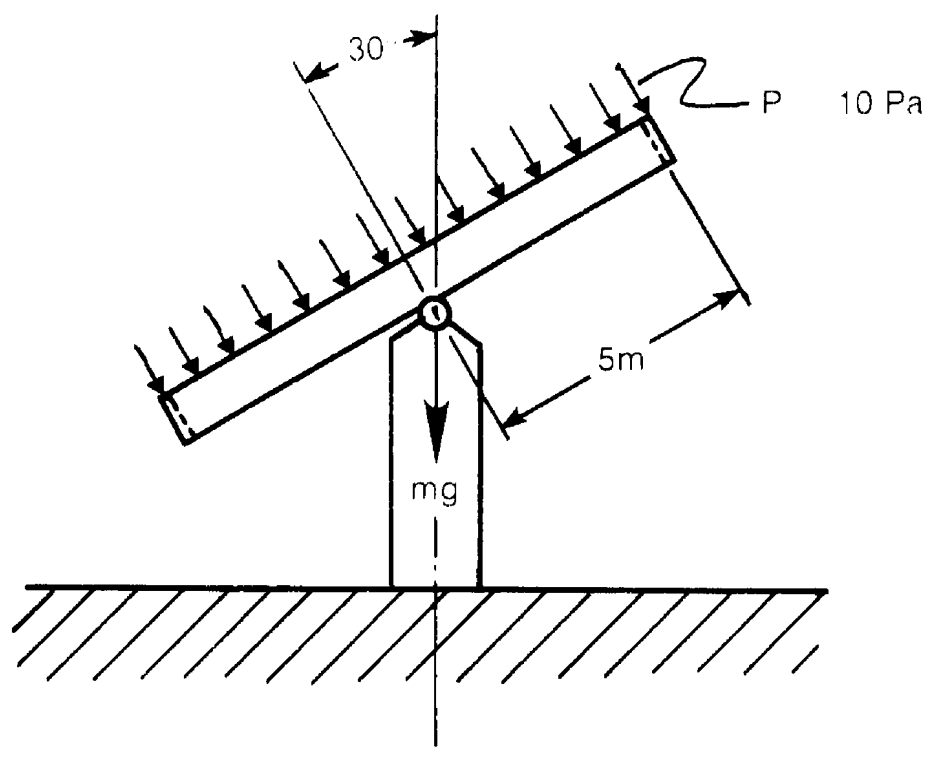

Figure B-5. Effective Expected Module Area Load Corresponding to Asymetric Deformation as a Function of Zenith Angle for Several Module Weights 


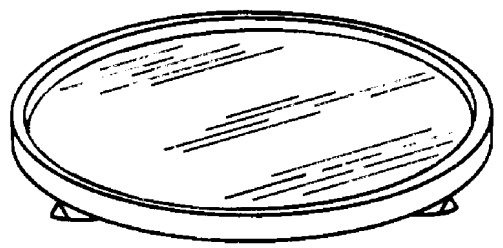

(a) Perspectlve view - stretched-membrane reflective module; pin supported at three equidistant circumferential points

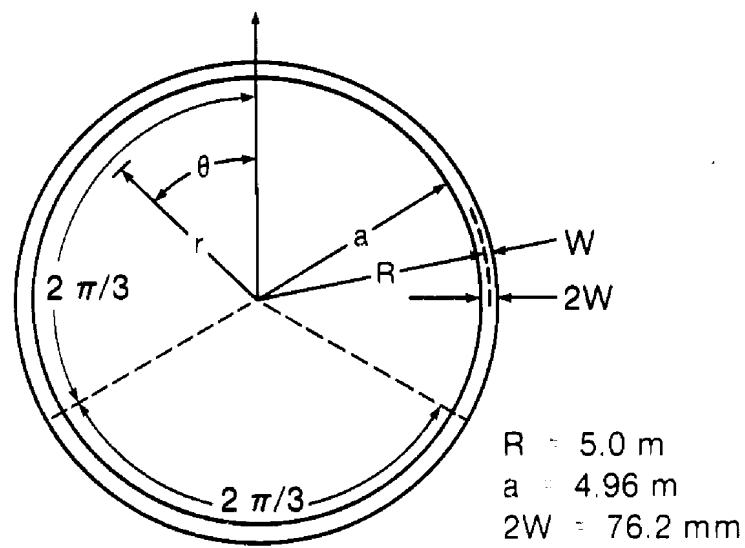

(b) Top view - stretched-membrane reflective module
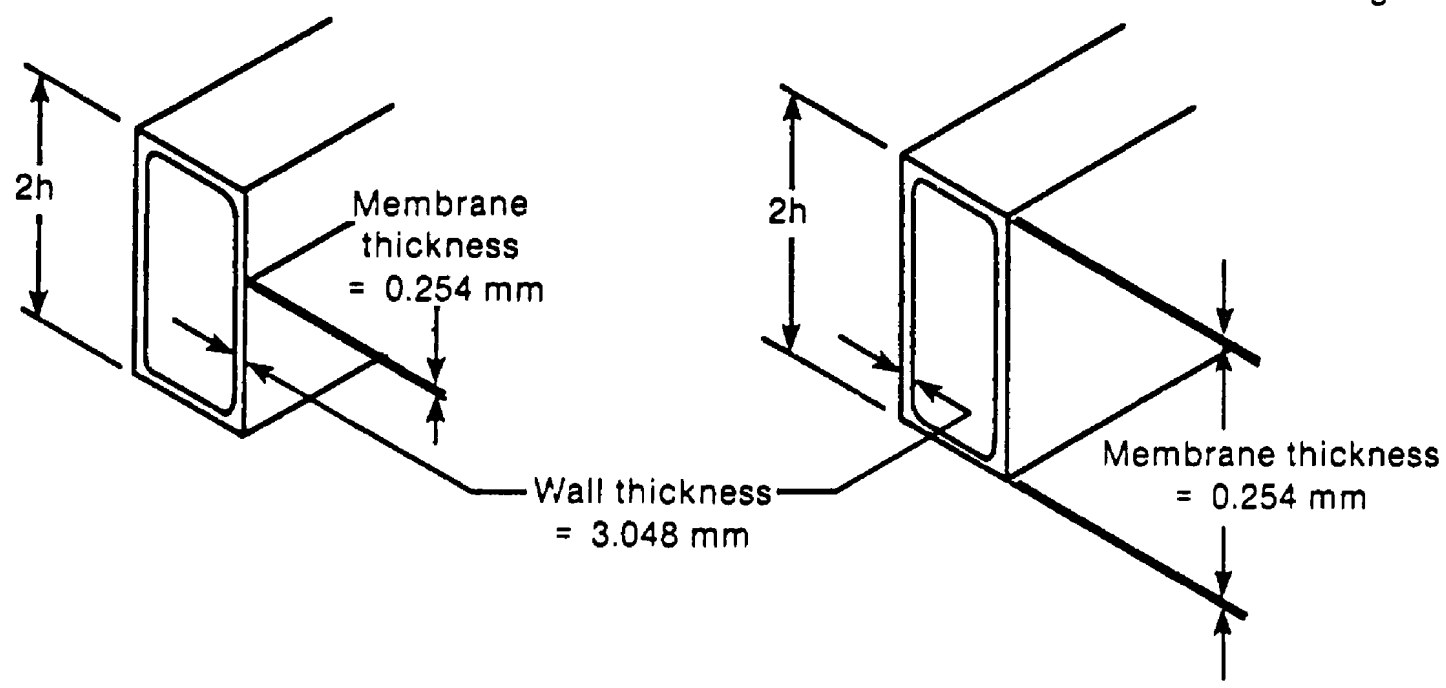

(c) Cross-section view for steel frame/membrane combination considered in analysis discussion

Figure B-6. Example of Module Construction 
small deformation limitations appear possible. The way in which these deformations translate into surface errors is seen in Figures $B-9$ and $B-10$, where the asymmetric error contribution (RMS ${ }_{A S}$ ) for the three designs discussed in Figure B-7 are plotted as a function of frame half-heights (in Figure $B-9$ ) and then as a function of total module areal weight (in Figure $B-10$ ). It is seen that the asymmetric error, RMS AS, can potentially be controlled to better than about $0.5 \mathrm{mrad}$ (rms) at a module areal weight of about $100 \mathrm{~Pa}$ for the steel design shown. It might also be noted that for aluminum designs the same error limitation appears attainable for about $1 / 3$ the weight [6].

Based on these findings the macroscopic surface shape for the focused stretched membrane modules appears to be potentially as good as that corresponding to the state-of-the-art glass/metal heliostats when only the asymmetric error contribution is considered. More specifically our calculations show the potential to limit wind- and weight-induced deformations to about $0.5 \mathrm{mrad}$ rms total for the asymmetric portion, and this appears to be at least as good as typical glass/metal designs. It is assumed that the axisymmetric contribution caused by uniform pressure is negligible. However, the control of the plenum chamber may fall significantly short of this goal when nonuniform pressure loads are considered. This area remains a significant unknown.

On the other hand, the surface errors for the unfocused stretched membrane heliostats can potentially be larger because of the axisymmetric contribution which is not considered to exist in the focused controlled stretched membrane

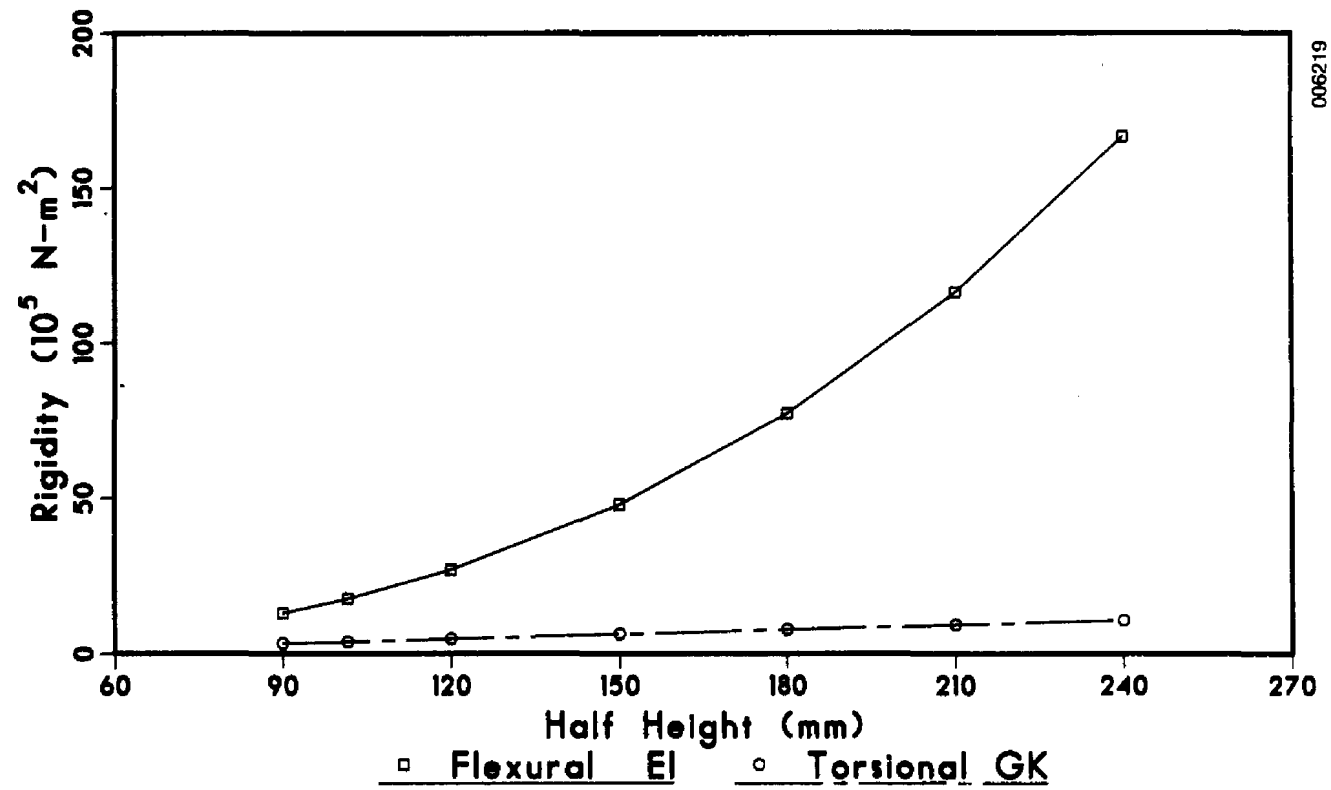

Figure B-7. Torsional and Flexural Rigidity as a Function of Erame Half Height 


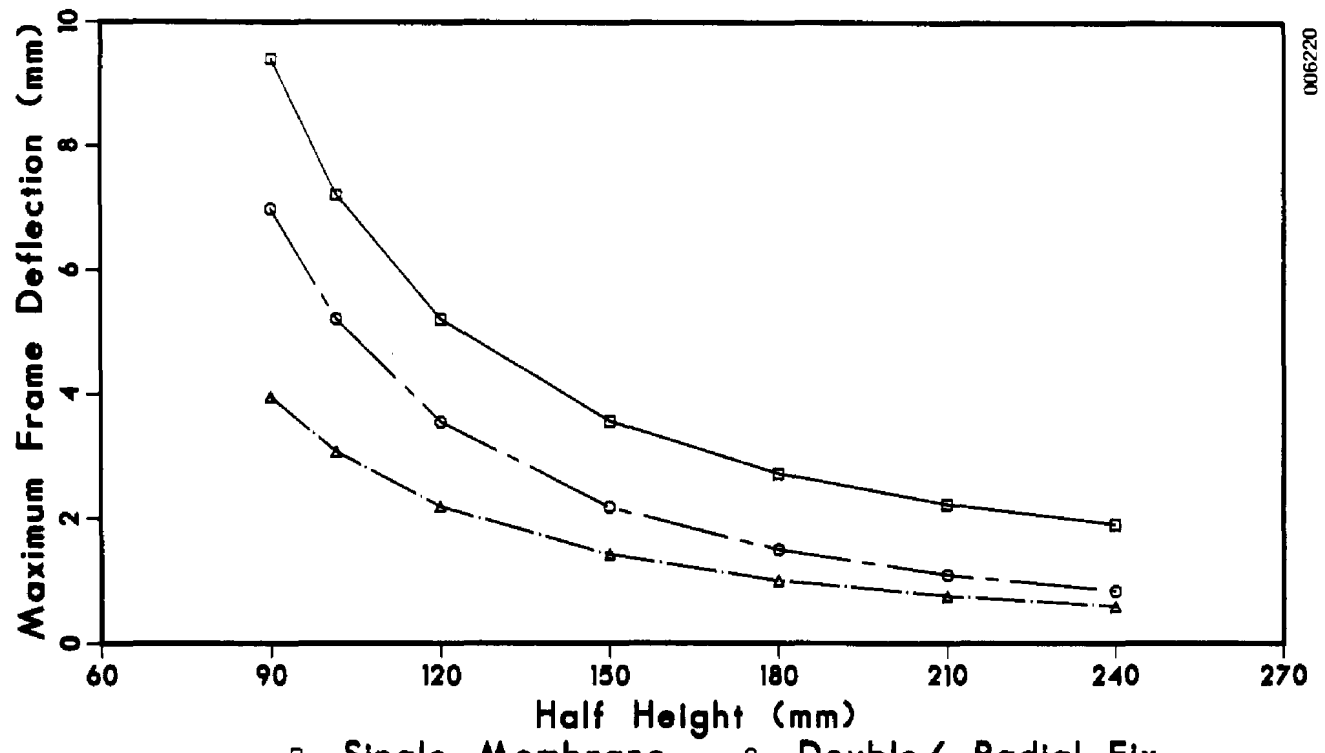

- Single Membrane o Double/ Radial Fix

$\triangle$ Double/ Rodial \& Circum. Fix

Pigure B-8. Maximum Frame Deflection as a Runction of Frame Half Height for Several Construction Types

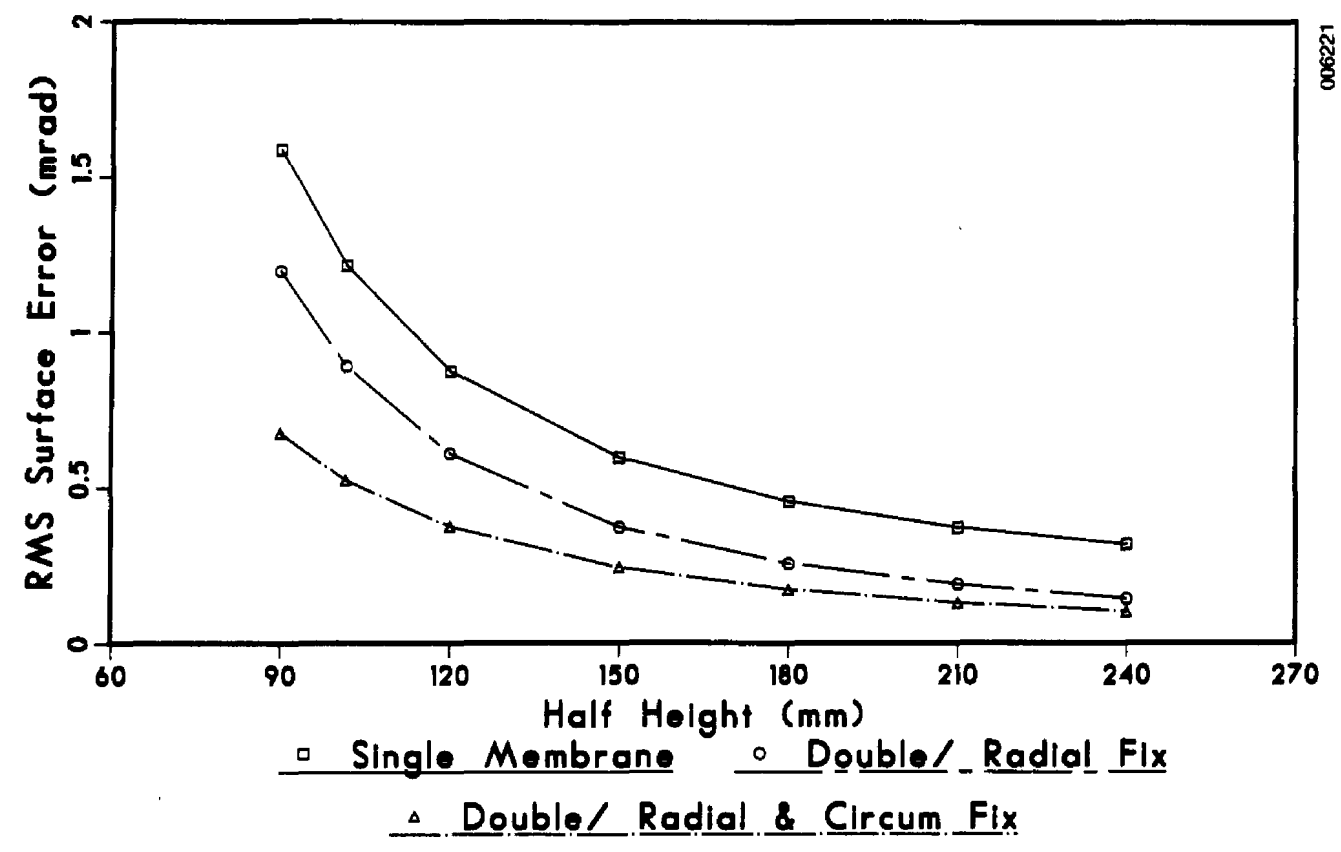

Figure B-9. RMS (Surface Averaged) Error as a Function of Frame Half Height. Asymmetric error portion. In double membrane designs the asixymmetric error portion is assumed to be eliminated. 


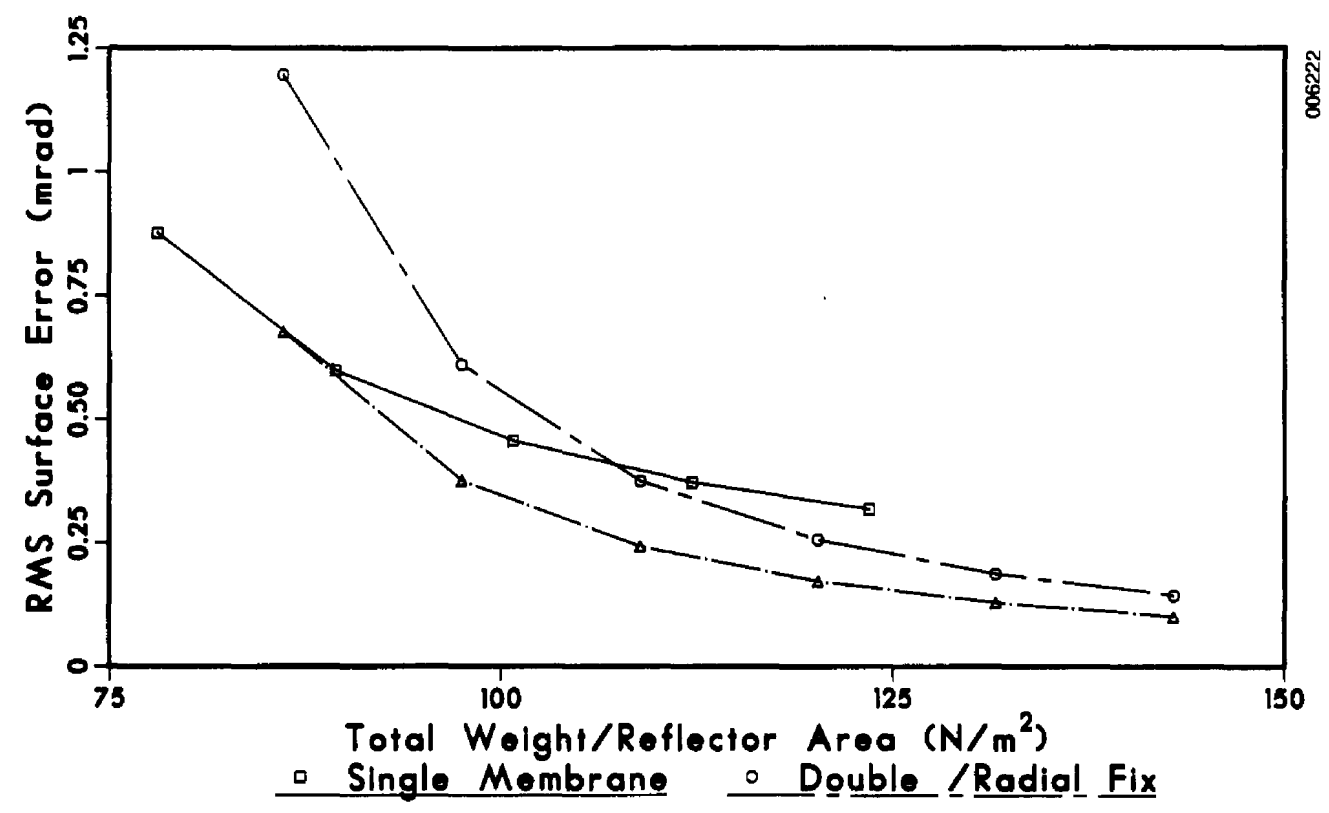

$\triangle$ Double /Radial \& Circum Fix

Figure B-10. RMS (Surface Averaged) Error as a Function of Total Module Weight per Unit of Reflector Area. Asymmetric error portion.

concept. We have established a reasonable bound on these errors of about 2 mrad rms total in addition to the surface errors corresponding to the glass metal concepts. This bound is based on assumed good design practice and could potentially be less than this level, especially for smaller radius designs. Of course, it is possible to do considerably worse than this, by inappropriate design of the unfocused module. For example, low initial tension levels and heavy (thick) membranes can push the error dramatically beyond the $2.0 \mathrm{mrad}$ level if care is not exercised.

It should be emphasized that neither the focused nor the unfocused designs are optimal, but are rather "typical" in that it is known that a number of improvements relative to the design considered here are possible. For instance, the frame thickness assumed in the analygis presented by Figures B-7 through $B-10$ is believed to be too thick for optimal conditions, and more than three supports may be desirable from a cost/performance perspective.

One final note of importance is that in designing an unfocused module, the process is more complex than merely taking a focused module and leaving off the controls and vacuuming subsystem. For instance, the desired operating tension would be considerably higher than typically assumed by SNLL for their controlled modules. Second, the lighter and thinner membranes of the unfocused module are also much more desirable in terms of performance considerations. In addition, it appears that different tension levels in the front and back membranes may be desirable (the higher tension being in the front membrane with the reflective surface on $i t)$. Further, different tension levels in the membranes need not mean unbalanced moments on the frame since the height above the frame torsional shear center might be varied to account for this. 


\section{APPENDIX C}

\section{PERFORMANCE OPTIMIZED CENTRAL RECEIVER \\ DESIGI PROCESS}

\section{C.1 INTRODUCTION}

This section describes the process used to generate the performance optimized system designs for the cases considered in the body of this report. The process makes use of a variety of computer codes, some of which are quite specific to a particular task, and others of which have much more general application. This process, and the results that were generated from it, represent an extension of earlier work reported in Delaquil and Anderson [11]. A flow diagram of the process described here is shown in Figure $C-1$.

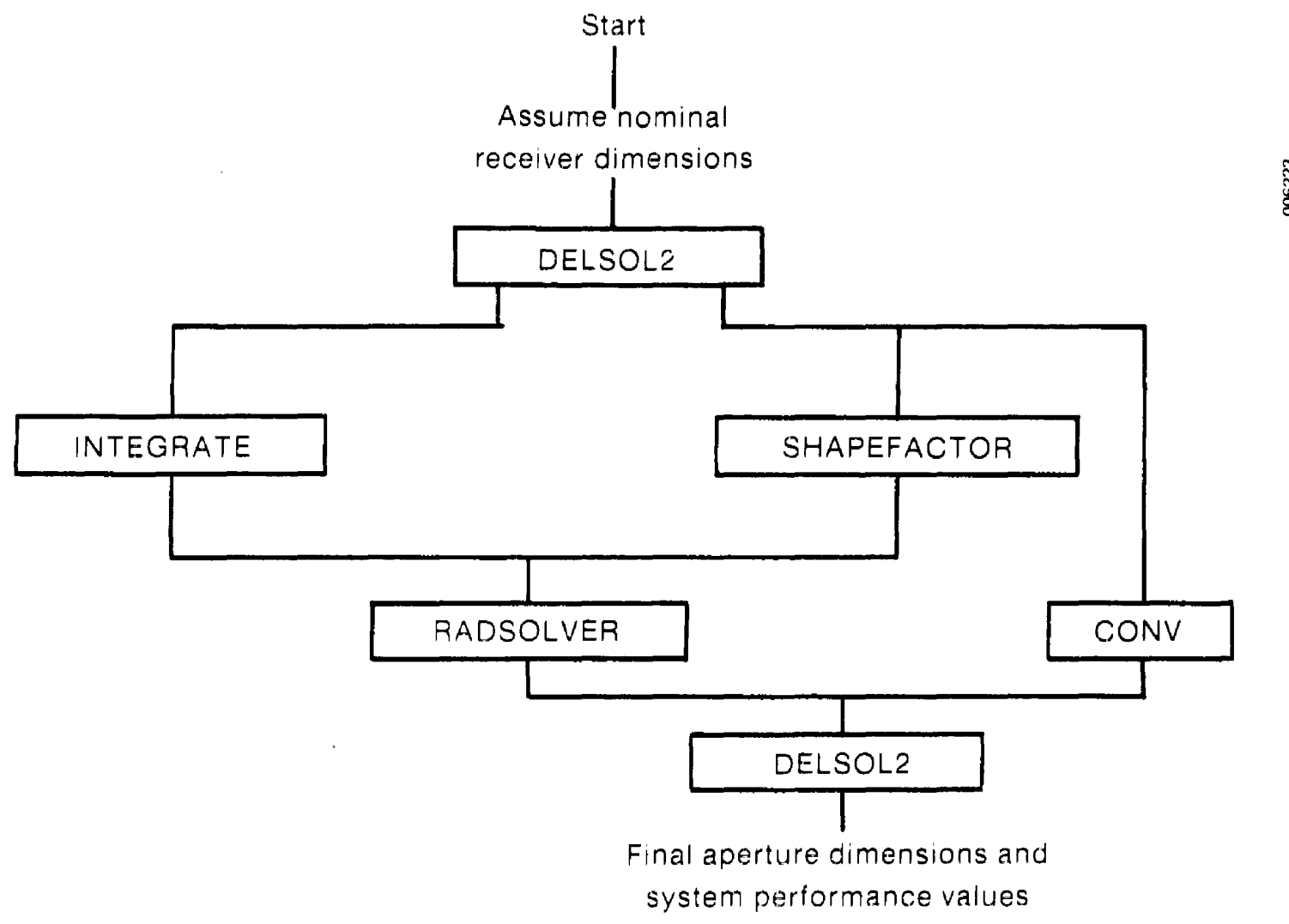

Figure C-1. Flowchart of Design Procedure 
The general process involves three stages:

1. Assuming some initial receiver characteristics, calculate the basic tower/field geometry and determine approximate receiver dimensions, using the DELSOL2 code [10].

2. For the tower/field geometry and rough receiver dimensions determined in the first stage, calculate both the radiative and convective losses from the receiver.

3. Finally, with the improved receiver losses calculated in the second stage, repeat the DELSOL2 analysis and perform the final optimization on the aperture dimensions, trading off spillage againgt the receiver thermal losses.

Although this process could be repeated several times, with each iteration resulting in a slightly improved estimate of the "true" optimum configuration, testing has shown that in practice very reasonable accuracies are achieved with a single pass.

The following paragraphs describe in more detail the steps taken in each of these stages.

\section{C.2 FIRST STAGE - TOWER/FIELD AND APPROXIMATE RECEIVER DIMENSIONS}

The first stage of the process involved the use of DELSOL2 exclusively. Because interest in this study was focused on system performance as a function of several parameters, the normal (economics-based) optimization scheme used in DELSOL2 had to be side stepped. Thus, the figure of merit used to define an optimum system was the annual efficiency instead of the busbar energy cost.

The firgt step was to chooge nominal starting values for the receiver dimensions and thermal losses, and a general range of tower heights and field sizes. The first parameter to be optimized was the tower height.

As illustrated in Figure C-2, the variation in system performance with tower height shows a very broad maximum. It was felt that choosing the tower height that produced the absolute maximum system performance was somewhat misleading, since nearly equal performance (within half of a percentage point) could frequently be achieved by a tower that was as much as $20 \%$ shorter.

Therefore, the criterion used for determining the tower height was the minimum value that produced performance values within about half of a percentage point of the maximum. Using this criterion produced very consistent results in terms of the tower-field geometry. The ratio of the tower height to total mirror area was nearly constant, and the rim angle to the farthest (north) heliostat was consistently around $15 \%$.

Once the tower height had been determined, the next step was to look at a range of cavity depths and aperture widths. For a given tower/field geometry, the cavity depth is determined completely by the flux limits on the absorber surface. The limits used in this study were $600 \mathrm{MW} / \mathrm{m}^{2}$ peak flux on the absorber. (This is near the upper limit of flux levels allowable on conventional super-alloy tubes with water/steam or molten-salt fluids.) 


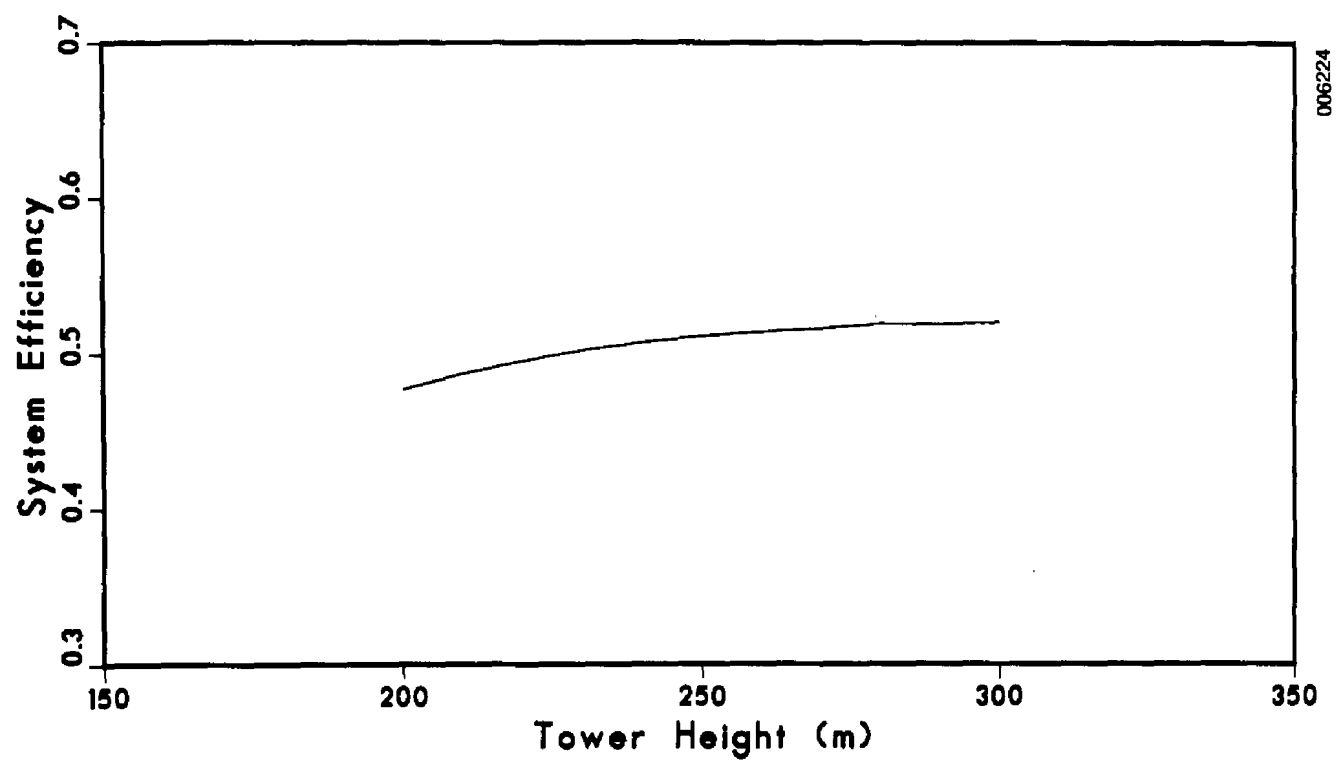

Figure C-2. Variation of System Performance with Tower Height

The aperture dimengions are determined by trading off spillage (the amount of radiation incident upon the aperture plane that does not pass through the aperture) against thermal (both radiative and convective) losses from the receiver. As the aperture dimensions increase for a given receiver, the spillage will decrease and the thermal losses will increase.

Obviously, the outcome of this tradeoff depends on a number of factors, including receiver parameters such as geometry and operating temperature, as well as field characteristics such as focusing and canting of the heliostats and the size and shape of the field (both of which influence the composite image).

DELSOL2 optimizes the aperture dimensions by scaling the receiver thermal losses directly with the aperture area. Since in this first stage of the design process only approximate values of the losses are available, the height and width of the aperture are not varied independently, but rather the ratio of the height to the width is held constant and the losses and spillage are calculated for a range of aperture sizes.

Once the approximate aperture dimensions have been determined, DELSOL2 has enough information to produce a "map" of the flux levels on the absorber wall. Then the receiver depth and height, the approximate aperture dimensions, and the absorber flux map can be passed to the second stage of the design process. 


\section{3 SECOND STAGE - RECEIVER LOSSES}

The second stage of the design process involves calculating the receiver loases for the cavity geometry and aperture dimensions that were determined in the first stage. This calculation takes place in two parts: the radiative losses (both reflected and emitted) are calculated, and then the convective losses are calculated.

An isometric view of the receiver used in this study is shown in Figure C-3a. To calculate the radiative losses, the interior of the receiver is divided into a number of zones, each of which acts as an independent surface in receiving and emitting radiation. Although most of the inactive surfaces in the receiver are modeled with a single zone per wall, the absorber surface was subdivided into a number (on the order of 20 to 30 ) of smaller zones, as shown in Figure $\mathrm{C}-3 \mathrm{~b}$, because of the variation in the solar flux levels on it.

The ultimate calculation of the radiative losses is done by a program called RADSOLVER [12], which was also written at Sandia National Laboratories, Livermore. RADSOLVER requires three sets of information to do its calculations:

1. The incoming solar flux profiles as calculated by DELSOL2

2. A description of the geometry of the receiver, in the form of a table of shape (or configuration) factors and the surface areas for each zone

3. The boundary conditions of the zones within the receiver (i.e., the temperature of--or heat flux through--that surface).

The values reported in the flux map output by DELSOL2 are local values calculated for a matrix of points on the absorber surface. For RADSOLVER to use them, this matrix of point values must be integrated and averaged over each of the zones in the radiative model. This is accomplished in a program that computes the double integral over each of the zone using a bicubic spline interpolant to "fit" the matrix of point values from DELSOL2. These integrated values are averaged over the zones, and then output from the program along with the total flux incident on the absorber.

The tables of shape factors and zone areas are output from a program named SHAPEFACTOR [13], which was originally developed at the University of Washington. It uses a geometric description of the vertices (corners) of each of the zones to assemble a model of the interior of the receiver and calculate how much of the radiation leaving a particular zone will strike each of the other zones (i.e., the extent to which each zone can "see" each of the other zones). It also calculates the areas of each of the zones.

In the terms used in this problem, the boundary condition information generally describes whether the zone in question is an active (heat-absorbing) surface or an inactive one. Since the inactive surfaces are assumed to be well insulated, with negligible conduction losses (since we are ignoring the interaction between radiation and convection), they are described to the model as adiabatic (zero net heat transfer). The active surfaces are, of course, 

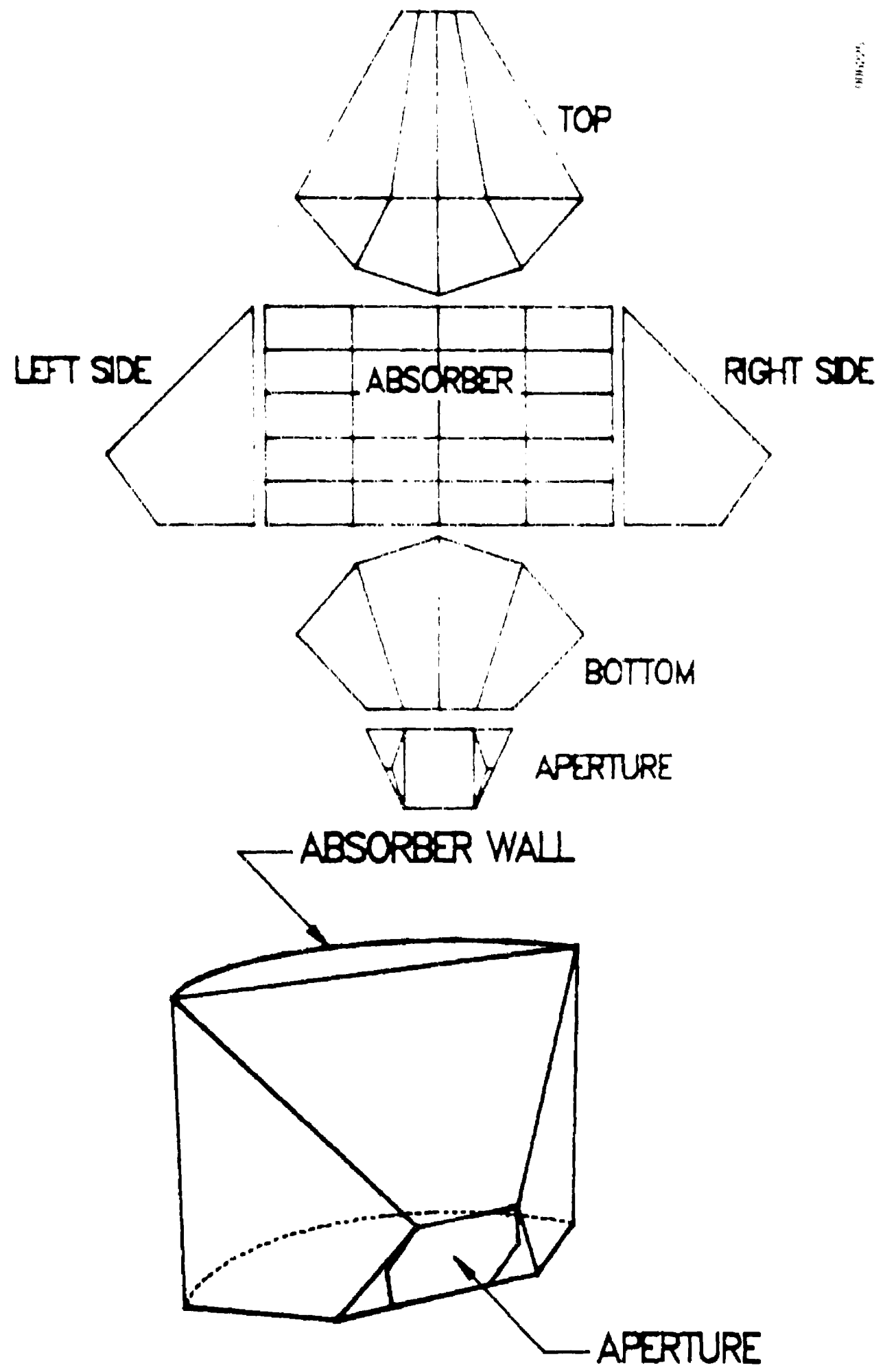

Figure C-3. (a) Expanded Layout and (b) Isometric View of the Baseline Receiver Cavity Configuration 
absorbing an as yet unknown amount of energy, so it is not possible to specify the net heat flux through them. We do know, however, what temperature we want them to be operating at, and this provides the requisite boundary condition.

The other type of information that could loosely be classified as a boundary condition and is required by the model is the hemispherical reflectivity of each of the zones. For the study reported here, the active surfaces were given a reflectivity of 0.1 , while the inactive surfaces were assigned a value of 0.4 .

Once these three pieces of information have been gathered, they form the input to RADSOLVER. Although RADSOLVER is capable of performing the radiative exchange calculations on a number of spectral bands, the single-band or graybody approximation was used. This is a common "first order" approximation, which significantly reduces the required amount of calculational effort. Since the situation being modeled was quite generic, the minor loss of accuracy associated with using this approximation was judged to be well within the uncertainty with which the multiband surface properties would be known.

The convective losses are calculated using a correlation proposed by Kraabel and Siebers [20] of Sandia National Laboratory. The correlation is:

$$
h=0.81\left(T_{w}-T_{a}\right)\left(A_{b o t} / A_{t o t}\right)^{0.63},
$$

where

$$
\begin{aligned}
h= & \text { the overall heat transfer coefficient based on the total } \\
& \text { interior of the cavity }\left(\mathrm{W} / \mathrm{m}^{2}{ }^{\circ} \mathrm{C}\right) \\
T_{w}= & \text { the interior wall temperature }\left({ }^{\circ} \mathrm{C}\right) \\
T_{a}= & \text { the ambient temperature }\left({ }^{\circ} \mathrm{C}\right) \\
A_{\text {bot }}= & \text { the interior area of the cavity below the top of the aperture } \\
& \text { (including the floor) } \\
A_{\text {tot }}= & \text { the total interior area of the cavity. }
\end{aligned}
$$

The wall temperatures in Kraabel's tests were nearly isothermal. In a real receiver, of course, there are significant variations in the wall temperatures between surfaces, especially between the active surfaces and the inactive surfaces. This situation is particularly acute for direct absorbtion receivers, where the absorbing fluid may be entering the top of the cavity $400^{\circ}-500^{\circ} \mathrm{C}$ colder than it leaves at the bottom. The approach taken here is to calculate an area-weighted average interior surface temperature based on the temperatures calculated in the radiative model. This area-weighted average is then used in Eq. C-1 to calculate the convective losses.

One drawback of the scheme used here for calculating the receiver losses is that the convective and radiative losses are calculated independently. Although these two mechanisms are not truly independent, this approximation is quite good as long as the receiver loss fractions are reasonably small. 
However, the procedure would lead to increasing overprediction of the losses at high loss fractions.*

\section{C.4 THIRD STAGE - APERTURE OPTIMIZATION AND PERFORMANCE ANALYSIS}

The third stage of the analysis again uses DELSOL2. In this stage, the aperture dimensions are optimized. As before, a range of aperture dimensions is tested, and the combination of width and height that produces the best annual system performance is chosen. This analysis is conducted with the other system parameters (e.g., field/tower geometry and cavity depth) that were determined in the earlier analysis. Obviously, if the newly optimized aperture dimensions are radically different from the originally assumed values, the second stage should be repeated to determine better loss values. However, it is usually possible to choose the original dimensions close enough to the final ones so that such iteration is not necessary.

Finally, once the aperture dimensions for optimum performance have been determined, a final DELSOL2 run is made to calculate a detailed summary of both the design point and the annual system performance.

*In order to correctly account for this interaction, it would be necessary to be able to predict the convective losses from each wall individually. Then the convective and radiative losses could be calculated iteratively, a procedure that would probably converge fairly quickly. Unfortunately, there is no well-verified method now available for predicting convective losses from individual surfaces in the complex flow situation found inside the receiver. Additionally, the error associated with this approximation for small loss fractions is probably no worse than the inaccuracies associated with a number of the parameters or models used in the analysis. For these reasons, the losses are calculated without including this interaction, and this fact is simply noted. 


\section{APPENDIX D}

PERFORMANCE OPTIMIZATION VERSUS COST/PERPORMANCE OPTIMIZATION

In the system analysis portion of this study, the system is designed solely on the basis of performance. The optimum design $\bar{D}$ is assumed to be the one that meets the preselected annual delivered energy (E) and temperature requirements with the highest efficiency; i.e., the design yielding the highest delivered energy per square meter of heliostat.

$$
\frac{E}{A_{C}(\bar{D})}=m_{p} x \frac{E}{A_{c}(D)} \text {, }
$$

where $A_{C}(D)$ is the field area for design $D$.

In reality, the optimum design is the design that minimizes system cost while still meeting the preselected delivered energy and temperature requirements. The system cost $\mathrm{C}_{s}$ comprises the costs of the individual components in the system.

$$
C_{g}=A_{C}(D) C_{H}+C_{T}(h)+C_{R}+C_{C}+C_{B O S}
$$

where

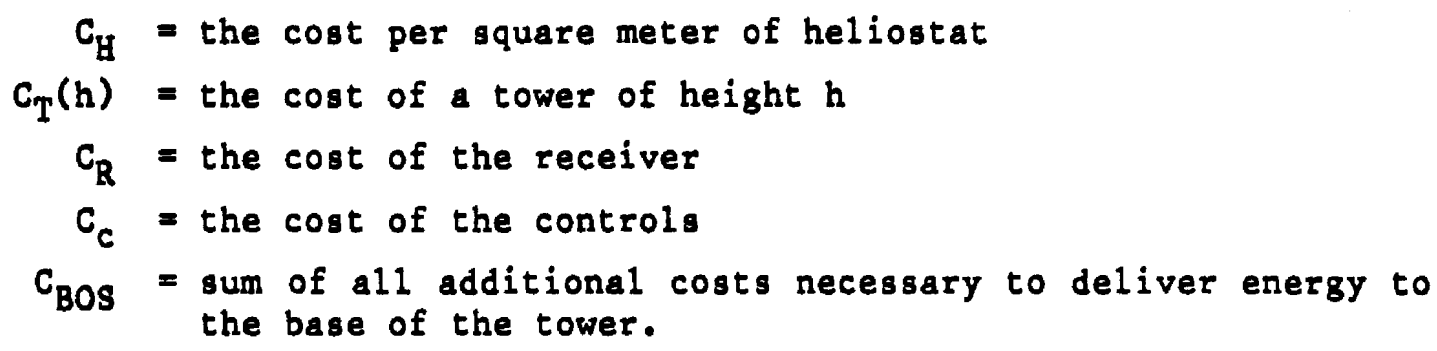

In selecting the optimal design three principal parameters are varied--the field area, tower height, and receiver aperture size. Since the cost of controls and balance of system do not vary appreciably with any of these three parameters, they need not be considered in a cost/performance optimization. Thus Eq. D-2 can be reduced to

$$
C_{s}(D)=A(D) C_{H}+C_{T}[h(D)]+C_{R}(D)+K^{\prime \prime},
$$

where $K^{\prime \prime}$ is a constant cost and all other costs are a function of the design D.

Current knowledge of the sensitivity of the cost of a single cavity receiver to changes in the aperture gize is extremely limited. Furthermore, our performance optimizations using DELSOL2 indicate that the optimum receiver configuration is ingensitive to the characteristics of the different types of heliostats that we examine in this study. Therefore in the cost/performance optimization the receiver cost can be considered to be a constant equal to the receiver cost in the base case configuration, and $\mathrm{Eq}$. D-3 reduces to

$$
C_{g}(D)=A_{C}(D) C_{H}+C_{T}[h(D)]+K^{\prime} \text {. }
$$


Earlier studies [21] have shown that tower cost $C_{T}$ is highly uncertain but sensitive to tower height. However, as shown in figure C-2 of Appendix $C$, system performance is highly insensitive to tower height. Therefore for all practical purposes the tower height can be assumed to be a constant, and Eq. D-4 reduces to

$$
C_{s}(D)=A_{C}(D) C_{H}+K \text {. }
$$

Thus our cost/performance optimization has been reduced to the following problem:

$$
\min C_{s}(D)=\min A_{C}(D) C_{H} \text {, }
$$

subject to the constraint that $E(D)=E$ where $E(D)$ is the annual energy delivered to the base of the tower by design $D$. But this is equivalent to maximizing the annual energy delivered per square meter of heliostat. Thus the optimal design $\bar{D}$ is such that

$$
\begin{array}{ccc}
E & E \\
A_{C}(\bar{D}) & D & A_{C}(D)
\end{array}
$$

and a cost/performance optimization yields the same optimal design and optimal system cost as the performance optimization performed in this study (compare Eqs. $D-1$ and $D-7)$.

To arrive at this conclusion concerning the essential equivalence of a performance optimization and a cost/performance optimization, we made some simplifying assumptions concerning the cost of receivers and the performance sensitivity to tower height. We intend to examine these assumptions in the future as more cost data become available. 


\section{APPEINDIX B}

\section{SPECULARITY AND COIE SIZE RELATIONS}

The analysis presented below provides a simple approximation for describing and relating specular reflectance, specular cone size and the total amount of energy reflected within that cone size.

As discussed by Pettit et al. $[7,22,23,24]$, an incoming collimated beam of light $F$ is reflected from a reasonably good solar reflector not as a simple perfectly specular beam but rather as scattered 1 ight about the nominal reflected ray defined as the specular direction $\vec{R}$ in a fairly narrow cone (see Figure E-1). Further, it is shown that the intensity of the reflected beam, measured with respect to the specular direction, is proportional to the product of the total solar averaged hemispherical reflectance*, $o_{s}$, and a geometric factor $G(\Psi)$, which is a function of the half cone angle, $\Psi$, measured from the specular direction. Their data also shows that the scatter of the reflected light about the specular direction can often be accurately represented by a normal or Gaussian probability distribution.

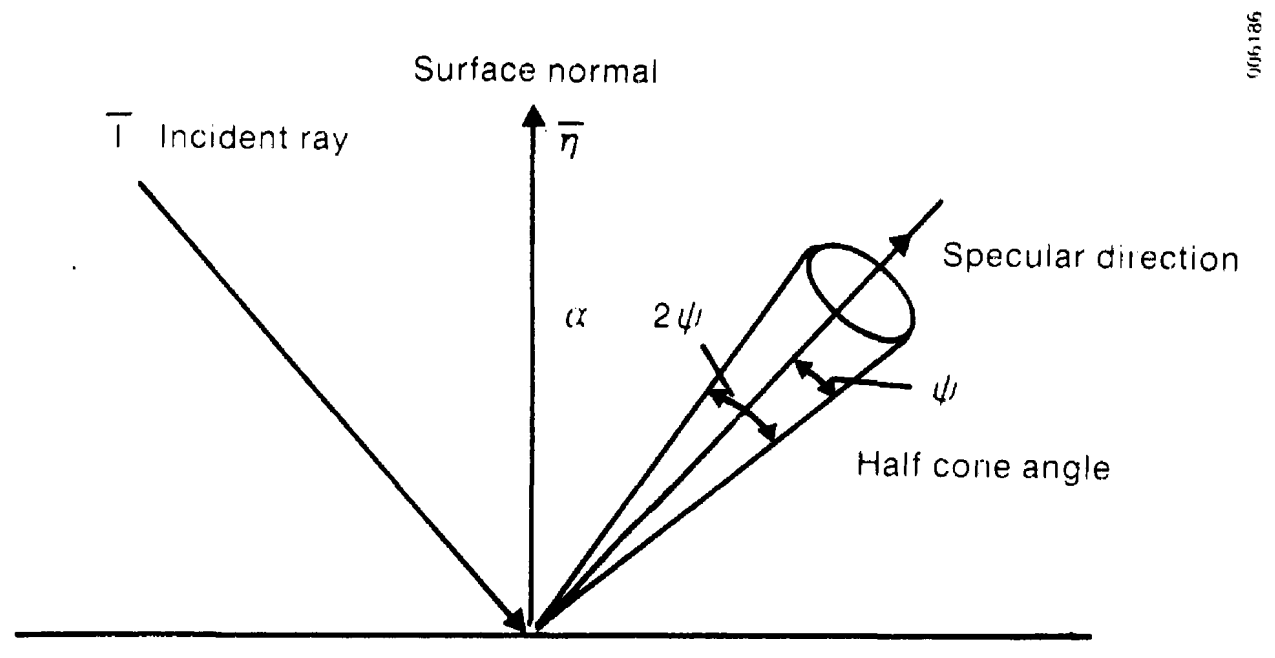

Figure E-1. Cone Containing Scattered Beam about the Specular Direction

*Averaged over the entire range of wave lengths in the solar spectrum. 
If we assume that the distribution is a simple circular Gaussian* distribution about the specular direction, then the fraction, ${ }^{0}(\Psi)$, of the incoming radiation that is reflected within a cone about the specular direction, having a half angle $\Psi$, is given by

$$
\rho_{T S}(\Psi)=\rho_{g} G(\Psi)
$$

$G(\Psi)$ is the cumulative distribution function corresponding to the circular Gaussian distribution and is defined by

$$
G(\Psi)=1-e^{-1 / 2\left(\frac{\psi}{\sigma_{\Psi}}\right)^{2}}=\frac{1}{2 \pi} \int_{0}^{2 \pi} \int_{0}^{\Psi} \frac{\phi}{\sigma_{\Psi}{ }^{2}} e^{-1 / 2\left(\frac{\phi}{\sigma_{\Psi}}\right)^{2}} \mathrm{d \phi dz},
$$

where $\sigma_{\psi}$ is the standard deviation of the half-cone angle $\Psi$. With the above definitions $G(\Psi)$ can be interpreted as the ratio of the total specular reflectivity corresponding to a given cone angle size to the total hemispherical reflectivity.

It is important to note that $\Psi$ is the half-cone angle whereas the data from tests are typically given for a full-cone angle a $(=2 \Psi)$. This difference in conventions has evolved since analytical models usually employ probability distributions in which the half-cone angle is the random variable, whereas many two-dimensional experiments measure the energy within the full-cone angle.

Figure E-2 shows the fraction of energy (G) within a given half-cone angle as a function of $\Psi / \sigma_{\psi}$. It is seen that for half-cone angles of $\Psi=\sigma_{y}, 2 \sigma_{y}$, and $3 \sigma_{\psi}$, the corresponding energies within the cone are about $40 \%, 90 \%$, and $99 \%$, respectively. We can use Figure E-2 to determine, for given values of $G(\Psi)$ and $\Psi$, the corresponding value of $\sigma_{\psi}$. Alternately the standard deviation of $\Psi$ can be determined by inverting $\mathrm{Eq}$. E-2 to give

$$
\sigma_{\psi}=\frac{y}{\sqrt{-2 \ln (1-G(\Psi))}}=\frac{\frac{a}{2}}{\sqrt{-2 \ln (1-G(\Psi))}} .
$$

*The uniform circular Gausian distribution assumes that distribution about the specular direction $\vec{R}$ is axisymmetric. Nonaxisymmetric situations can be considered if sufficient data are available [9].

**This form assumes that a single circular normal distribution is sufficient to describe the scattering. Pettit describes cases in which two such distributions are linearly superimposed. In most cases, however, a single distribution provides a very good approximation.

***This of course assumes that a single circular normal distribution adequately describes the scattering. 


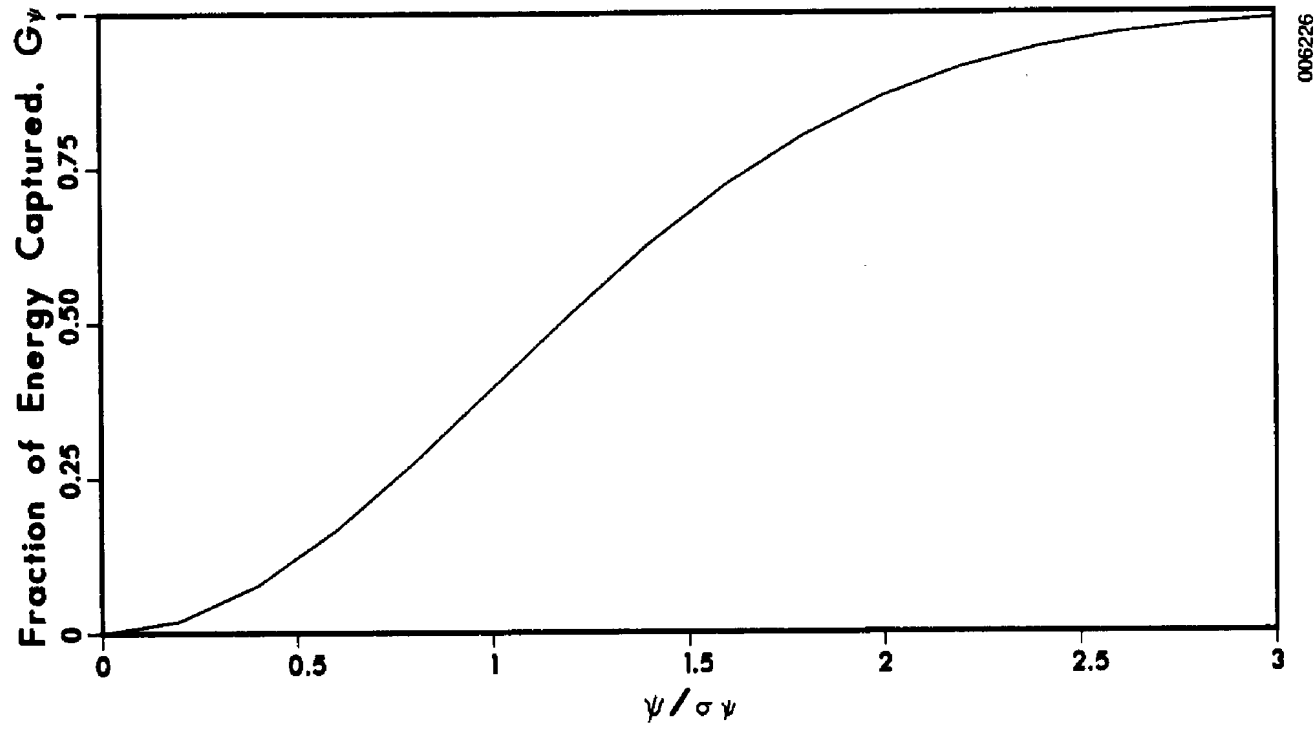

Rigure E-2. Fraction of Reflected Energy in a Given Cone Size

The form of Eqs. E-1 and E-2 can also be used to incorporate other beam broadening effects in addition to specularity in the calculation of the fraction, $\rho_{T S}(y)$, of the incoming radiation, which is reflected within a cone with half angle $\Psi$. Let $\theta_{\text {refl }}$ be the half-cone angle that includes not only specularity but also $n$ other beam broadening effects, $\theta_{k}(k=1,2,--, n)$

$$
\theta_{\text {ref } 1}=\Psi+\sum_{k=1}^{n} \theta_{k} .
$$

If these other effects can be assumed to be independent and circular normally distributed, then the distribution of $\theta$ fef 1 found by convolving the distributions of the different effects will afso be circular normally distributed. The standard deviation of $\theta_{\text {refl }}$ in a single dimension, $\sigma_{\text {refl }}$, is then $g$ iven by

$$
\sigma_{r e f 1}^{2}=\sigma_{\psi}^{2}+\sum_{k=1} \sigma_{k}^{2} \text {. }
$$

Thus by Eq. E-2,

and by Eq. E-1,

$$
G(\theta)=1-\exp \left[-1 / 2\left(\frac{\theta}{\sigma_{\text {ref } 1}}\right)^{2}\right],
$$

$$
\rho_{\mathrm{TS}}(\theta)=\rho_{\mathrm{S}} G(\theta)=\rho_{\mathrm{S}}\left\{1-\exp \left[-1 / 2\left(\frac{\theta}{\sigma_{\text {ref }}}\right)^{2}\right]\right\} .
$$




\section{APPENDIX E}

\section{RECEIVER CONFIGURATION}

One of the areas of central receiver design that seems the least developed is that of the cavity receiver configuration*. Although the effect of the overall configuration of the cavity on radiative losses is readily apparent, current evidence [19] indicates that the convective losses are also dependent on the configuration, particularly on the amount of hot interior surface area and the placement of the aperture.

The cavity configuration used to generate the results presented in the body of this report is shown in Figure $\mathrm{C}-3$ in Appendix $\mathrm{C}$. It was originally designed for a study of high-temperature systems at fairly high flux levels [11]. Although it performs quite well for this range of parameters, a major limitation of this deaign is that it can accommodate only a limited aperture width before the inactive side walls begin to face outward through the aperture instead of inward at the absorber.

Unfortunately, for some of the systems that used the larger sizes of unfocused stretched membrane heliostats at the small plant size, the optimum aperture dimensions were larger than could be accommodated by this original configuration. In these cases, the results presented in the body of the report were generated using the largest aperture possible with this configuration.

To establish how far off the optimum these results were, another configuration was briefly investigated. An isometric of this new configuration is shown in Figure F-1. This new design allows much larger aperture sizes, but has some disadvantages in terms of the amount of hot inactive surface area.

The results from this new configuration did show an improvement in performance for systems requiring large aperture areas. The results are compared in Figure F-2 for the $100-\mathrm{m}^{2}$ unfocused stretched membrane heliostats at the baseline surface errors $\left(\sigma_{\text {opt }}=3.46\right)$. The maximum difference is almost $10 \%$ at the lower temperature, while the difference nearly disappears at the highest temperature. It appears from this data that there is a crossover point somewhere; on one side of this point the original receiver configuration will perform better, while on the other side the new design will be better.

*In this discussion, the cavity configuration refers to the overall geometry, including the shape of the absorber, the shape and angle of inclination of the aperture, and the geometry of the inactive surfaces that connect the aperture to the absorber. The particular dimensions of a configuration, including the height and depth of the cavity and the aperture dimensions, are optimized for each case considered. See Appendix C for a discussion of this optimization process. 


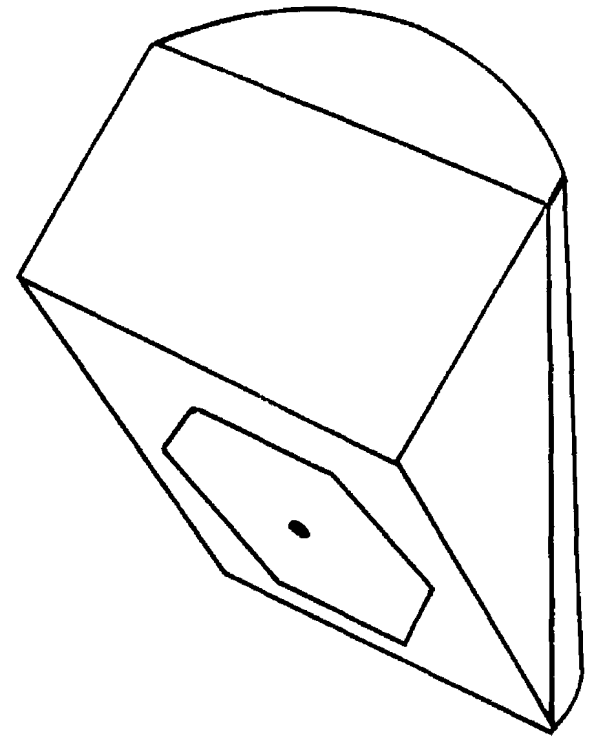

\&
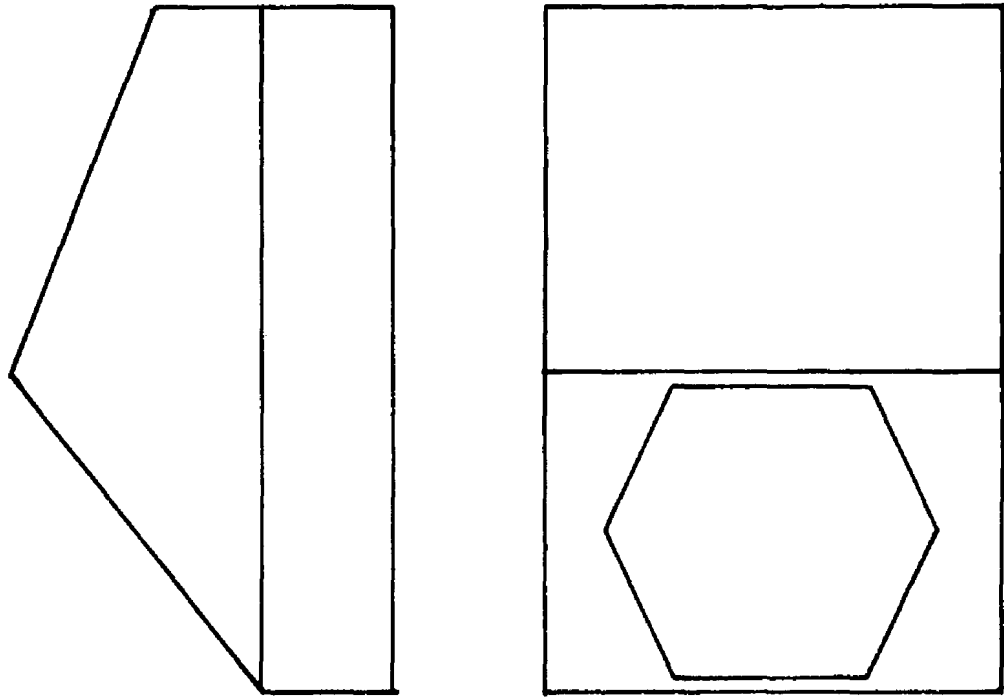

Figure B-1. Isometric View of Alternate Receiver Cavity Configuration Allowing Larger Aperture Size日

The original receiver configuration limits the optimum aperture size in only a few of the cases examined. Since this limitation does not seriously affect the overall results, for the sake of consistency all results presented in the body of this report are based on the original receiver configuration. 


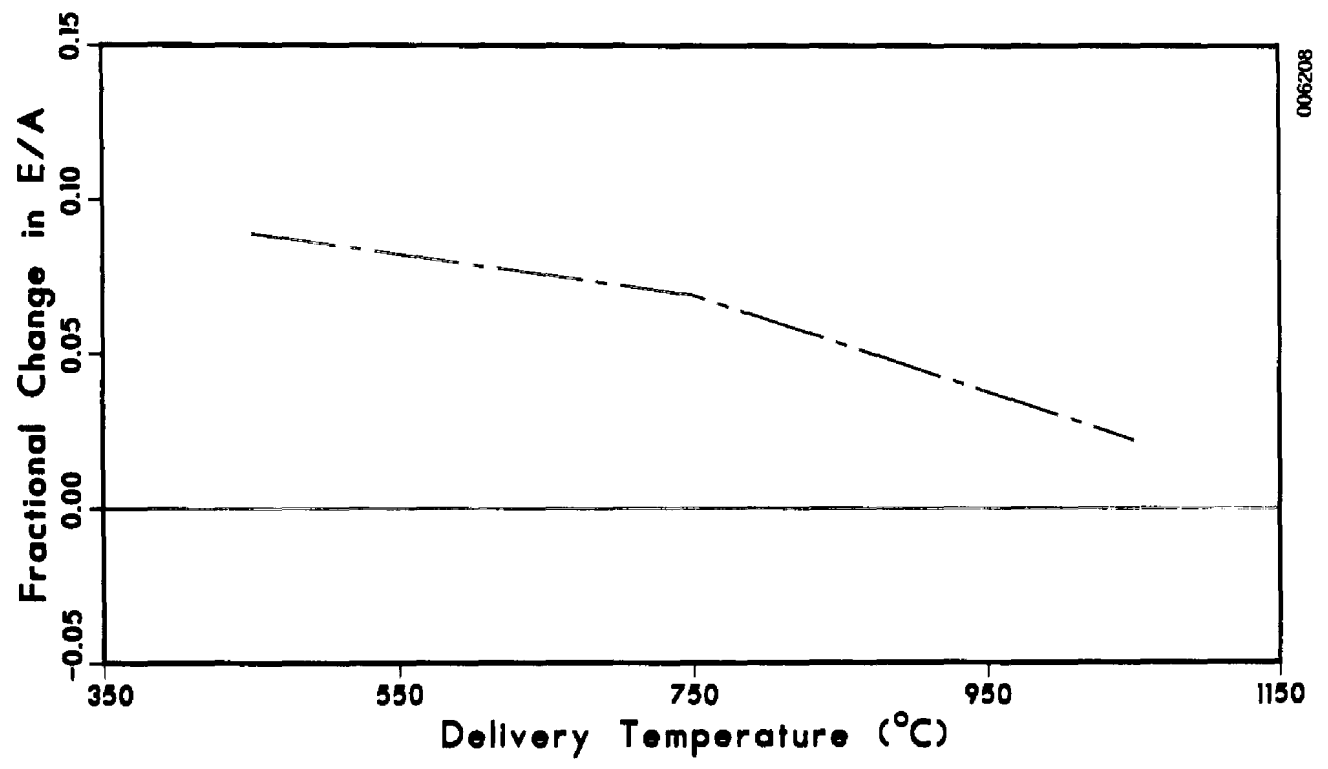

Figure F-2. Fractional Change in Delivered Energy as a Result of the Longer Aperture Sizes Possible $y^{i t h}$ the Reconfigured Cavity for a 75-MW $W_{\text {th }}$ Plant with $100-\mathrm{m}^{2}$ Unfocused Stretched Membrane Heliostats 


\section{DISTRIBUTION LIST}

Acurex Solar Corporation

485 Clyde Ave.

Mt. View, CA 94042

Mr. Don Duffy

Arizona Public Service Company P.0. Box 21666

Phoenix, AZ 85036

Mr. Eric Weber

BDM Corporation

1801 Randolph SE

Albuquerque, NM 87106

Battelle Pacific NW Laboratory P.0. Box 999

Richland, WA 99352

Dr. Ben Johnson

Mr. Tom Williams

Dr. Kevin Drost

Bechtel Corporation

P.O. Box 3965

San Francisco, CA 94119

Mr. Pascal DeLaquil

Black and Veatch Consulting

Engineers

1500 Meadow Lake Parkway

Kansas City, MO 64114

Dr. Charles Grosskreutz

Brumleve, Dr. Tom

Consultant

1512 N. Gate Road

Walnut Creek, CA 94598

Colorado State University

ERC

Ft. Collins, CO 80523

Dr. John Peterka

Dan-Ka Products, Inc.

1135 S. Jason Street

Denver, CO 80223

Mr. Daniel Sallis

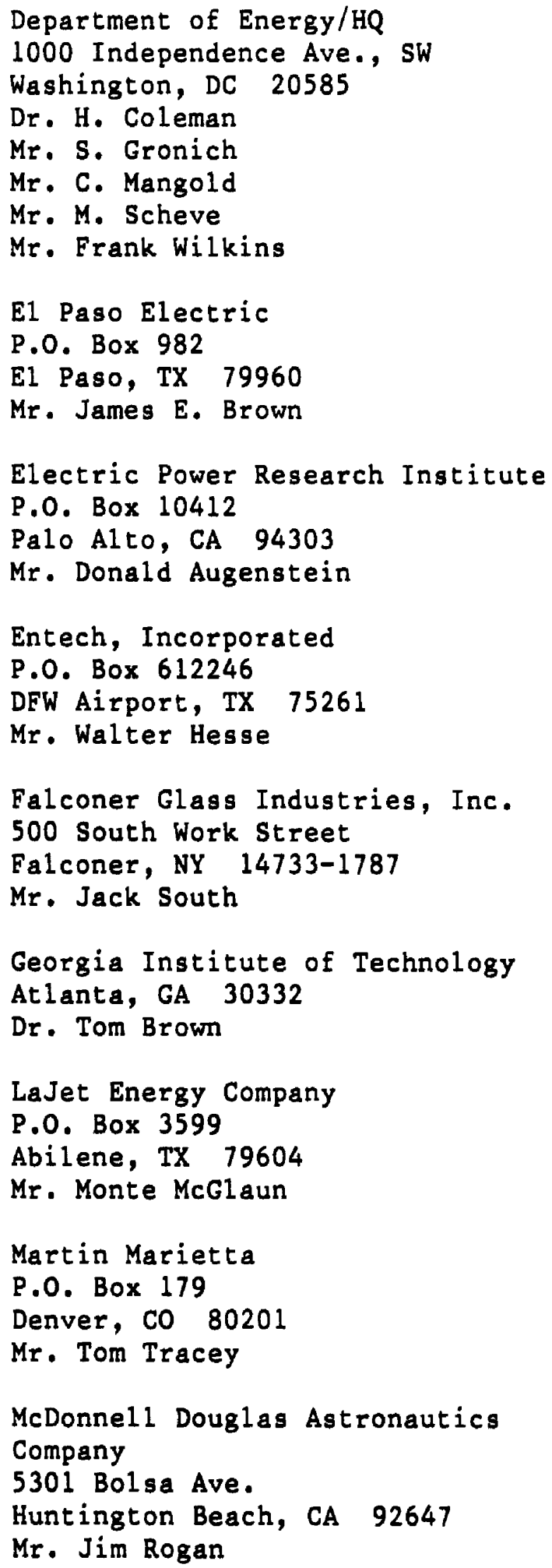


Meridian Corporation

5113 Leesburg Pike

Suite 700

Falls Church, VA 22041

Mr. Dinesh Kumar

Midwest Research Institute 425 Volker Blvd.

Kansas City, MO 64110

Mr. R. L. Martin

Mr. Jim Williamson

NASA Lewis Research Center

21000 Brookpark Road

Cleveland, $\mathrm{OH} \quad 44135$

Dr. Dennis Flood

NASA-Johnson Space Center

NASA Road One - EPS

Houston, TX 77058

Mr. William simon

New Mexico State University

Mechanical Engineering Dept.

P.0. Box 3548

Las Cruces, NM 88003

Dr. Ceorge Mulhollard

Pacific Gas and Electric Company

Mr. Gerry Braun

Mr. Joe Iannucci

3400 Crow Canyon Rd.

San Ramon, CA 94583

Rockwell International

Energy Systems Group

8900 De Soto Ave.

Canoga Park, CA 91304

Mr. Tom H. Springer

Sandia National Laboratories

Solar Department 8453

Livermore, CA 94550

Mr. J. C. Swearengen

Mr. A Skinrood

Mr. R. A. Rinne

Sandia National Laboratories Solar Energy Department 6220

P.0. Box 5800

Albuquerque, NM 87185

Mr. John Otts

Mr. James Leonard

Mr. Donald Schuler
Science Applications, Inc.

10401 Rosselle Street

San Diego, CA 92121

Dr. Barry Butler

Solar Energy Industries Association 1717 Massachusetts Ave. NW No. 503

Washington, DC 20036

Mr. Carlo LaPorta

Mr. David Goren

Mr. Hal Seilstad

Solar Energy Research Institute

1617 Cole Blvd.

Golden, CO 80401

Mr. B. P. Gupta

Dr. L. J. Shannon

Mr. Floyd Blake

Mr. John P. Thornton

Dr. Meir Carasso

Dr. David Johnson

Dr. Paul Schissel

Dr. Gordon Gross

Solar Kinetics, Inc. P.0. Box 47045

Dallas, TX 75247

Mr. Gus Hutchison

Southern California Edison

2244 Walnut Grove Ave.

Rosemead, CA 91770

Mr. Joe Reeves

University of Arizona

College of Engineering

Tucson, AZ 95721

Dr. Kumar Ramohalli

University of Chicago

Enrico Ferni Institute

5640 S. Ellis Ave.

Chicago, IL 60637

Dr. R. Wington

Dr. J. Gallagher

University of Houston

4800 Calhoun

106 SPA Building

Houston, TX 77004

$\mathrm{Dr}$. Alvin Hildebrandt

$\mathrm{Dr}$. Lorin Vant-Hull 
3M Corporation

3M Center Building, 207-1W-08

St. Paul, MN 55101

Mr. David Hill

Mr. Burton A. Benson 


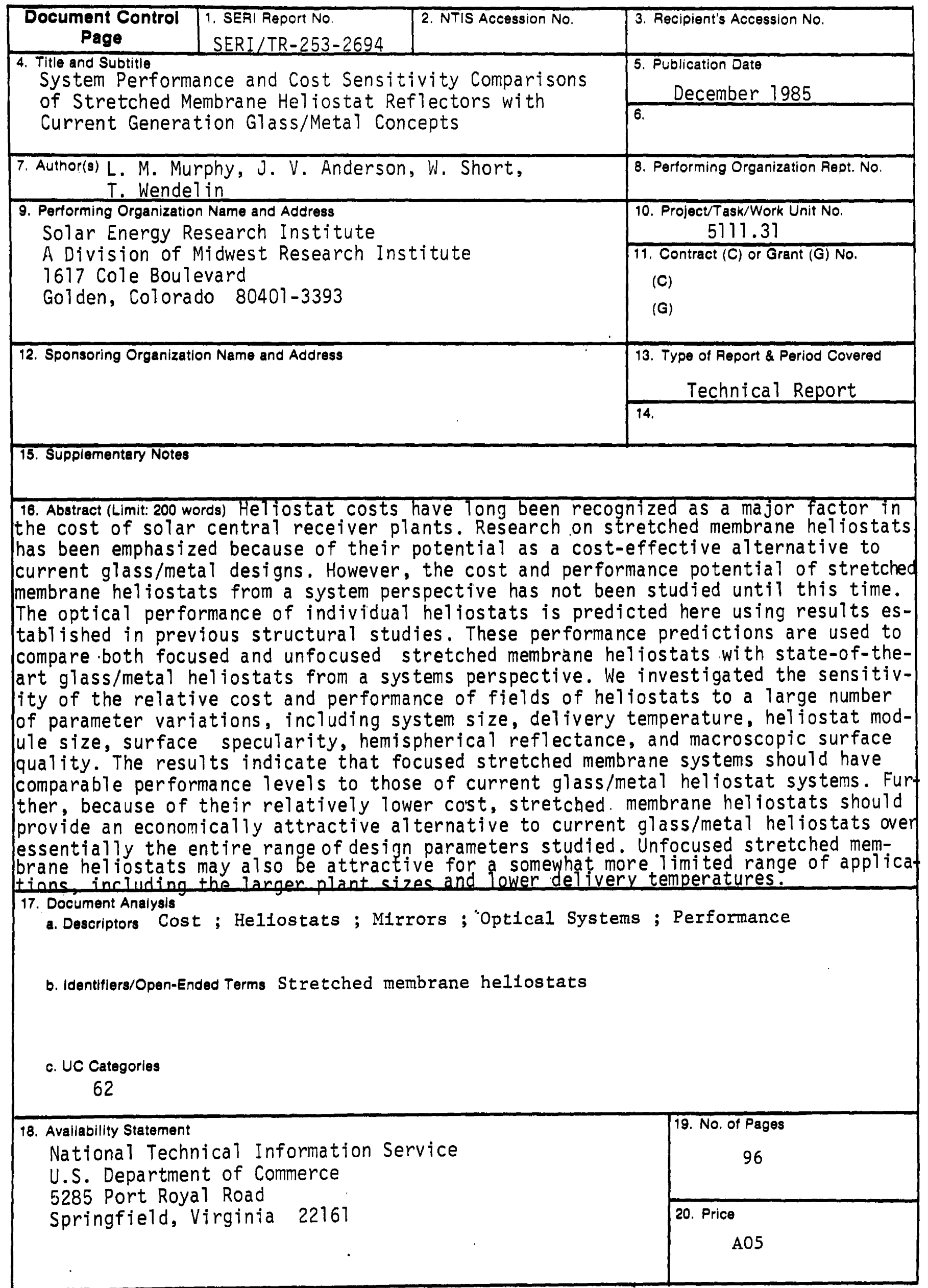

